\title{
Characterisation of the early endosomal SNARE complex
}

\section{PhD Thesis}

in partial fulfilment of the requirements for the degree "Doctor of Philosophy (PhD)" in the Molecular Biology Program at the Georg August University Göttingen, Faculty of Biology

\author{
submitted by \\ Daniel Zwilling \\ born in \\ Frankfurt/Main, Germany
}

2005 


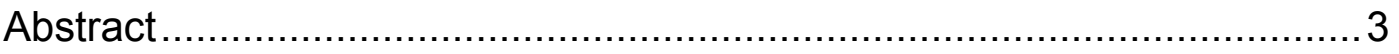

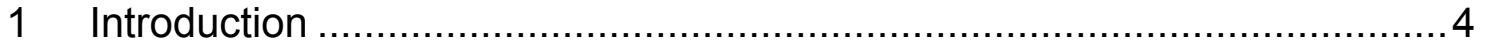

1.1 Intracellular Protein Transport and SNAREs ….......................... 4

1.1.1 SNAREs as Mediators of Membrane Fusion ................................... 5

1.1.2 Endocytosis, Homotypic Fusion of Early Endosome and the

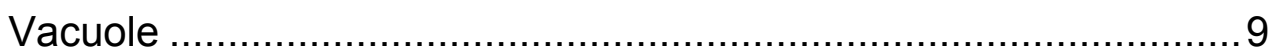

1.2 Candidates for the Early Endosomal SNARE Complex.................16

1.3 Liposomal Fusion, Compartmental Specificity and Topological

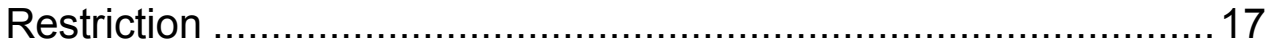

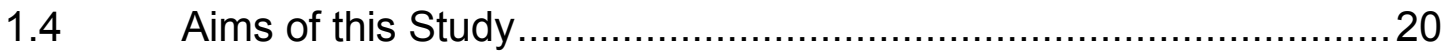

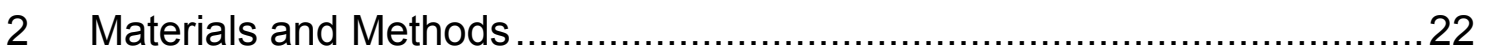

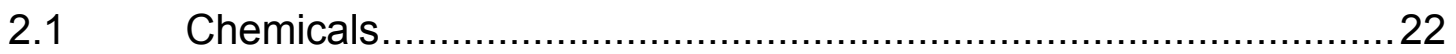

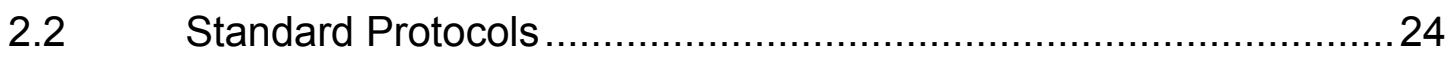

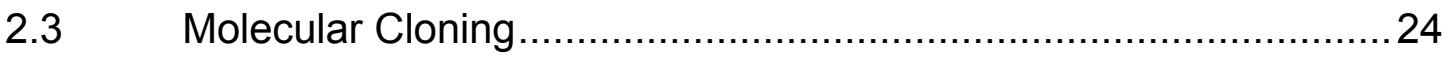

2.4 Expression and Purification of Recombinant Proteins ...................26

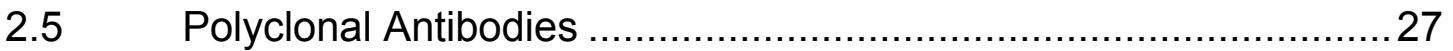

2.6 Assembly of the Early Endosomal SNARE Complex ...................28

2.7 Dissociation of the SNARE Complex by NSF ..............................28

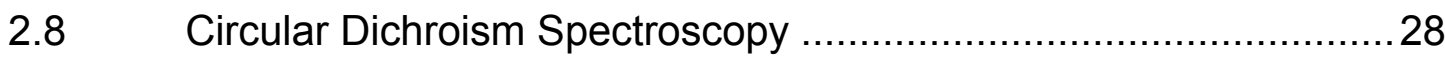

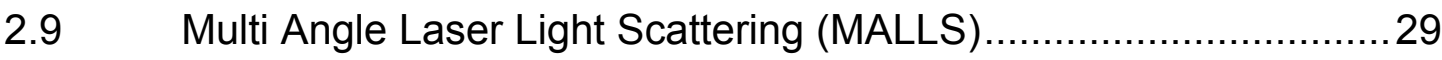

2.10 Crystallisation, Diffraction Data Collection, Structure Solution and

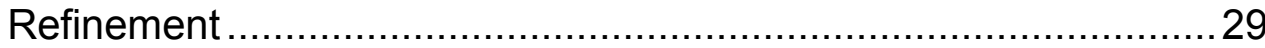

2.11 Preparation of Lipid Micelles …………………........................ 30

2.12 Determination of Orientation of SNAREs in Liposomes .................31

2.13 Liposomal Fusion Assays - Fluorescence Spectrophotometry ........32

2.14 Determination of $\mathrm{IC}_{50}$ Values in Inhibition Experiments ...................33

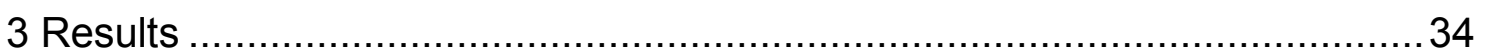

3.1 Biochemical Characterisation of the Early Endosomal SNARE

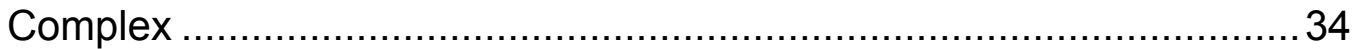

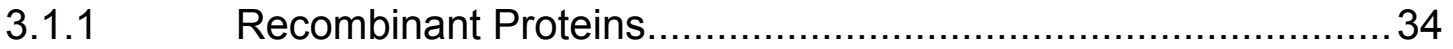

3.1.2 Characterisation of the Generated Antibodies ...........................37

3.1.3 Characterisation of the Function of the Recombinant SNAREs and the IgGs in the Early Endosomal Fusion Assay ...................38

3.1.4 Optimisation of the Assembly Conditions..................................45 
3.1.5 Assembly and Purification of the New SNARE Complex. .48

3.1.6 Molecular Mass Determination using Multi Angle Laser Light Scattering 51

3.1.7 The Early Endosomal SNARE Complex Exhibits

Unfolding-Refolding Hysteresis as Determined by

CD Spectroscopy. 52

3.1.8 Disassembly of the Complex Using NSF and $\alpha$-SNAP .............54

3.2 Crystal Structure of the Early Endosomal SNARE Complex ..........56

3.2.1 Overall Structure of the Complex ........................................5 56

3.2.2 Structure of the Individual Layers ......................................5

3.2.3 Intra- and Intermolecular Surface Interactions ......................59

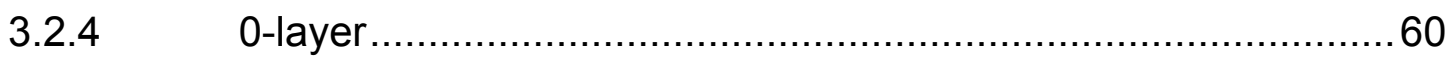

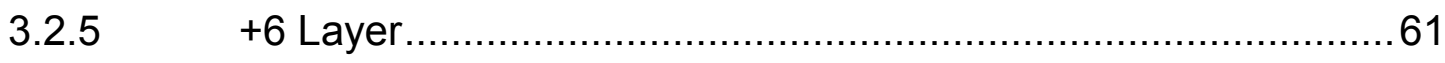

3.2.6 Interacting Residues are Conserved in Different Positions in the Respective SNAREs .............................................6 62

3.3 Characterisation of SNARE Mediated Liposomal Fusion ..............6 65

3.3.1 Fusion of the Proteoliposomes Containing the Early Endosomal SNARES 65

3.3.2 Inhibition of Liposomal Fusion with Soluble R-SNARE Fragments. 68

3.3.3 Different R-SNARE Liposomes are Able to Fuse with the QSNARE Liposomes 72

3.3.4.1 Topology-Dependent Fusion Investigated with the Dequenching

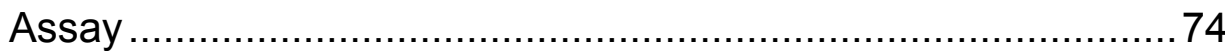

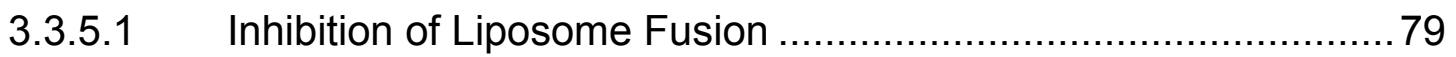

3.3.6 Liposomal Fusion Observed by FRET Pairing of Labelled Lipids83

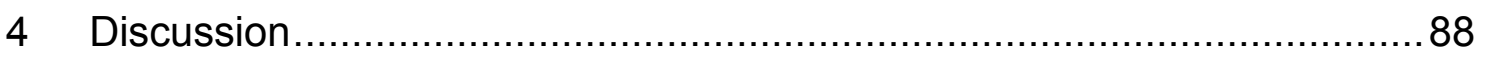

4.1 The Role of Syntaxin 6, Syntaxin 13, Syntaxin 16, Vti1a and VAMP4 in Early Endosomal Homotypic Fusion ............................................. 88

4.1.1 Subcellular Organisation of the Early Endosomal SNAREs........88

4.1.2 Function of the Early Endosomal SNAREs in fusion of PC12 early endosomes 89

4.2 Biochemical and Structural Features of the Early Endosomal SNARE Complex .90 
4.2.1 Biochemical and Biophysical Features ...................................90

4.2.2 Structural Features of the Early Endosomal SNARE Complex...91

4.3 Topological Restriction of the Early Endosomal SNARE Complex in

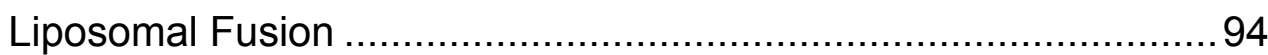

4.4 Specificity of the Early Endosomal SNAREs in Vitro and

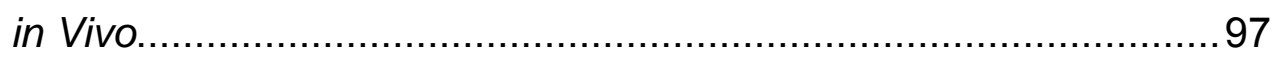

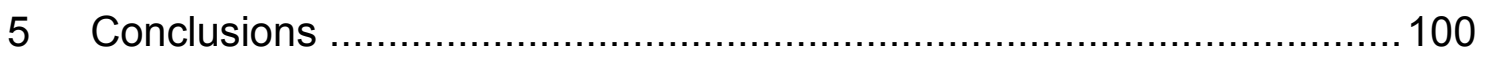

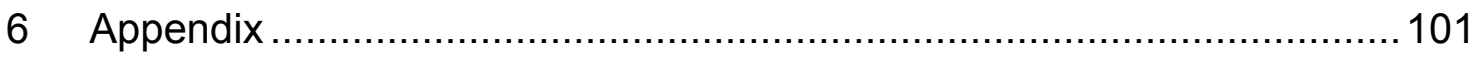

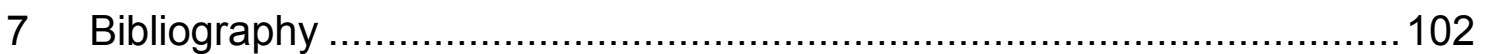

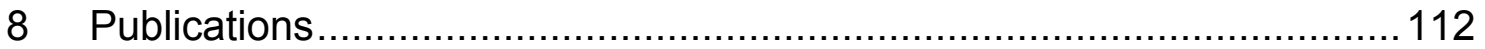

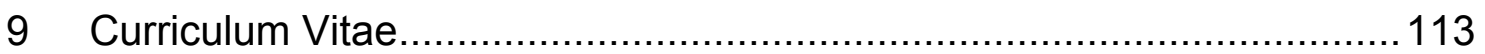

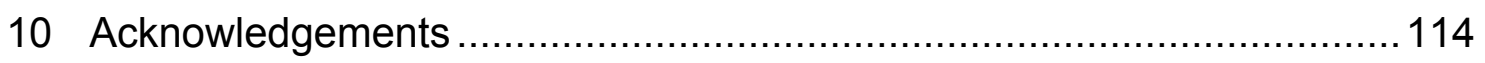

\section{Figures}

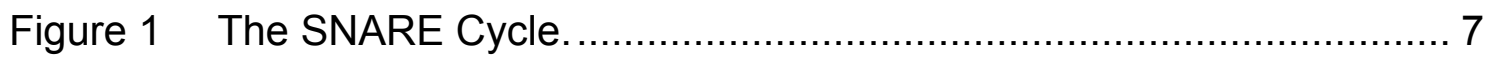

Figure 2 Intracellular Trafficking Routes. .............................................. 11

Figure 3 Trypsin Digest of Liposomes to Determine Orientation of SNARES.

Figure 4 Schematic Diagram of the Different SNARE Constructs............... 34

Figure 5 SDS-PAGE Analysis of the Five Recombinant SNARE Fragments.

Figure 6 Antisera against Syntaxin 6, Syntaxin 13, Syntaxin 16, VAMP4 and Vti1a Show Specific Signals on NRK or PC12 Cell Blots. 38

Figure 7 Inhibition of Early Endosomal Fusion using $F_{a b}-$ fragments Against Different Proteins.

Figure 8 Effects of Soluble Q- and R-SNAREs on Early Endosomal Fusion. 44

Figure 9 Analysis of Different Assembly Conditions using CD

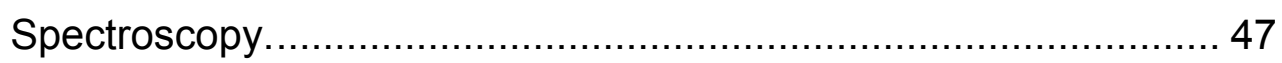

Figure 10 Purification of the Early Endosomal SNARE Complex. ................ 49

Figure 11 Analysis of the Fractions 7-9 with SDS-PAGE and Coomassie Blue Staining. 50

Figure 12 Determination of the Molecular Mass of the Early Endosomal SNARE Complex. 
Figure 13 Unfolding and Refolding Transitions of the Early Endosomal SNARE Complex Exhibit Hysteresis.

Figure 14 The Early Endosomal Complex is Dissociated by NSF and a-SNAP. 55

Figure 15 Overall Structure of the Early Endosomal SNARE Complex. 56

Figure 16 Structure of the Early Endosomal Complex is Highly Similar to the Neuronal Complex. ............................................. 57

Figure 17 Three SNARE Complexes in Sequence Comparison. 58

Figure 18 0-layer of the Early Endosomal Complex and Additional Ionic Interactions with Side Chains.

Figure 19 Layer +6 of the Early Endosomal SNARE Complex. 62

Figure 20 Intermolecular Stabilisation between Sx13 and Vti1a via Alternative Interactions. 63

Figure 21 Surface Interaction of the $Q_{b}$ - with the $Q_{c}$-chain. 65

Figure 22 Principle of the Dequenching Assay. 67

Figure 23 Example graph for the reaction VAMP4 + sx6-sx13-vti1a. 68

Figure 24 Dose-dependent Inhibition of Liposome Fusion by Increasing Amounts of Different Soluble R-SNAREs 70

Figure 25 Inhibition Experiments can be Evaluated by Plotting Inhibition vs. Concentration of Soluble Protein and Fitting the Trace with a Sigmoidal Function.

Figure 26 Different R-SNARE Liposomes Fuse with the Q-SNARE Liposomes Containing Sx6, Sx13 and Vti1a.

Figure 27 Fusion of Different R-SNARE Liposomes with Q-SNARE Liposomes Containing Sx6, Sx13 and Vti1a.

Figure 28 SDS-PAGE of all 14 Different Liposome Populations that Contained the $1: 3$ or 2:2 Topological Combinations.. 75

Figure 29 Liposomes Containing all Possible Non-Redundant Topological 1:3 or 2:2 Combinations of SNAREs. 76

Figure 30 Evaluation of the Topological Combinations in Liposomal Fusion using the Dequenching Assay.

Figure 31 Fusion Cannot be Inhibited in All Cases by Adding Soluble SNARE Fragments. 
Figure 32 'One-sided' Trypsin Digest of Liposomes Inhibits Fusion Potently 82

Figure 33 Bar-diagram of NBD/Rho Dequenching Signals of all 7 Topological Combinations, Trypsinated Liposomes and Negative Controls. .... 83

Figure 34 Principle of the Lipid FRET assay ............................................ 84

Figure 35 Fusion of Liposomes Monitored by Flourescently Labelled PE Lipids that Constitute a FRET Pair....................................................... 85

Figure 36 Bar Diagram of the Seven Topology Reactions Using the FRET

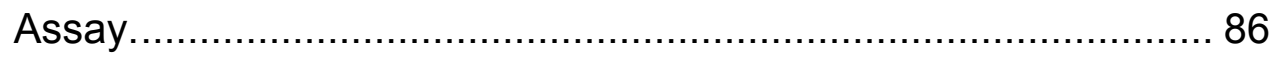

Figure 37 Possible Interactions Between Vti1 and Syntaxin 6 and Syntaxin 8 in Drosophila. 92

\section{Tables}

Table 1 SNAREs Involved in Different Fusion Steps in Mammals and Yeast..

Table 2 Overview of the Protein Constructs of Syntaxin 6, Syntaxin 13, Vti1a and VAMP4..

Table 3 Intra- and Intermolecular Interactions in the Early Endosomal SNARE Complex.

Table 4 Calculated IC 50 Values for the Three R-SNAREs VAMP4, Endobrevin and Synaptobrevin..

Table 5 Summary of Fusion Effiencies of Topological Combinations. 87

Table 6 Crystallographic Data 101 
Abbreviations and symbols

\begin{tabular}{|c|c|}
\hline$\AA$ & Angstrom \\
\hline aa & Amino acid \\
\hline APS & Ammonium persulfate \\
\hline ATP & Adenosine triphosphate \\
\hline ATPase & ATP hydrolysing enzyme \\
\hline a.u. & Arbitrary units \\
\hline $\mathrm{bp}$ & Base pairs \\
\hline BSA & Bovine serum albumine \\
\hline CCV & Clathrin coated vesicle \\
\hline CMC & Critical micelle concentration \\
\hline CNS & Crystallography and NMR system \\
\hline $\mathrm{dH}_{2} \mathrm{O} / \mathrm{ddH}_{2} \mathrm{O}$ & Distilled /double distilled water \\
\hline DTT & Dithiothreitol \\
\hline DNA & Deoxyribonucleic acid \\
\hline E.coli & Escherichia coli \\
\hline eb & Endobrevin \\
\hline EDTA & Ethylendiaminetetraacetic acid \\
\hline FFF & Free flow fractionation \\
\hline FRET & Fluorescence resonance energy transfer \\
\hline GSH & L-Glutathione reduced \\
\hline GST & Glutathion-S-transferase \\
\hline GTP & Guanosine triphosphate \\
\hline GTPase & GTP hydrolysing enzyme \\
\hline $\lg G$ & Immunoglobulin G \\
\hline IPTG & Isopropyl- $\beta$-D-thiogalactoside \\
\hline $\mathrm{h}$ & Hour(s) \\
\hline $\mathrm{kDa}$ & Kilo Dalton \\
\hline LB & Luria Bertani medium \\
\hline $\mathrm{mS}$ & Milli Siemens \\
\hline MALLS & Multi angle laser light scattering \\
\hline MWCO & Molecular weight cut off \\
\hline NBD & $\begin{array}{l}\text { 1,2-Dioleoyl-sn-Glycero-3-Phosphoethanolamine-N-(7- } \\
\text { nitro-2-1,3-benzoxadiazol-4-yl) (Ammonium Salt) }\end{array}$ \\
\hline NEM & $\mathrm{N}$-ethyl-maleimide \\
\hline Ni-NTA & Nickel-nitrilo-triacetate \\
\hline NSF & $\mathrm{N}$-ethylmaleimide-sensitive factor \\
\hline PAGE & Polyacrylamide gel electrophoresis \\
\hline PBS & Phosphate buffered saline \\
\hline PCR & Polymerase chain reaction \\
\hline PMSF & Phenymethylsulfonylflouride \\
\hline $\mathrm{pH}$ & Negative logarithm of $\mathrm{H}+$ concentration \\
\hline rpm & Revolutions per minute \\
\hline RT & Room temperature \\
\hline s & Second(s) \\
\hline $\mathrm{S}$ & Svedberg \\
\hline $\mathrm{Sb}$ & Synaptobrevin \\
\hline SDS & Sodium dodecyl sulfate \\
\hline
\end{tabular}




$\begin{array}{ll}\text { SEM } & \text { Standard error of the mean } \\ \text { SNAP } & \text { Soluble-NSF-attachment protein } \\ \text { SNAP-25 } & \text { Synaptosome associated protein of } 25 \mathrm{kDa} \\ \text { SNARE } & \text { SNAP-receptor } \\ \text { Sx6 } & \text { Syntaxin } 6 \\ \text { Sx13 } & \text { Syntaxin } 13 \\ \text { Sx16 } & \text { Syntaxin } 16 \\ \text { TB } & \text { Terrific broth } \\ \text { TEMED } & \text { N,N,N',N'-Tetramethylethylene diamine } \\ \text { Tris } & \text { Tris(hydroxymethyl)-aminomethane } \\ \text { vti1a/b } & \text { Vesicle transport through interactions with t-SNAREs 1a/b } \\ \text { V/v } & \text { Volume/volume } \\ \text { VAMP4 } & \text { Vesicle associated membrane protein } 4 \\ \text { W/v } & \text { Weight/volume }\end{array}$




\section{Abstract}

SNARE proteins are crucial factors in membrane fusion. These membrane proteins sit in opposite lipid bilayers and assemble to four-helix coiled-coils consisting of four SNARE helices: $Q a, Q b, Q c$ and R, thereby fusing the membranes. Syntaxin 13, vti1a, syntaxin 6 and VAMP4 were identified as the cognate SNAREs that mediate early endosomal fusion.

The core SNARE complex was purified, characterised using biophysical methods, and the crystal structure was solved with a resolution of $2.7 \AA$. The complex shows high similarity to the neuronal and the late endosomal complexes in terms of thermostability, biochemical behaviour and structure. Proteoliposomes containing these SNAREs fuse faster than proteoliposomes with the neuronal SNAREs and fusion can be inhibited in a dose dependent manner using soluble fragments of the cognate R- and Q-SNAREs, as well as the non-cognate R-SNAREs synaptobrevin and endobrevin. Furthermore, liposomes containing these non-cognate R-SNAREs fuse with the early endosomal Q-SNARE-liposomes, thus exhibiting a promiscuous behaviour.

This was not observed in a cell free, microscope-based, fusion assay, where soluble fragments of the proteins showed strong and specific inhibition of homotypic fusion of early endosomes from PC12 cells. 


\section{Introduction}

\subsection{Intracellular Protein Transport and SNAREs}

Eukaryotic cells contain a variety of membrane enclosed organelles that communicate with each other, i.e. they exchange proteins and lipids via directed trafficking. This membrane trafficking is a basic requirement in order to maintain the identity of the different cell compartments and the inner organisation of the cell. For instance, proteins destined for secretion are synthesised at the endoplasmic reticulum (ER), travel through the cis-, medial- and trans- Golgi compartment, before being further sorted and transported via vesicle transport to the plasma membrane where excretion occurs (Palade, 1975).

Each organelle has a defined localisation and function that demands a specific membrane composition of lipids and proteins. During trafficking a carrier vesicle pinches off from the donor compartment, is transported to and finally fuses with its acceptor compartment. A prerequisite for correct targeting is for the donor and acceptor membranes to recognise each other (tethering and docking) before the lipid bilayers fuse. All these processes are mediated by specific soluble and membrane resident proteins and are subject to high degrees of regulation.

An overwhelming body of evidence suggests that membrane fusion events in eukaryotic cells are mediated and regulated by specialised protein families that are conserved from yeast to humans (Bock et al., 2001). Membrane fusion is thought to be regulated by factors that belong to the super families of soluble NSF attachment protein receptors (SNAREs), Rab/ypt- and SM-proteins (Guo et al., 2000; Jahn et al., 2003; Pfeffer, 1999) and SNAREs represent the key players in membrane fusion. An exception is the fusion of mitochondria and peroxysomes which is mediated by unknown factors (Hermann et al., 1998; Sesaki and Jensen, 2001; Titorenko and Rachubinski, 2000). Before fusion, protein complexes are assembled on acceptor and donor membranes and these multimeric complexes mediate their adhesion. Rab proteins are key factors in membrane tethering (Zerial and McBride, 2001). These GTPases cycle between an active GTP-bound (membrane associated) and an inactive 
GDP-bound state (soluble). For Rab proteins to have an effect they must be in a membrane associated GTP-bound form (Takai et al., 2001). Rab/ypt GTPases are thought to be molecular switches that recruit tethering and fusion factors to the appropriate membrane. Rabs are regulated by so-called Rab-effectors that control GTP hydrolysis, GDP-GTP exchange and membrane association (Zerial and McBride, 2001).

After tethering, SNARE proteins and SM (sec1/munc18)-proteins mediate and control the fusion of lipid bilayers. SM -proteins are regulatory proteins that bind syntaxin like SNAREs (Jahn and Sudhof, 1999). Syntaxins exhibit a regulatory amino-terminal domain that can bind intramolecularly the SNARE motif resulting in an equilibrium of open and closed conformation (Dulubova et al., 1999; Fiebig et al., 1999; Munson et al., 2000). SM-proteins are able to interact with SNAREs in different ways. Their role in regulation of SNAREs is not fully understood. Over-expression of Munc-18 for example can result in both inhibition and potentiation of neurotransmitter release (Voets et al., 2001; Wu et al., 1998).

\subsubsection{SNAREs as Mediators of Membrane Fusion}

The super family of SNAREs comprises 24 members in yeast and more than 35 members in mammals (Bock et al., 2001). Initial experiments identified a soluble cytosolic protein called NEM sensitive factor (NSF) (Block et al., 1988). Factors that interacted with NSF were also isolated, so-called SNAPs (soluble NSF attachment proteins) (Clary et al., 1990; Waters et al., 1992; Whiteheart et al., 1993). Using NSF and a-SNAP, a complex of three proteins was isolated from brain extracts consisting of synaptobrevin 2 (vesicle associated membrane protein, VAMP2), syntaxin 1A and SNAP-25 (synaptosomal associated protein of $25 \mathrm{kDa}$ ). These proteins were called SNAP receptors (SNAREs) (Sollner et al., 1993). These SNAREs are specifically and individually cleaved by clostridial neurotoxins (tetanus and botulinum neurotoxins $A, B, C 1, D, E, F$ and $G$ ) (Jahn and Niemann, 1994; Montecucco and Schiavo, 1994). These neurotoxins impair neuronal exocytosis, demonstrating that each of the three SNAREs is essential for exocytosis. Synaptobrevin 2, syntaxin 1 and SNAP-25 serve as a paradigm for members of a SNARE complex because SNARE complexes that were found later have similar biochemical and biophysical features. 
Simultaneous studies using secretion mutants in yeast identified the proteins sec17p and sec18p (Novick et al., 1980) (Novick et al., 1981) as the factors responsible for membrane fusion. They turned out to be homologous to a-SNAP (Clary et al., 1990) and NSF (Wilson et al., 1989). In yeast exocytosis, proteins play a role that are very similar to the ones involved in neuronal exocytosis (Ferro-Novick and Jahn, 1994). Snc1p/2p (synaptobrevin 2 homologue) is localised on vesicles and Sso1p/2p (syntaxin1A homologue) and Sec9p/Spo20p (SNAP-25 homologue) resides on the plasma membrane.

The SNARE hypothesis postulates that intracellular fusion processes are mediated by specific combinations of SNARE proteins (Rothman and Warren, 1994). It was suggested that the transport vesicle contains the V-SNARE (vesicular SNARE), that the target membrane contains the t-SNARE (target SNARE) and that v- and t-SNAREs mediate membrane fusion by specifically interacting with each other. It was also postulated that NSF and a-SNAP dissociate the complex by ATP hydrolysis, thereby preparing the fusion step by structural rearrangement (Sollner et al., 1993). The SNARE hypothesis was later challenged by findings that showed that exchanging one or more SNAREs in complex formation is possible (Fasshauer et al., 1999).

The mechanism by which SNAREs mediate membrane fusion can be summarised as follows (Jahn et al., 2003). During membrane fusion SNARE proteins go through cycles of association and dissociation (Figure 1). In priming, SNARE complexes are disassembled by NSF and a-SNAP. The SNAREs are sorted to their appropriate membranes and are ready for subsequent rounds of fusion. Pre-complexes of Q-SNAREs assemble and cluster in lipid domains. In the tethering/docking step, which is mediated by proteins called tethering factors, the vesicle is positioned close to the target membrane. In the docking state, trans-SNARE complexes (SNAREs that reside in opposing membranes) are established. The formation of the SNARE complex is thought to proceed from the amino- to the carboxy-terminal end (zippering), a process which may provide the energy necessary to overcome the repulsion of the opposing membranes, thus resulting in membrane fusion. The SNAREs are now in a cis- 
complex (all SNAREs in one membrane) and need to be disassembled and recycled for a new round of fusion.

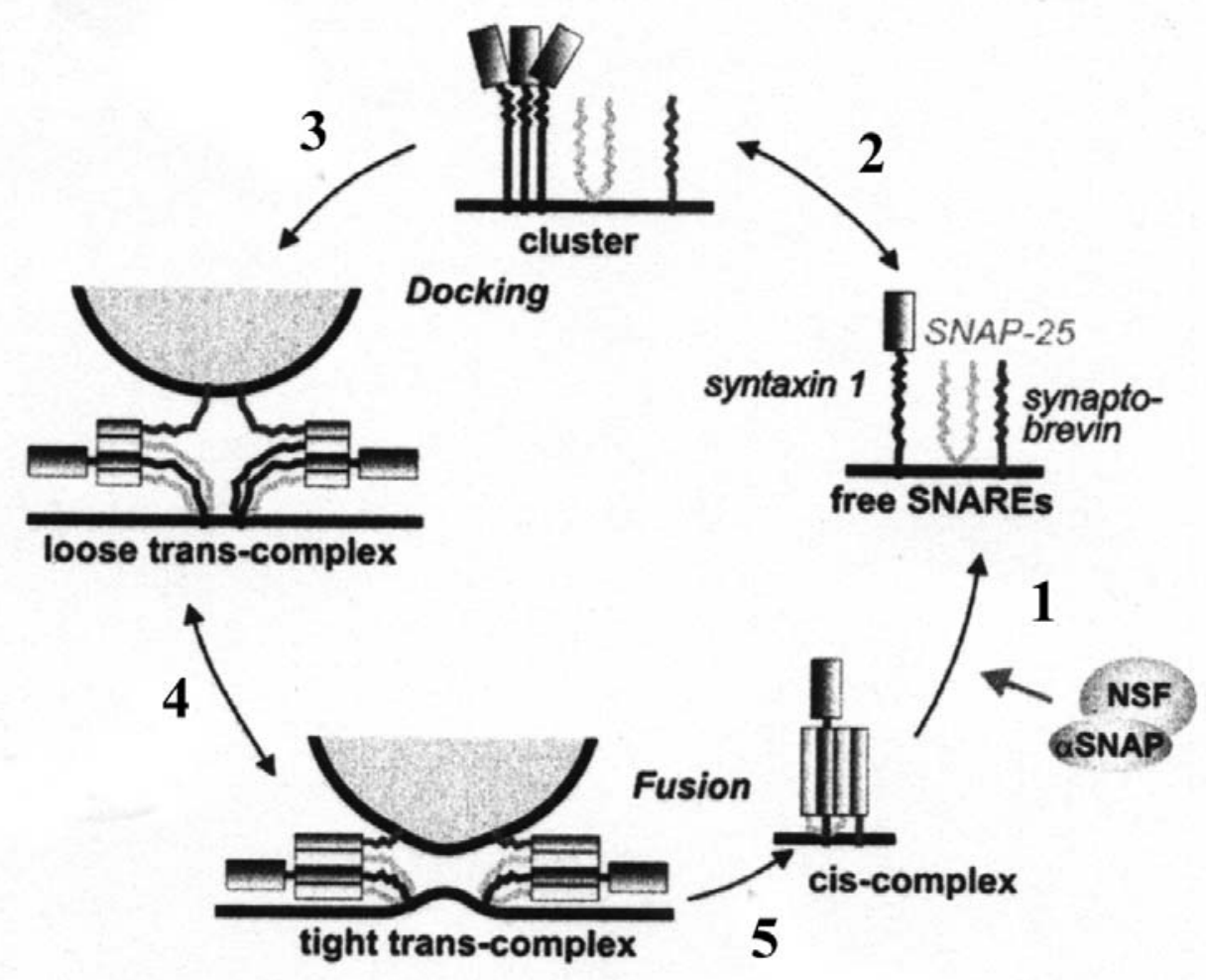

Figure 1 The SNARE Cycle. In the priming step the SNARE complex is disassembled by NSF and $\alpha$-SNAP and the SNAREs are present as monomers (1). In certain domains in the membrane clustering occurs (2). In the docking/tethering step the interaction of SNAREs is initiated by loose association of the amino-terminal domains in a trans-complex (3), in which the SNAREs are anchored in different membranes. Via zippering a tight transcomplex is formed (4). After fusion of the membranes the SNAREs are found in a cis-complex (5). The cycle begins again with disassembly of the complex (1). Figure modified from (Jahn et al., 2003)

Usually SNAREs consist of a SNARE motif of 60-70 amino acids length flanked by a variable amino-terminal domain and a carboxy-terminal transmembrane domain. However, there are some exceptions. Some SNAREs, like SNAP-25, SNAP-23 or ykt6p do not have a transmembrane domain but are bound to the membrane by palmitate or farnesyl anchors (Fukasawa et al., 2004; Hess et al., 1992; Vogel and Roche, 1999). Some SNAREs like the yeast vam7p or mammalian SNAP-29 are soluble and bind membranes via lipid binding 
domains such as the PX domain which binds to phosphoinositides (Boeddinghaus et al., 2002; Cheever et al., 2001).

SNAREs in solution are largely unstructured but upon complex formation a dramatic increase in ellipticity can be observed by CD spectroscopy, which indicates increased a-helix formation (Fasshauer et al., 1998a). Four SNARE motifs assemble into stable rod-like core complexes with a coiled-coil structure (Poirier et al., 1998). The SNARE motifs are composed of conserved heptad repeats that form 15 hydrophobic layers (layers denominated from -7 to +8 ). The residues in these layers are pointing to the inside of the complex perpendicular to the axis. They are the main points of interaction between the helices (Sutton et al., 1998). Only the unusual 0-layer contains ionic amino acids arginine or glutamine. Helix formation is thought to be the critical step leading to membrane fusion.

Two SNARE complexes have been described in detail biochemically and structurally: the neuronal and the late endosomal SNARE complex (Antonin et al., 2002b; Fasshauer et al., 1997). While the synaptic complex consists of three proteins (synaptobrevin 2, syntaxin 1 and SNAP-25) in a 1:1:1 ratio, with SNAP-25 contributing two helices, the late endosomal complex contains four proteins (endobrevin syntaxin 7 , syntaxin 8 and vti1b) in a 1:1:1:1 ratio. The proteins form a SDS resistant complex that migrates as one band in SDSPAGE (Antonin et al., 2000a; Hayashi et al., 1994). In addition, a SNARE complex including tomosyn (a regulatory protein with an R-SNARE motif) instead of synaptobrevin was characterised and crystallised (Hatsuzawa et al., 2003; Pobbati et al., 2004). This complex adapts the same overall structure than the other SNARE complexes. The core complex, the complex consisting of just the SNARE motifs, is thermo-stable and protease resistant. Crystal structure analysis of both complexes revealed a coiled-coil motif of four chains intertwined into a left-handed helix. Despite limited sequence homology the structures of the late endosomal complex and the neuronal complex are surprisingly similar and their biochemical and biophysical properties are comparable. The four helix bundle serves as a paradigm for SNARE complex 
structures since both complexes share it (Antonin et al., 2002b; Sutton et al., 1998).

As mentioned, SNAREs were originally classified as v- or t-SNAREs (Rothman, 1994). However, this classification is somewhat ambiguous because a t-SNARE may also be localised on a vesicle and vice versa, e.g. anterogradely transported ER-to-Golgi vesicles contain the (v-)SNARE Sec22p and the (t)SNARE Bos1p (Cao and Barlowe, 2000). In fact, sequence analysis revealed that most v-SNAREs are synaptobrevin homologues and contain an arginine in the centre of the SNARE motif, the 0-layer, and that most t-SNAREs contain a glutamine, leading to the reclassification of R- and Q-SNAREs after the one letter amino acid code. Further analysis revealed that the Q-SNAREs can be further subdivided into Qa-, Qb- and Qc-families (Bock et al., 2001; Fasshauer et al., 1998b). A SNARE complex usually consists of one R-SNARE and a $Q_{a} Q_{b} Q_{c}-S N A R E$ each (1R - 3Q rule). The positions of the $R$ - and $Q$ - residues can be swapped, with complete complex formation still occurring as long as the $1 \mathrm{R}-3 \mathrm{Q}$ ratio is maintained (Graf et al., 2005; Katz and Brennwald, 2000; Ossig et al., 2000).

\subsubsection{Endocytosis, Homotypic Fusion of Early Endosome and the Vacuole}

Higher eukaryotic cells have developed many different mechanisms by which fluids, particles, small molecules and macromolecules are taken up (Figure 2). This cargo is transported into the cell in vesicles surrounded by a single membrane. The general term for the mechanisms of this directed transport is endocytosis. It includes for example pinocytosis, phagocytosis, clathrindependent/receptor-mediated and clathrin-independent endocytosis. The best characterised mechanism is receptor mediated endocytosis: the first step of the process is the invagination of the plasma membrane, by the formation of a clathrin coated pit (Mukherjee et al., 1997). Clathrin coated vesicles (CCV) are pinched off and are transported to the cell interior. After shedding the clathrin coat the first compartment that they fuse with is the early endosome (Mayorga et al., 1988; Woodman and Warren, 1991). The early endosome (also called 
sorting endosome) is a sorting station from which there is direct transport back to the plasma membrane as for the transferrin receptor (Gruenberg and Howell, 1987). Other membrane proteins e.g. down regulated EGF-receptors, EGF or soluble proteins are transported to the late endosome and finally to the lysosome where they are degraded. Additionally, the early endosome exchanges proteins and membranes with the Golgi compartment. Early endosomes can fuse with other early endosomes (homotypic fusion) or with incoming endocytosed vesicles and the steady state equilibrium is maintained by continuous transport of vesicles to other organelles. Early endosomes send vesicles to the trans-Golgi-network (TGN) (Itin et al., 1997) and receive TGN derived vesicles (Cook et al., 2004). Two possibilities have been proposed for communication with late endosomes: early endosomes may be stable compartments from which vesicles travel to the late endosome (Aniento et al., 1993; Gruenberg et al., 1989); as an alternative, the maturation of early endosomes into multivesicular bodies or late endosomes was discussed (Dunn and Maxfield, 1992; Maxfield and McGraw, 2004). Furthermore, the late endosome has direct exchange of trafficking organelles with the TGN (Abazeed et al., 2005; Blanchette et al., 2004). Late endosomes, as well as lysosomes, can fuse homotypically (Luzio et al., 2000; Ward et al., 1997). 

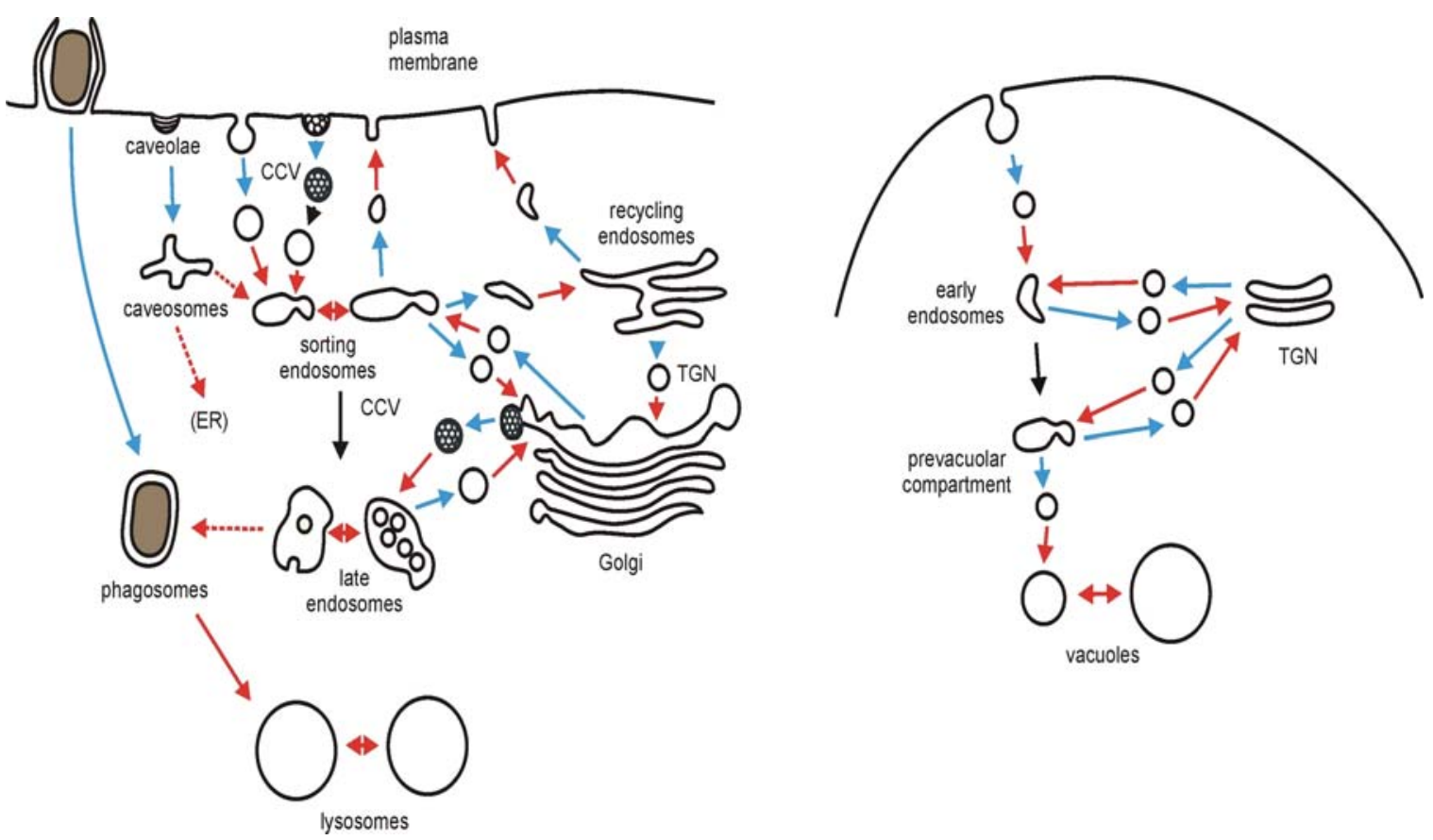

Figure 2 Intracellular Trafficking Routes. Depicted are the major mammalian (left) and yeast (right) endocytic pathways. Blue arrow, budding/fission followed by transport; red arrows, transport followed by fusion; black arrows, transport. CCV, clathrin-coated vesicle; TGN, trans-Golgi network. Figure from Brandhorst and Jahn (2005). 
An overview of the different SNAREs discussed in this study is given in Table1.

\begin{tabular}{|c|c|}
\hline Fusion Step & SNARE candidates \\
\hline \multicolumn{2}{|l|}{ mammals } \\
\hline EE-EE/CCV-EE & $\begin{array}{l}Q_{a} \text { syntaxin } 13, \text { syntaxin } 16 \\
Q_{b} \text { vti1a } \\
Q_{c} \text { syntaxin } 6 \\
R \quad \text { VAMP4 }\end{array}$ \\
\hline LE-LE & $\begin{array}{ll}Q_{a} & \text { syntaxin } 7 \\
Q_{b} & \text { vti1b } \\
Q_{c} & \text { syntaxin } 8 \\
R & \text { endobrevin }\end{array}$ \\
\hline Neuronal exocytosis & $\begin{array}{ll}Q_{a} & \text { syntaxin } 1 \\
Q_{b} & \text { SNAP-25 } \\
Q_{c} & \text { SNAP-25 } \\
R & \text { synaptobrevin }\end{array}$ \\
\hline \multicolumn{2}{|l|}{ yeast } \\
\hline ER-to-Golgi & $\begin{array}{ll}Q_{a} & \text { Sed5p } \\
Q_{b} & \text { Bos1p } \\
Q_{c} & \text { Bet1p } \\
R & \text { Sec22 } \\
\end{array}$ \\
\hline $\begin{array}{l}\text { Vac-Vac/ Prevacuole- } \\
\text { vacuole }\end{array}$ & $\begin{array}{l}Q_{a} \text { Vam3p } \\
Q_{b} \text { vti1p } \\
Q_{c} \text { Vam7p } \\
R \quad \text { Nyv1p, Ykt6p }\end{array}$ \\
\hline Intra Golgi & $\begin{array}{l}Q_{a} \text { Sed5p } \\
Q_{b} \text { Gos1p } \\
Q_{c} \text { Sftp } \\
R \text { Ykt6p }\end{array}$ \\
\hline Exocytotic & $\begin{array}{l}Q_{a} \text { Sso1/2p } \\
Q_{b} \text { Sec9p/Spo20p } \\
Q_{c} \text { Sec9p/Spo20p } \\
R \text { Snc } 1 / 2 p\end{array}$ \\
\hline
\end{tabular}

Table 1 SNAREs Involved in Different Fusion Steps in Mammals and Yeast. Listed are the SNARE candidates for certain fusion steps and their classification. EE, early endosome; LE, late endosome; $\mathrm{CCV}$, clathrin coated vesicle; ER, endoplasmic reticulum.

A special case of the endocytic pathway is the recycling of synaptic vesicles in neurons. Synaptic vesicles can go through hundreds of rounds of synaptic transmitter release (De Camilli and Takei, 1996). There are several models as to how this may happen (Sudhof, 2004). The models are not mutually exclusive and they may depend on the type of neuronal stimulation applied. The first model proposes the kiss and run mechanism. Synaptic vesicles fuse transiently 
with the membrane and the neurotransmitter is released through a fusion pore (Ceccarelli et al., 1973). The pore is then closed and the vesicle detaches from the plasma membrane to get recycled and refilled with neurotransmitter immediately. A second model proposes bulk endocytosis of membranes of which synaptic vesicles pinch off (Takei et al., 1996). Another scenario is the total collapse of the vesicle into the plasma membrane followed by recycling probably via clathrin mediated endocytosis. Clathrin coated vesicles were identified as intermediates of vesicle recycling using different methods (Heuser and Reese, 1973; Miller and Heuser, 1984; van der Bliek and Meyerowitz, 1991). After uncoating of the CCV the vesicle may go through endosomal sorting or it may get recycled directly. All of these models may involve clathrin dependent endocytosis except kiss and run.

Yeast cells have trafficking routes similar to mammalian cells (Figure 2). They generate endocytosed vesicles, early endosomal compartments, late endosomes/pre-vacuolar compartments, the TGN and the vacuole, (the equivalent to the mammalian lysosome) (Munn, 2000). As in the mammalian cell, endocytosed vesicles fuse with early endosomes, homotypic fusion of early endosomes is observed, early endosomes fuse with late endosomes, late endosomes fuse with the vacuole and homotypic vacuole fusion also takes place.

Early endosomal fusion in mammals and vacuole fusion in yeast are very well characterised fusion events. Short outlines of these processes are given in order to show parallels that exist in both.

Endosomal fusion is mediated and regulated by SNARE proteins and many other factors that play a role in tethering and recruiting the necessary elements. Fusion of endosomes is ATP and cytosol dependent (Braell, 1987; Diaz et al., 1988). Calcium is necessary for fusion and is released from the endosomal lumen (Holroyd et al., 1999; Mayorga et al., 1994). NSF and a-SNAP are enriched on endosomal membranes suggesting that these compartments are important as central sorting stations in membrane trafficking (Robinson et al., 1997). Rab5 is an essential factor for early endosomal fusion (Bucci et al., 1992; Gorvel et al., 1991). Rab5-GTP is hydrolysed continuously and therefore 
cannot bind stably to the membrane. Rab5 organises tethering by positive feedback loops. The first loop starts with the recruitment of Rab5 modifying proteins, so-called Rab5 effectors. The Rab-effectors Rabex- 5 and rabaptin- 5 activate Rab5 and stabilise it on the membrane by preventing GTP hydrolysis (Horiuchi et al., 1997; Stenmark et al., 1995). Active Rab5-GTP in turn recruits more Rababtin-5/Rabex-5 complex. In the second feedback loop Rab5-GTP interacts indirectly with the phosphatidyl-inositol-3-OH kinase p150/hVPS34 via Rabenosyn-5. This induces local production of phosphatidyl-inositol-3phosphate (PI(3)P) (Christoforidis et al., 1999b; Murray et al., 2002). PI(3)P binds proteins with FYVE finger domains (Gillooly et al., 2001; Stenmark et al., 2002). Rabenosyn- 5 is such a protein, and thus $\mathrm{PI}(3) \mathrm{P}$ production increases Rabenosyn- 5 concentration on the membrane. Another Rab5 effector with a FYVE finger domain is EEA1. EEA1 (early endosomal antigen 1) is a coiled-coil protein with two zinc-fingers and two Rab5 binding domains (Simonsen et al., 1998). EEA1 is required to be present on both donor and acceptor membrane for fusion to occur (Barbieri et al., 1998; Rubino et al., 2000). Thus EEA1 could be the tethering element in this fusion step. Rabenosyn- 5 was identified in a complex with the SM-protein vps45. The interaction of Rab5, vps45, PI(3)P, EEA1 may also be important for the recruitment of SNARE proteins for the final step of SNARE complex formation and fusion. A complex of EEA1, rabaptin-5, rabex-5, NSF and syntaxin 13 was postulated earlier (McBride et al., 1999). It was also shown that EEA1 binds syntaxin 13, and syntaxin 6 (Simonsen et al., 1999) and that vps45 binds syntaxin 16 (Dulubova et al., 2002). In the absence of vps45, syntaxin 16 is no longer able to bind to its SNARE partners (Bryant and James, 2001).

Vacuole fusion in yeast

Vacuole fusion can also be subdivided in the three steps of priming, docking and fusion. For priming, ATP, cytosol and salts are necessary (Conradt et al., 1994). Sec17p and sec18p (yeast homologues of $\alpha$-SNAP and NSF) disassemble the cis-SNARE complex consisting of vam3p (Qa), vam7p (Qc), vti1p $(\mathrm{Qb})$ and nyv1p $(\mathrm{R})$ or ykt6p $(\mathrm{R})$, thus providing the SNAREs for the fusion step (Mayer and Wickner, 1997; Mayer et al., 1996; Ungermann et al., 1998). In this priming step, the soluble SNARE vam7p is released from the complex into 
the cytosol. Docking includes ypt7-dependent tethering of the vacuoles and the formation of trans-SNARE complexes. Ypt7 (the Rab homologue) is activated through GDP-GTP exchange by the HOPS complex (homotypic vacuole fusion and protein sorting). The HOPS or VPS class $C$ complex consists of six subunits: vps11, $-16,-18,-33,-39,-41$. Sec17p and sec18p are able to dissociate this complex (Price et al., 2000; Sato et al., 2000). Vps11 and vps18 bind via a RING-Zinc-finger domain to vps 16 which binds the SM-protein vps33 (Rieder and Emr, 1997). Vps33 interacts with the $Q_{a}$-SNARE vam3p. Vps39 together with vps41 catalyse the nucleotide exchange of ypt7 (Wurmser et al., 2000).

Activated ypt7 is important for the following steps. Two Rho-GTPases, rho1p and cdc42p, are activated (Eitzen et al., 2001; Muller et al., 2001) and the SNARE proteins build the trans-complex. Vam7p is recruited to the membrane via ypt7 and 3-phosphoinositides (Boeddinghaus et al., 2002; Cheever et al., 2001; Ungermann et al., 2000) and fusion is mediated by the complete SNARE complex. The HOPS complex thus couples nucleotide exchange on ypt7 to effector recruitment and tethering, demonstrating similarities to the rabex5/rabaptin-5 complex that mediates Rab5 activation.

The tethering is still reversible before SNARE trans-complex formation, as shown by the inhibition of fusion by dilution. After trans-complex formation the vacuoles are irreversibly docked (Mayer and Wickner, 1997). The docking machinery as well as the SNAREs, the HOPS complex and ypt7 are now enriched at the contact points of the vacuoles in a ring called the vertex ring (Wang et al., 2003; Wang et al., 2002). This arrangement generates a disc-like membrane fragment that remains inside the vacuole after fusion. This mechanism is different from the formation of a fusion pore that dilates laterally as postulated for synaptic vesicle exocytosis. In the docking step calcium is released from the vacuole lumen and this enhances the binding of calmodulin to the membrane (Peters and Mayer, 1998; Ungermann et al., 1999). Calmodulin was found in a complex with protein phosphatase I. The protein phosphatase I was proposed to be essential in the final fusion step (Peters et al., 1999; Wickner, 2002) because phosphatase inhibitors also blocked fusion. Calmodulin was also shown to bind to the $V_{0}$ subunit of the vacuolar V-ATPase (Peters et 
al., 2001). It was proposed that trans-pairing of SNAREs enables the $V_{0}$ subunits to build $V_{0}$ trans-complexes themselves and that membrane fusion is mediated by this $\mathrm{V}_{0}-\mathrm{V}_{0}$ complex formation and not by the SNARE complex formation. This model suggests a role of SNAREs only for tethering (Mayer et al., 1996) because antibodies against SNAREs did not inhibit fusion after the docking state was established (Nichols et al., 1997). However, the fact that the vacuolar ATPase was not found at the vertex ring challenges the membrane fusion mediating role of $V_{0}$. The soluble SNARE vam7p is able to bypass the need for sec17, sec18 and ATP in vitro supporting the view that SNAREs are fusing the membranes (Thorngren et al., 2004). Adding recombinant vam7p rescued vacuolar fusion that was blocked by calcium chelators (Starai et al., 2005). This challenges the view that calmodulin acts downstream of the SNARE complex thus supporting the role of SNAREs as fusion mediators.

Studies on vacuole fusion in S. cerevisiae show similarities to early endosomal fusion in mammals. The mechanism by which Rab proteins orchestrate the recruitment of Rab effectors, SM proteins and SNAREs in the formation of the tethering machinery may be evolutionary conserved.

\subsection{Candidates for the Early Endosomal SNARE Complex}

Previously, syntaxin $13\left(Q_{a}\right)$ and syntaxin $6\left(Q_{c}\right)$ were found to be potential members of the SNARE complex involved in early endosomal fusion (Bock et al., 1997; Mills et al., 2001; Prekeris et al., 1998). Syntaxin 6 was found to colocalise with the Rab5 effector EEA1 on early endosomes (Simonsen et al., 1999). Other groups found an association of VAMP4 (R) with syntaxin 6 (Steegmaier et al., 1999) and vti1a-rp1 $\left(Q_{b}\right)$ with VAMP4, syntaxin 6, syntaxin 16 and syntaxin 5 (Xu et al., 1998) by coimmunoprecipitation. $F_{a b}$-fragments against vti1a were found to inhibit fusion of early endosomes (Antonin et al., 2000c). A SNARE complex containing a set of $Q_{a b c}$ and R-SNAREs, syntaxin 16 $\left(Q_{a}\right)$, vti1a $\left(Q_{b}\right)$, syntaxin $6\left(Q_{c}\right)$ and VAMP4 $(R)$ was identified by coimmunoprecipitation (Kreykenbohm et al., 2002) and it was suggested that this complex mediates early endosomal fusion. The involvement of endobrevin and vti1a was suggested by another study (Antonin et al., 2002a) using recombinant SNAREs as competitive inhibitors. Vti1 evolved in vertebrates as 
two isoforms vti1a and vti1b. Vti1b was identified as the $Q_{b}-S N A R E$ of the late endosomal SNARE complex. Vti1a was localised to synaptic vesicles and has a splice variant vti1a- $\beta$ (Antonin et al., 2000c). Another study suggested an involvement of the neuronal SNAREs SNAP-25 and synaptobrevin, which were found in a complex with syntaxin 13 (Sun et al., 2003). According to their intracellular localisation, potential R-SNAREs of the early endosomal complex are endobrevin (Antonin et al., 2000b), Ti-VAMP (also known as VAMP7) (Advani et al., 1999) and VAMP4 (Steegmaier et al., 1999) which are all found on early endosomes. While endobrevin is not present in brain, VAMP4 is ubiquitously expressed and it could therefore replace endobrevin as an RSNARE in the respective fusion reaction (Antonin et al., 2000a), while both RSNAREs could co-exist in parallel complexes in other tissues. The Q-SNAREs syntaxin 6 (Simonsen et al., 1999), syntaxin 7 (Prekeris et al., 1999), syntaxin 8 (Prekeris et al., 1999), syntaxin 10 (Tang et al., 1998a), syntaxin 11 (Valdez et al., 1999), syntaxin 13 (Prekeris et al., 1998; Tang et al., 1998b) and vti1a (Antonin et al., 2000c) were also found on endosomal compartments and are therefore potential members of the early endosomal complex. The conclusion that can be drawn from these conflicting data is that subcellular localisation of SNAREs is not enough to define their function. SNAREs might be members of more than one complex and functional data must be obtained in order to pinpoint the functional SNAREs that mediate a certain fusion step. An involvement of local regulatory factors that orchestrate SNARE activity in certain fusion steps cannot be excluded. Also, it appears that he most likely candidates according to in vivo and co-immunoprecipitation studies are syntaxin 13 or syntaxin $16\left(Q_{a}\right)$, vti1a $\left(Q_{b}\right)$, syntaxin $6\left(Q_{c}\right)$ and VAMP4 $(R)$.

\subsection{Liposomal Fusion, Compartmental Specificity and Topological Restriction}

An essential approach in investigating the role of SNAREs is the fusion of proteoliposomes. It has been shown by several groups that recombinant SNARE proteins can be reconstituted in liposomes and that SNAREs alone are sufficient to promote spontaneous fusion. SNARE-mediated fusion of liposomes was initially investigated using ER-to-Golgi yeast SNAREs (Weber et al., 1998). Similar experiments were also performed using the exocytotic neuronal 
SNAREs (Schuette et al., 2004), the mammalian late endosomal SNAREs, the yeast vacuolar and yeast exocytotic SNAREs (McNew et al., 2000; Parlati et al., 2000; Weber et al., 1998) all generating similar fusion efficiencies but different kinetics.

Four different methods can be used to investigate lipid bilayer fusion: (i) Counting of colocalised liposomes that are labelled with different dyes (bound to the lipids or to the internalised proteins) by a fluorescence microscopy approach. (ii) Lipid dequenching, in which the donor liposome contains two lipidattached dyes (NBD/Rhodamine) of which one quenches the other. Upon fusion with an (unlabelled) acceptor liposome, the dye concentration decreases, thus increasing the average distance between the dyes. This results in a signal increase of the previously quenched dye, (iii) Measurements of fluorescence resonance energy transfer (FRET) using two different dyes covalently attached to lipids. FRET occurs when the excitation and emission spectra of two dyes overlap. By exciting the donor dye, energy is transferred to the acceptor dye that emits light at its characteristic emission wavelength. This is only possible if the dyes are in close proximity. Initially, the dyes are separately inserted into two liposome populations; upon fusion, these lipids come in close proximity and FRET can be measured; (iv) Monitoring of FRET using carboxy-terminal labelled proteins that build FRET pairs when they assemble into the SNARE complex.

Each method has its advantages and disadvantages. The colocalisation assay depends on fluorescence microscopy with high resolution. The vesicles have to adhere quantitatively onto the cover slip. Also, it is difficult to distinguish docking from fusion purely on the basis of co-localisation. However, labelled proteins can be used to generate a FRET signal when they assemble into one complex. Fusion can be quantified and expressed as percent of colocalisation. Kinetic studies using this method are more difficult because for each time point a separate evaluation of colocalisation has to be prepared.

The dequenching assay relies on the spatial separation of the dye molecules by dilution into an acceptor membrane. Thus, the donor liposome needs to be used 
in lower amounts than the acceptor to provide acceptable signal to noise ratio. Multiple rounds of fusion may distort the result through the generation of higher dequenching signals. The quantification is difficult, because it relies on the dispersion of the liposomes by detergent resulting in a rather unreliable total dequenching signal. The total dequenching signal is not equal to the hypothetical total fusion event, as fusion of all liposomes with each other would result in a maximal signal that is lower than the signal created by detergent. However, the proteins do not need to be modified and the assay can be directly used to monitor fusion kinetics.

The 'lipid FRET assay' is convenient to use, and equal volumes of the liposome populations can be used. Reaction kinetics reach a plateau after a few rounds of fusion because the maximum FRET signal is then established. Therefore, evaluation of different reactions must be performed by comparing initial fusion rates. Finally, the carboxy-terminally labelled proteins can be used for colocalisation studies and also for the recording of fusion kinetics. FRET signal is generated when the proteins are assembled into one complex. The drawback of this method is the time consuming generation of cysteine mutants and their labelling.

Using liposomal fusion assays, several studies have suggested the compartmental specificity of SNARE proteins i.e. it was claimed that SNAREs have the intrinsic property of only interacting specifically with the appropriate (cognate) partner SNAREs from certain cellular compartments (McNew et al., 2000; Paumet et al., 2001; Paumet et al., 2004). The combination Sed5-Bos1Sec22 for example only fuses with Bet1p liposomes (Parlati et al., 2000) but not with Sftp or Tlg1p liposomes. However, Sftp and Tlg1p can fuse with Sed5p if this molecule is combined with different SNAREs. Judging from these experiments it seems that there is no promiscuity but more of a fine-tuning of interaction of SNAREs in different combinations (Pelham, 2001). In a different case, however, compartmental specificity was lacking: The yeast plasma membrane R-SNAREs Snc1p and Snc2p can be replaced by the ER-to-Golgi and vacuolar R-SNAREs sec22 $p$ and nyv $1 p$ when fusing with liposomes 
containing the plasma membrane SNAREs $\operatorname{Sso1p}\left(Q_{a}\right)$ and $\operatorname{Sec} 9 p\left(Q_{b c}\right)$ (McNew et al., 2000).

Several other experiments suggest that promiscuous behaviour is possible in vivo: endocytic vesicles in yeast containing Snc1 usually fuse with early endosomal organelles positive for Tlg2p (Gurunathan et al., 2000; Lewis et al., 2000; Seron et al., 1998). However, if Tlg2p is missing they fuse with a late endosomal compartment containing Pep12p instead. If both Tlg2p and Pep12p (and vam $3 p$ ) are missing the vesicle can also fuse with Golgi membranes containing Sed5p (Holthuis et al., 1998). Other studies showed that Pep12p can be replaced by vam3p in yeast (Darsow et al., 1997; Gotte and Gallwitz, 1997) and if Sec22p is missing ykt6p is up-regulated and replaces sec22p in the ERto-Golgi transport (Liu and Barlowe, 2002). In homotypic vacuole fusion nyv1 can be replaced by ykt6p, and SNAP-23 can replace SNAP-25 in the exocytosis of secretory granules in chromaffin cells (Sorensen et al., 2003). In Drosophila the two isoforms of synaptobrevin syb and n-syb are be able to functionally replace each other in the compound eye. In the same experiment rat VAMP2 and cellubrevin were able to substitute for the endogenous synaptobrevins when over-expressed (Bhattacharya et al., 2002). In chromaffin cells synaptobrevin 2 and cellubrevin are able to functionally replace each other (Borisovska et al., 2005).

The topological restriction of SNARE mediated fusion was suggested (Parlati et al., 2000) using ER-to-Golgi SNAREs in the liposomal dequenching assay. The topology would ensure that fusion only occurs if the SNAREs are distributed in correct combinations over the two opposing membranes. It was suggested that fusion only occurs if the v-SNARE Bet1p is present in one membrane and the three t-SNAREs Sed5-Bos1-Sec22 are reconstituted in the other membrane. No other fusogenic combination with these proteins was observed (Parlati et al., 2000).

\subsection{Aims of this Study}

The goal of this study was to identify the SNARE proteins mediating homotypic early endosomal fusion. This work was supported by D. Brandhorst who 
worked in parallel on the development of a new in vitro assay for early endosome fusion (Brandhorst PhD thesis, 2004). The work was based on the results from both our group and the work of others who identified potential members of this complex. The goal of the project was to generate the appropriate tools i.e. recombinant proteins and antibodies to allow the identification of the SNAREs involved in early endosomal fusion. The SNARE complex was then to be characterised biochemically and structurally, with aspects of compartmental specificity and topological restriction also being investigated. 


\section{Materials and Methods}

\subsection{Chemicals}

Acrylamide/Bisacrylamide solution, Roth (Karlsruhe, Germany)

Ammoniumpersulfate (APS), Sigma (Deisenhofen, Germany)

Bradford-Reagent, Biorad (Richmond, USA)

Coomassie Brilliant Blue R-250, Serva (Heidelberg, Germany)

Cholat $3 \alpha 7 \alpha 12 \alpha$ Trihydroxy $5 \beta$ cholan 24oic acid sodium salt, Sigma

(Deisenhofen, Germany)

Dithiothreitol (DTT), Roth (Karlsruhe, Germany)

EDTA Titriplex III, Merck (Darmstadt, Germany)

Glycine, Sigma (Deisenhofen, Germany)

Imidazole, Sigma (Deisenhofen, Germany)

Isopropyl- $\beta$-D-thiogalactoside (IPTG), Roth (Karlsruhe, Germany)

N,N,N',N'- Tetramethylethylendiamine (TEMED), Biorad (Richmond, USA)

Protease K, Boehringer (Mannheim, Germany)

Sodiumdodecylsulfate (SDS), Biorad (Richmond, USA)

Tris (hydroxymethyl)-aminomethane (Tris), Merck (Darmstadt, Germany)

Triton X-100, Merck (Darmstadt, Germany)

Trypsin, Sigma (Deisenhofen, Germany)

Urea, Merck (Darmstadt, Germany)

Lipids, Avanti Polar lipids (Alabaster, Al, USA)

Cholesterol (brain)

L- $\alpha$-Phosphatidylcholine (brain)

Phosphatidylethanolamine (brain)

Phosphatidylserine sodium salt (brain)

Phosphatidylinositol sodium salt (bovine liver)

Oregon Green® 488 DHPE $\quad$ Oregon Green $₫ 488$ 1,2-dihexadecanoyl-sn glycero-3-phosphoethanolamine

Texas Red® DHPE Texas Red® 1,2-dihexadecanoyl-sn-glycero-3phosphoethanolamine, triethylammonium salt 
Rhodamine DHPE

NBD-PE
Lissamine ${ }^{\mathrm{TM}}$ rhodamine B 1,2-dihexadecanoylsn-glycero-3-phosphoethanolamine, triethylammonium salt $\mathrm{N}$-(7-nitrobenz-2-oxa-1,3-diazol-4-yl)-1,2dihexadecanoyl-sn-glycero-3phosphoethanolamine, triethylammonium salt

Lipid Mix

PC, PE, PS, PI, Cholesterol (Phosphatidylcholine: Phosphatidylethanolamine:

Phosphatidylserine: Phosphatidylinositol: Cholesterol) in a molar ratio of PC:PE:PS:PI:Chol, 5:2:1:1:1 with a final concentration of $27 \mu \mathrm{mol}$ total lipid $/ \mathrm{ml}$.

Bacteria stains (Stratagene, La Jolla, CA, USA)

Escherichia coli BL21 (DE3) B F $\mathrm{F}^{-}$dcm ompt hsdS $\left(\mathrm{rB}^{-} \mathrm{mB}^{-}\right)$gal $\lambda(\mathrm{DE} 3)$

Escherichia coli XL-1-Blue recA1, endA1, gyrA96, thi', hsdR17, (rk', mk+),

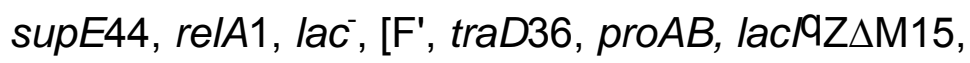
$\left.\operatorname{Tn} 10\left(\operatorname{tet}^{\mathrm{r}}\right)\right]$

Escherichia coli JM109 e14-(McrA-) recA1 endA1 gyrA96 thi-1 hsdR17 ( $r \mathrm{~K}^{-}$ $m \mathrm{~K}+$ ) supE44 relA1 $\triangle\left(\right.$ lac-proAB) $\left[\mathrm{F}^{\prime}\right.$ traD36 proAB laclqZ $\Delta M 15]$.

Other

ÄKTA - system, Amersham Pharmacia Biotech (Uppsala, Sweden)

FluoroMax-2, Jobin Yvon-Spex (Edison, NJ, USA)

Dawn DSP and Optilab DSP, Wyatt Technology Corporation (MALLS)

Spectroplarimeter J-720, Jasco (CD-spectroscopy)

Software

Sigma Plot 2001, for Windows Version 7.101

ASTRA software version 4.7

Jasco, Spectra manager for Windows 95/NT version 1.53.00, CD spectroscopy 


\subsection{Standard Protocols}

\section{Determination of Protein Concentration}

Protein concentration was determined according to Bradford (Bradford, 1976). A set of standards containing $1,2,3,4,5 \mu \mathrm{g}$ bovine serum albumin and the proteins were diluted in $200 \mu \mathrm{l} \mathrm{dH}_{2} \mathrm{O}$ and then mixed with $800 \mu \mathrm{l}$ Bradford solution. After incubation for $5 \mathrm{~min}$ at RT the absorbance at $595 \mathrm{~nm}$ wavelength was measured using a photometer. The protein concentrations of interest were obtained from interpolation onto the linear trace obtained from the standards.

\section{Preparing and Running the SDS-PAGE Gels}

The SDS-PAGE was performed as described by Schaegger and von Jagow (Schagger and von Jagow, 1987). The loading buffer contained 50mM Tris/ $\mathrm{HCl}$, $4 \%(w / v)$ SDS, $0.01 \%$ Serva Blue G, $12 \%(w / v)$ Glycerol, $2 \%(v / v) \beta$ mercaptoethanol, pH 6.8 .

\section{Coomassie-blue-staining}

After electrophoresis the stacking gel (Schagger and von Jagow, 1987) was discarded and the separation gel was stained for $15-30 \mathrm{~min}$ in $50 \%(\mathrm{v} / \mathrm{v})$ methanol, 10\% (v/v) acetic acid and 0.2\% (w/v) Coomassie Brilliant Blue R-250 under agitation. The gel was destained in $50 \%(\mathrm{v} / \mathrm{v})$ ethanol and $10 \%(\mathrm{v} / \mathrm{v})$ acetic acid for $15 \mathrm{~min}$ and then in 10\% (v/v) ethanol and 5\% (v/v) acetic acid until no background staining was visible. After scanning, the gel was dried in a gel dryer, wrapped in cellophane foil, and preserved for further analysis.

\subsection{Molecular Cloning}

Cloning was performed according to standard protocols (Sambrook, 2001). Enzymes for DNA manipulations were obtained from New England Biolabs (Beverly, MA), and Ni-NTA agarose from Qiagen (Hilden, Germany), Oligonucleotides were obtained from MWG Biotech AG (Ebersberg, Germany). Unless indicated otherwise, all other reagents were purchased from Sigma (Deisenhofen, Germany). Plasmid manipulations were performed using the 
Escherichia coli strain XL1-Blue and protein expression in strain BL21DE3 and JM109.

Syntaxin 6 (1-232) cDNA in pGEX vector was kindly provided by R.H. Scheller (San Francisco) (Bock et al., 1996). The transmembrane region was attached by PCR using the oligonucleotide sx6TMDext 5'ccagtgatcggcgccaatggtgtgccatagccatcctctttgcagtcctgttggttgtgctcatcctcttcttagtg ctgtga3', and as flanking primers the oligonucleotides sx6_for_Nhe 5'aaagctagcatgtccatggaggaccccttc3' (forward) and sx6_rev_Xho 5'aaactcgagtcacagcactaagaagaggatg3' (reverse). The core fragment was amplified from the original clone using the oligonucleotide sx6core_169234_BamHI_for 5'aaaggatccatgcaggatgagcagttgg3' (forward) and sx6core_169234_HindIII_rev 5'tattttaagcttttattggcgccgatcactgg3' (reverse).

Syntaxin 13 pGEX 1-232 (Antonin et al., 2000) was subcloned in pET28a using sx13_for_EcoRI 5'gggaattccatatgtcctacggtcccttagac3' (forward) and sx13_rev_Xho 5'atactcgagcacatcttcttgcgag3' (reverse). The oligonucleotide sx13TMDext

5'cgcaagaagatgtgtatcctcgtgcttgtcctctcagtgattgttacagtcttggtagttgttatctgggttgcttcta agtga3' was used to amplify the transmembrane region as described above with the flanking primers sx13_1-274_for_Nde 5'gggaattccatatgtcctacggtcccttagac3' (forward) and sx13_1-274_rev_Xho 5'aaactcgagtcacttagaagcaaccc3' (reverse). The core fragment 182-250 was amplified using the primers sx13_core_for_Nde1 5'aacttattcatatgagggaaacg3' (forward) and sx13_rev_Xho 5'atactcgagcacatcttcttgcgag3' (reverse).

The syntaxin 16 clone was provided by G. Fischer von Mollard (University, Göttingen) (Kreykenbohm et al., 2002). DNA encoding syntaxin 16 core fragment (residues 237-302) was generated by PCR using the primers sx16_237-302_for_BamHI 5'aaaggatccatgagggagcgagagatcc3' (forward) and sx16_237-302_rev_HindIII 5'tttcaagctttcacttccgattcttcttctggtgc3' (reverse).

Vti1a 1-217 was provided by G. Fischer von Mollard in pGEM-Teasy (cloning vector, Promega). After amplification of the coding region using the primers vti1a1-217_for_Nde 5'ggaattccatatgtcagccgacttcgaaggg3' (forward) and Vti1a1-217_rev_Xho 5'tccgctcgagtcagtgtcctctgacaaaaaaag3' (reverse), the DNA was subcloned into pET28a. This clone was used as a template to amplify a Vti1a 1-192 clone with the primers vti1a_1-192_for_EcoRI 
5'gggaattccatatgtcagccgacttcgaaggg3' (forward) and vti1a_1-192_rev_HindIII 5'aaagaagctttcagcggttttggatgattcttcg3' (reverse).

Rat VAMP4 (coding for residues 1-117 and 47-117) in pGEX-KG was provided by W. Antonin (MPI-bpc, Göttingen). cDNA encoding full-length VAMP4 (residues 1-141) was amplified via RT-PCR using AccessQuick RT-PCR System kit from Promega from a total RNA rat brain library (kindly provided by S. Takamori, MPI-bpc, Göttingen) using the following oligonucleotides: VAMP4_1-141_for_Ndel 5'aaacatatgcctcccaagttcaagcgc3' (forward) and VAMP4_1-141_rev_Xhol 5'aaactcgagtcaagtacggaatttcacaac3' (reverse). All constructs were subcloned into the pET28a vector. Each clone was verified by DNA sequencing.

\subsection{Expression and Purification of Recombinant Proteins}

All recombinant proteins were expressed in shaking cultures of $E$. coli strains BL21DE3 and JM109 as His $_{6}$-tagged or GST-tagged fusion proteins and purified by $\mathrm{Ni}^{2+}$-agarose or $\mathrm{GSH}-$ sepharose, respectively. The bacteria were harvested by centrifugation and the bacterial pellets containing a $\mathrm{Ni}^{2+}$ tagged protein (of $6 \times 700 \mathrm{ml}$ TB (for His 6 -tagged proteins) or LB medium (for GSTtagged proteins)) were resuspended in $\mathrm{Ni}^{2+}$-wash buffer $(20 \mathrm{mM} \mathrm{Tris} / \mathrm{HCl}, \mathrm{pH}$ $7.4,500 \mathrm{mM} \mathrm{NaCl}, 8 \mathrm{mM}$ Imidazole) (for transmembrane proteins cholate was added to a final concentration of $5 \% \mathrm{w} / \mathrm{w}$ ). Bacteria expressing GST fusion proteins were resuspended in GST wash buffer $(20 \mathrm{mM}$ Tris/ $\mathrm{HCl}, \mathrm{pH} 7.4$, $500 \mathrm{mM} \mathrm{NaCl}, 1 \mathrm{mM}$ DTT, $1 \mathrm{mM}$ EDTA). To break open the cells $1 \mathrm{mg} / \mathrm{ml}$ lysozyme, $1 \%$ Triton X-100 (v/v), $1 \mathrm{mM} \mathrm{PMSF,} 1 \mathrm{mM} \mathrm{MgCl} 2$ and a spatula tip DNase I were added and the extract was incubated for $10 \mathrm{~min}$ at RT. All following steps were performed at $4^{\circ} \mathrm{C}$ or on ice. Additional ultrasound pulses of $3 \times 40$ s were performed. If the protein precipitated urea to a final concentration of $4 \mathrm{M}$ was added. The suspension was mixed with a blender for several minutes. This mix was then centrifuged for $10 \mathrm{~min}$ at $12,000 \mathrm{~g}$ (Beckman SS-34 rotor). The supernatant was transferred to fresh tubes and centrifuged again under the same conditions to ensure that all cell debris were removed. The pellet was discarded and the supernatant incubated with Ni-NTA beads

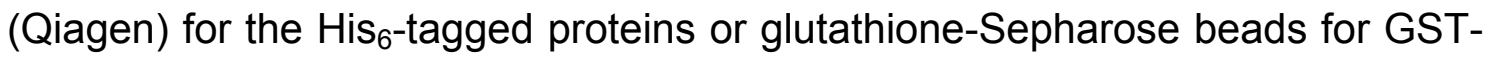
tagged proteins (following manufacturer's instructions) rotating, for 1-2 $\mathrm{h}$. This 
mix was then poured into a column ( $15 \mathrm{~cm}$ length, $3 \mathrm{~cm}$ width) and the beads were allowed to settle. The beads were then washed three times with $100 \mathrm{ml}$ washing buffer. The protein was then eluted with 3 bed volumes of elution buffer (20mM Tris/HCl, pH 7.4, 500mM NaCl, $400 \mathrm{mM}$ imidazole for His ${ }_{6}$-tagged proteins; $20 \mathrm{mM}$ Tris $/ \mathrm{HCl}, 500 \mathrm{mM} \mathrm{NaCl}, 10 \mathrm{mM}$ glutathione for GST fusion proteins). The proteins were dialyzed for a total of $16 \mathrm{~h}$ at $4^{\circ} \mathrm{C}$ against dialysis buffer $(20 \mathrm{mM}$ Tris/ $/ \mathrm{HCl}, 50 \mathrm{mM} \mathrm{NaCl}, 1 \mathrm{mM}$ DTT, $1 \mathrm{mM}$ EDTA) (containing $1.5 \%$ cholate $(\mathrm{w} / \mathrm{v})$ for transmembrane proteins) using Spectra Por molecularporous membranes (Spectrum) with appropriate molecular weight cut off. The tags were removed by digestion with thrombin $(20 \mu \mathrm{l}$ of a $4 \mathrm{U} / \mu \mathrm{l}$ stock on $30 \mathrm{ml}$ eluate) in the dialysis tube. An amount of $5 \mu \mathrm{g}$ total protein was analyzed on a SDSPAGE to check purity. All proteins were further purified using Mono-Q or Mono$S$ columns on a FPLC system (Amersham Pharmacia Biotech, Uppsala, Sweden). After dialysis the protein was loaded on an ion-exchange column, washed with several column volumes and then eluted with a linear gradient increasing salt concentration from $0 \mathrm{mM}$ to $1000 \mathrm{mM} \mathrm{NaCl}$ in $20 \mathrm{mM}$ Tris $/ \mathrm{HCl}$ buffer, pH 7.4 with $1 \mathrm{mM}$ EDTA and $1 \mathrm{mM}$ DTT. The protein was collected in fractions and each peak fraction was analysed via SDS-PAGE. The fractions were pooled according to their purity and then shock frozen in liquid nitrogen and preserved at $-80^{\circ} \mathrm{C}$.

\subsection{Polyclonal Antibodies}

Rabbit antisera were generated using purified cytosolic fragments (SNARE protein lacking the transmembrane region) of VAMP4, syntaxin 6 , syntaxin 13, and vti1a as antigens. Rabbits were immunised by injecting an initial amount of $300 \mu \mathrm{g}$ and then $150 \mu \mathrm{g}$ of protein every four weeks several times. All antisera recognised single bands of the expected molecular mass in immunoblots of PC12 or NRK cell homogenates. All antibodies were affinity-purified using the respective purified proteins that were covalently bound to CNBr-Sepharose (Amersham-Pharmacia Biotech, Uppsala, Sweden), following the manufacturer's instructions. 


\subsection{Assembly of the Early Endosomal SNARE Complex}

Assembly was performed according to Antonin (Antonin et al., 2000a). The core fragments of VAMP4, syntaxin 6 , syntaxin 13 and vti1a were mixed in a 1:1:1:1 molar ratio with a total amount of approximately $20 \mathrm{mg}$ protein. The mixture was then dialysed overnight in $20 \mathrm{mM}$ Tris/ $\mathrm{HCl}$ buffer, $\mathrm{pH} 7.4$ with $1 \mathrm{mM}$ EDTA, 1mM DTT and $1 \mathrm{M} \mathrm{NaCl}$. The buffer was then changed to $20 \mathrm{mM}$ Tris/HCl buffer, $\mathrm{pH}$ 7.4 with $100 \mathrm{mM} \mathrm{NaCl}$ and $2 \mathrm{M}$ urea. After dialysis the complex was purified using ion-exchange chromatography (MonoQ) and the peak fractions were analysed by SDS-PAGE.

\subsection{Dissociation of the SNARE Complex by NSF}

Complex disassembly was performed according to Hayashi (Hayashi et al., 1995). The SNARE complex $(2.5 \mu \mathrm{M}$ final concentration) was incubated with NSF $(2 \mu \mathrm{M}), \alpha-S N A P(35 \mu \mathrm{M}), \operatorname{ATP}(2.5 \mathrm{mM})$ and $\mathrm{MgCl}_{2}(2 \mathrm{mM})$ or EDTA $(1 \mathrm{mM})$ and the mix was adjusted to $500 \mathrm{mM}$ or $1000 \mathrm{mM} \mathrm{NaCl}$ in $20 \mathrm{mM}$ Tris/HCl buffer. The reaction was incubated for $1 \mathrm{~h}$ at $37^{\circ} \mathrm{C}$. NSF and $\alpha$-SNAP were kindly provided by Ulrike Winter (MPI-biophysical chemistry, Göttingen).

\subsection{Circular Dichroism Spectroscopy}

CD spectroscopy was performed according to Fasshauer (Fasshauer et al., 1999) using a Jasco model J-720 photometer (Jasco Corporation, Tokyo, Japan) upgraded to a J-715U and equipped with a 6-Position Peltier Effect Cell Changer. Far UV CD spectra were obtained by averaging 5 scans using steps of $0.2 \mathrm{~nm}$ with a scan rate of $50 \mathrm{~nm} / \mathrm{min}$. A buffer containing $20 \mathrm{mM}$ Tris/ $\mathrm{HCl}, \mathrm{pH}$ $7.4,500,750$ or $1000 \mathrm{mM} \mathrm{NaCl}$ or 2,4 or $6 \mathrm{M}$ urea were used. The measurements were performed in Hellma quartz cuvettes with path lengths of $1 \mathrm{~mm}$. Spectra were taken in the range from 190 to $250 \mathrm{~nm}$. For the thermal melt experiment the wavelength was set to $222 \mathrm{~nm}$, the temperature to $4^{\circ} \mathrm{C}$. Then the measurement was started by gradually increasing the temperature to $98^{\circ} \mathrm{C}$ at a rate of $60^{\circ} \mathrm{C} / \mathrm{h}$. Subsequently the temperature was again to $4^{\circ} \mathrm{C}$ at a rate of $60^{\circ} \mathrm{C} / \mathrm{h}$. 


\subsection{Multi Angle Laser Light Scattering (MALLS)}

Multi angle laser light scattering (MALLS) was performed to determine the molecular size of the SNARE complex. It was performed according to Antonin (Antonin et al., 2000a). A gel filtration column (Superdex 200 HR-10/30) (Amersham Pharmacia) was equilibrated to $500 \mathrm{mM}$ or $1000 \mathrm{mM} \mathrm{NaCl}$ in $20 \mathrm{mM}$ Tris/HCl buffer, pH 7.4 with 1mM DTT, 1mM EDTA, 2M urea and the complex was dialysed against the same buffer. $500 \mu \mathrm{l}$ of the complex $(6 \mathrm{mg} / \mathrm{ml})$ were injected into the column and the MALLS measurement was started at a flow rate of $0.5 \mathrm{ml} / \mathrm{min}$. The elution was monitored by UV-absorption at $280 \mathrm{~nm}$, light scattering at 632.8nm (Dawn Instrument, Wyatt Technology) and differential refractometry (Optilab Instrument, Wyatt Technology). The data were evaluated using the ASTRA software (Wyatt Technology). The change of the refraction index as a function of the concentration $(\mathrm{dn} / \mathrm{dc})$ is nearly constant for proteins and a value of 0.189 was used for data analysis (Wen et al., 1996).

\subsection{Crystallisation, Diffraction Data Collection, Structure Solution and Refinement}

Crystallographic work was conducted in collaboration with Markus Wahl, MPIbpc, Göttingen.

To this end the early endosomal SNARE complex was prepared in $20 \mathrm{mM}$ Tris/HCl, pH 7.4, 500 mM NaCl, 1 mM EDTA, 1 mM DTT at a concentration of 6 $\mathrm{mg} / \mathrm{ml}$. Crystallisation trials were conducted at RT and $4^{\circ} \mathrm{C}$ in sitting drop vapour diffusion format. Initial screens made use of commercial reservoir collections (Crystal Screen 1 and 2, Crystal Screen Cryo, PEG/lon Screen and Grid Screens from Hampton Research) and automated dispensing of 200nl drops (100nl protein plus 100nl reservoir) in 96-well trays. Initial conditions producing micro crystals were subsequently optimised manually $(2 \mu$ protein plus $2 \mu l$ reservoir drops) by systematic variation of chemical components in 24-well trays. Thin crystal plates were ultimately obtained with a reservoir solution of 0.1 $\mathrm{M}$ tri sodium citrate dihydrate, $\mathrm{pH}$ 5.6, 36\% (v/v) 2-methyl-2,4-pentandiol, 0.2M $\mathrm{Li}_{2} \mathrm{SO}_{4}$ at $\mathrm{RT}$. 
Crystals could be flash frozen in liquid nitrogen without additional cryoprotection. Diffraction data were collected at $100 \mathrm{~K}$ at the PXII beamline of the Swiss Light Source (Villigen, Switzerland) on a MarResearch CCD detector. Data from five crystals had to be combined to arrive at a complete data set (Table 4). Diffraction data were processed with the HKL package.

The structure of the complex was solved by molecular replacement (Molrep; (1994)) using the late endosomal SNARE complex (PDB entry 1GL2) (Antonin et al., 2002b) as the search model. After initial rigid body and positional refinement with CNS (Brunger et al., 1998), the protein sequences were adjusted by manual model building with MAIN (www-bmb.ijs.si/doc/index.html). Refinement was completed by alternating between refinement cycles (simulated annealing, positional and B-factor refinement) and manual inspection. In the final stages of refinement, water oxygens were manually positioned into vacant spherical peaks of the $F_{0}-F_{c}$ difference electron density map. During all refinement steps, $5 \%$ of the reflections were set aside for monitoring of the $R_{\text {free }}$ factor. Refinement converged at a $R_{\text {work }}$ of $25.2 \%$ and a $R_{\text {free }}$ of $29.6 \%$ maintaining good stereochemistry (Table 5).

\subsection{Preparation of Lipid Micelles}

Liposomes were prepared according to Schuette (Schuette et al., 2004). The lipids were first mixed in chloroform/methanol, 2:1 in the following molar ratio: PC:PE:PS:PI:cholesterol 5:2:1:1:1. For the NBD/Rhodamine liposomes 1.5\% $(\mathrm{v} / \mathrm{v})$ of NBD and Rhodamine were added to the lipid mix. The phosphatidylinositol (PE)-Texas red or PE-Oregon green liposomes contained $3 \%(\mathrm{v} / \mathrm{v})$ of the respective dye. After removal of the solvent the lipids were solubilised in a final concentration of $13.5 \mathrm{mM}$ in the micellar buffer HB100 (20mM Hepes/KOH, pH 7.4, $100 \mathrm{mM} \mathrm{KCl}, 1 \mathrm{mM} \mathrm{DTT})$ with $5 \%$ cholate (w/v). The micelles were aliquoted into Eppendorf cups under Argon and stored at $-80^{\circ} \mathrm{C}$.

For fusion assays, a lipid/protein ratio of 100:1 in the liposomes for all integrated proteins was used. $100 \mu \mathrm{l}$ or $50 \mu \mathrm{l}$ of lipid micelles $(1.35 \mu \mathrm{mol}$ or $0.675 \mu \mathrm{mol}$ of lipid) were mixed with $15 \mathrm{nmol}$ or $7.5 \mathrm{nmol}$ of each SNARE protein in a total volume of maximally $200 \mu \mathrm{l}$ or $100 \mu \mathrm{l}$, respectively. The early endosomal SNAREs were mixed in a 1:1:1:1 molar ratio, combined with the lipids and 
incubated either $1 \mathrm{~h}$ at $\mathrm{RT}$ or over night at $4^{\circ} \mathrm{C}$ before generating the liposomes. Liposomes were formed by detergent removal by applying the protein-lipid mix to a Biorad EconoColumn of $0.7 \mathrm{~cm}$ diameter and $15 \mathrm{~cm}$ length packed with Sephadex G50 superfine which was equilibrated with HB1000 without detergent. After about $1.2 \mathrm{ml}$ of flow through, the protein-containing liposomes eluted in about $500 \mu l$. Aggregation was checked by centrifugation in a table-top centrifuge for $10 \mathrm{~min}$ at $13,000 \mathrm{rpm}$. In case of aggregation a pellet was visible. The supernatant was transferred to a fresh tube and protein concentration was determined according to Bradford (Bradford, 1976).

\subsection{Determination of Orientation of SNAREs in Liposomes}

The correct orientation of SNARE was verified by proteolytic digest followed by SDS PAGE analysis. The liposomes were digested with trypsin in the presence or absence of Triton X-100. Non-degraded protein was expected to be visible in the case of digest without Triton. The proteins would stay intact if the proteins were oriented to the lumen of the liposome because Triton does not cross the membrane. The Q-SNAREs and VAMP4 were digested almost completely in the absence of detergent indicating complete outside-out orientation (Figure 3). VAMP4 exhibits a dimer. Endobrevin remains partially undigested without Triton indicating partial outside-in orientation. However, endobrevin was fully degraded after solubilisation of the liposomes. 


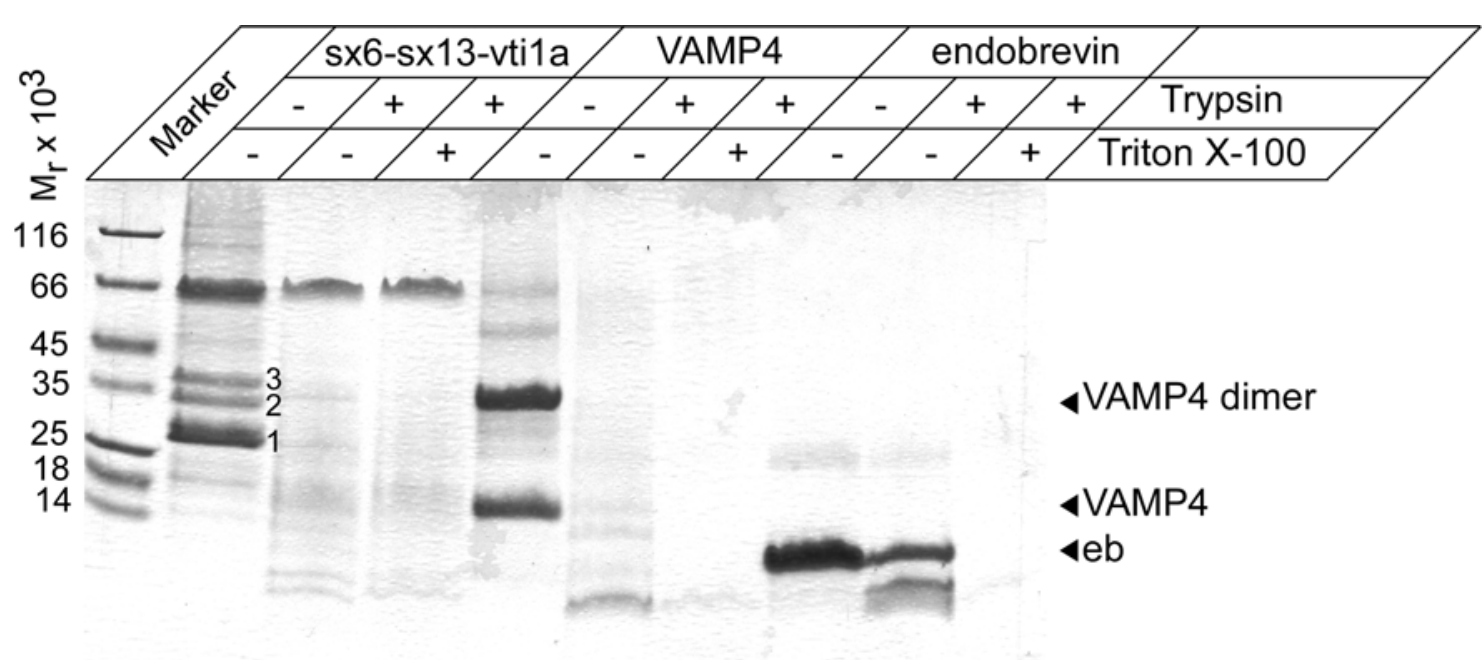

Figure 3 Trypsin Digest of Liposomes to Determine Orientation of SNAREs. $20 \mu$ l of liposomes containing sx6-sx13-vti1a, VAMP4 or endobrevin were digested by adding $5 \mu \mathrm{l} 1 \mathrm{mg} / \mathrm{ml}$ Trypsin. The mix was incubated for $1 \mathrm{~h}$ at $37^{\circ} \mathrm{C}$. Alternatively the liposomes were solubilised by adding Triton X-100 in a final concentration of $2 \%$ in addition to trypsin. The reactions were analyzed on a $10 \%$ Schaegger gel stained with Commassie Blue. The numbers indicate the early endosomal Q-SNAREs. 1, vti1a; 2, syntaxin 6; 3, syntaxin 13.

\subsection{Liposomal Fusion Assays - Fluorescence Spectrophotometry}

The liposomal dequenching assay was performed according to Struck (Struck et al., 1981). Before each measurement the cuvette (quartz cuvette $1 \mathrm{~cm}$, Hellma, Mühlheim, Germany) was soaked in $0.2 \%$ Hellmanex, then rinsed with $\mathrm{dd}_{2} \mathrm{O}$ and blow dried with a stream of $\mathrm{N}_{2}$. The fluorometer chamber was set to $37^{\circ} \mathrm{C}$. The NBD emission signal was measured by exciting at $460 \mathrm{~nm}$ and measuring emission at $538 \mathrm{~nm}$. In the experiments with Oregon green and Texas red labelled liposomes the donor signal (excitation at 490nm and emission at $520 \mathrm{~nm}$ ) and the FRET signal (excitation at $490 \mathrm{~nm}$ and emission at $615 \mathrm{~nm}$ ) were measured. The data were collected in Excel and ISA format. The liposomes were used in final concentration of either $1.5 \mu \mathrm{M}$ or $3 \mu \mathrm{M}$ per protein for the donor (Rhodamine and NBD loaded) liposomes and $3 \mu \mathrm{M}$ final protein concentration (per protein) for the acceptor liposomes in a total volume of $50 \mu$ l. In some experiments $5 \mu \mathrm{l}$ donor liposomes and $35 \mu \mathrm{l}$ acceptor liposomes were used regardless of the protein concentration and $10 \mu$ l buffer was added (final 
volume $50 \mu \mathrm{l}$ ). For the liposomes with labelled lipids $20 \mu \mathrm{l}$ donor and $20 \mu \mathrm{l}$ acceptor liposomes were used in a final volume of $50 \mu \mathrm{l}$. For the competitive inhibition the soluble SNARE was either added to the reaction mix simultaneously when the liposomes were combined or it was added to one liposome population with a $1 \mathrm{~h}$ preincubation at $37^{\circ} \mathrm{C}$. For protease digestion experiments the liposomes were incubated with $5 \mu$ l of a $1 \mathrm{mg} / \mathrm{ml}$ trypsin solution for $1 \mathrm{~h}$ at $37^{\circ} \mathrm{C}$ before starting the experiment.

\subsection{Determination of $\mathrm{IC}_{50}$ Values in Inhibition Experiments}

$I C_{50}$ is the concentration of an inhibitor at which half-maximal effect is reached. To determine the $\mathrm{IC}_{50}$ values for soluble SNARE fragments in different inhibition experiments, the percent of total dequenching at $t=10 \mathrm{~min}$ for each experiment was determined. The percent of inhibition was calculated by normalising the values to the maximum fusion and to the minimum possible value (background) that was obtained by recording background signal of empty (protein free) liposomes that were labelled with NBD and Rhodamine. These values were plotted as percent of total inhibition (y) versus the natural logarithm (In) of the concentration $(\mathrm{mol} / \mathrm{l})$ of soluble SNARE fragments $(\mathrm{x})$. This dose response curve can be fitted using a sigmoidal function $y=\left(a / 1+\exp \left(-\left(x-x_{0}\right) / b\right)\right)$. 


\section{Results}

\subsection{Biochemical Characterisation of the Early Endosomal SNARE Complex}

\subsubsection{Recombinant Proteins}

The full-length, cytosolic and the core fragments of the five SNAREs that are most likely involved in early endosomal fusion were cloned and expressed in $E$. coli as described in the Materials and Methods section. A schematic diagram of the SNARE domains is depicted in Figure 4. Two of them are $Q_{a}$-SNAREs (syntaxin 13 and syntaxin 16 ), the $Q_{b}-S N A R E$ is vti1a, the $Q_{c}-S N A R E$ syntaxin 6 , and the R-SNARE VAMP4. An overview of the different constructs that were used in this study is given in Table 2 .

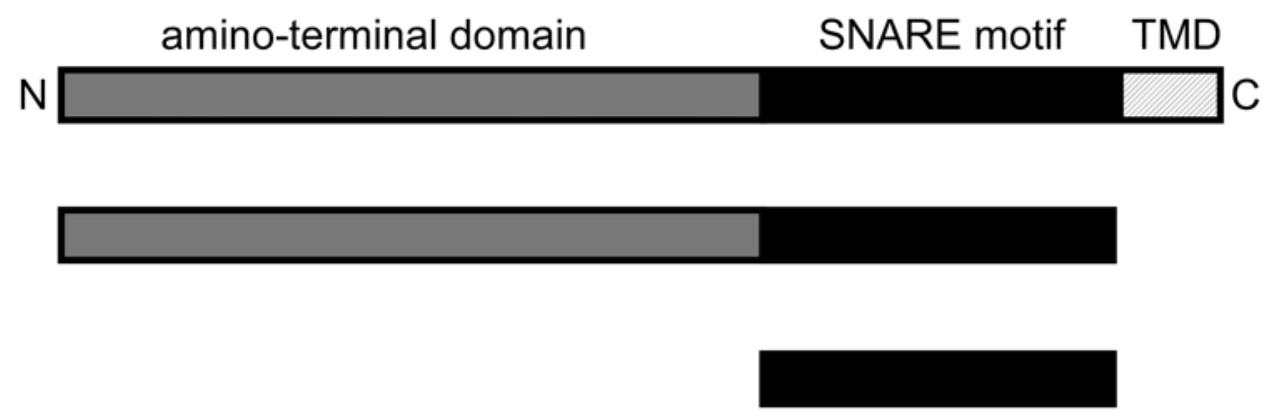

$1 \overline{0}$ aa

Figure 4 Schematic Diagram of the Different SNARE Constructs. The depicted constructs were cloned and expressed for syntaxin 6, syntaxin 13, syntaxin 16, vti1a and VAMP4. Full-length (top), cytosolic (middle) and SNARE- (also called core-) fragment (bottom), N, amino-terminal; C, carboxy-terminal domain. 


\begin{tabular}{|l|c|c|c|}
\hline Protein (species) & Fragment & Amino acids & Vector \\
\hline \multirow{3}{*}{$\begin{array}{l}\text { Syntaxin 6 } \\
\text { (homo sapiens) }\end{array}$} & Full-length & $1-255$ & $\mathrm{pET28a}$ \\
\cline { 2 - 4 } & Cytosolic & $1-234$ & $\mathrm{pGEX-KG}$ \\
\cline { 2 - 4 } & SNARE motif & $169-234$ & $\mathrm{pET28a}$ \\
\hline $\begin{array}{l}\text { Syntaxin 13 } \\
\text { (rattus norvegicus) }\end{array}$ & Full-length & $1-274$ & $\mathrm{pET28a}$ \\
\cline { 2 - 4 } & Cytosolic & $1-250$ & $\mathrm{pET28a}$ \\
\cline { 2 - 4 } & SNARE motif & $182-250$ & $\mathrm{pET28a}$ \\
\hline \multirow{3}{*}{$\begin{array}{l}\text { Syntaxin 16 } \\
\text { rattus norvegicus) }\end{array}$} & Full-length & $1-324$ & $\mathrm{pET28a}$ \\
\cline { 2 - 4 } & Cytosolic & $1-302$ & $\mathrm{pET28a}$ \\
\cline { 2 - 4 } & SNARE motif & $237-302$ & $\mathrm{pET28a}$ \\
\hline \multirow{2}{*}{\begin{tabular}{l} 
(rattus norvegicus) \\
\cline { 2 - 4 }
\end{tabular}} & Full-length & $1-217$ & $\mathrm{pET28a}$ \\
\cline { 2 - 4 } & Cytosolic & $1-192$ & $\mathrm{pET28a}$ \\
\cline { 2 - 4 } & SNARE motif & $115-192$ & $\mathrm{pET28a}$ \\
\hline $\begin{array}{l}\text { VAMP4 } \\
\text { (mus musculus) }\end{array}$ & Full-length & $1-141$ & $\mathrm{pET28a}$ \\
\cline { 2 - 4 } & Cytosolic & $1-118$ & $\mathrm{pGEX-KG}$ \\
\cline { 2 - 4 } & SNARE motif & $47-117$ & $\mathrm{pET28a}$ \\
\hline
\end{tabular}

Table 2 Overview of the Protein Constructs of Syntaxin 6, Syntaxin 13, Vti1a and VAMP4. For each construct the source species, the first and the last amino acids and the expression vector used are listed.

After expression and purification the SNARE motifs, the cytosolic fragments and the full-length proteins were checked for purity by SDS-PAGE analysis. The protein bands migrated at the appropriate positions according to the molecular weight marker and each purified protein was at least 95\% pure (Figure 5). 


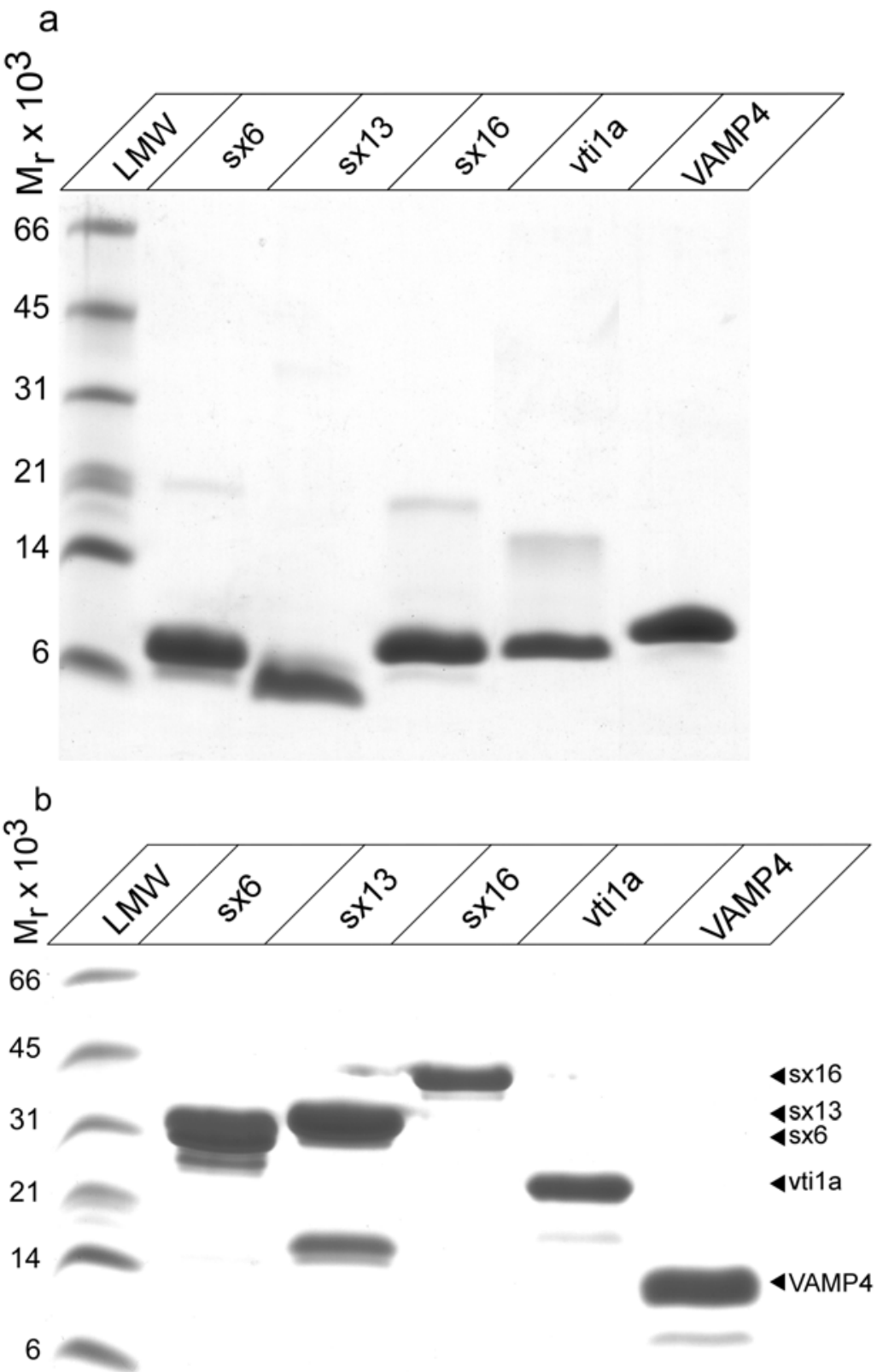




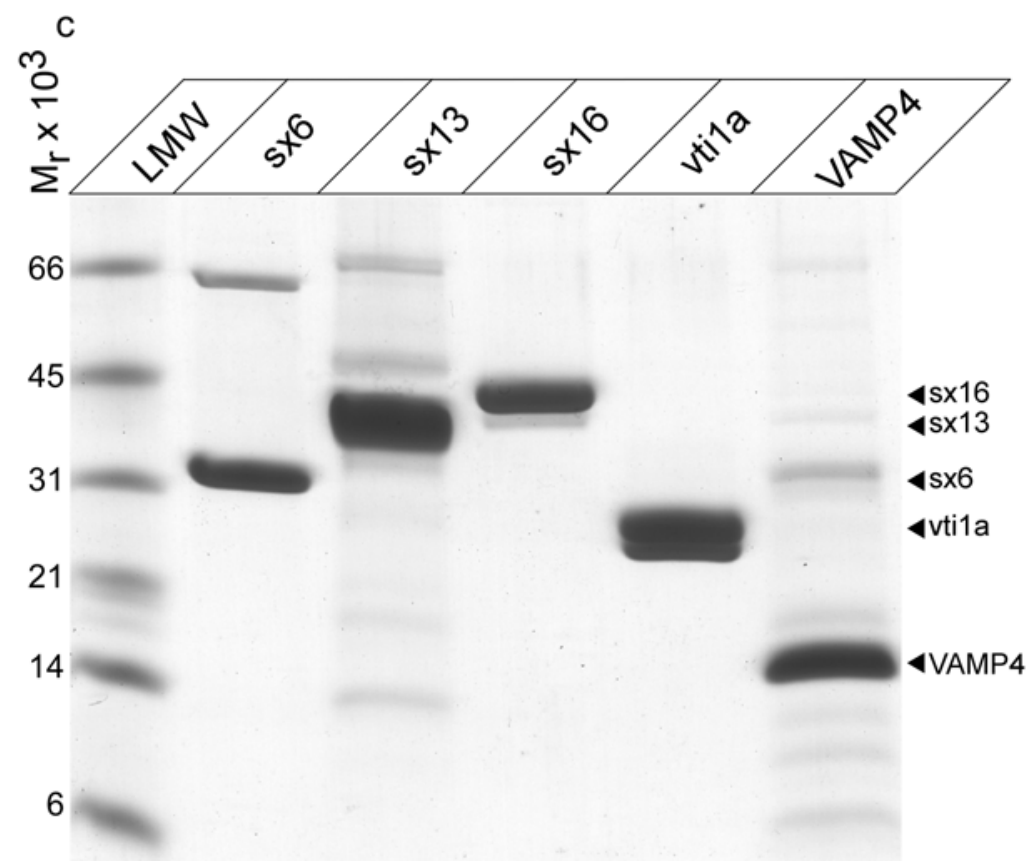

Figure 5 SDS-PAGE Analysis of the Five Recombinant (a) Core Fragments. (b) Cytosolic Fragments and (c) Full-length Constructs. A total of $5 \mu \mathrm{g}$ of each protein was loaded on a $10 \%$ Tricine SDS PAGE gel after boiling for $5 \mathrm{~min}$. The gel was stained with Commassie Blue.

\subsubsection{Characterisation of the Generated Antibodies}

As a tool to characterise the SNARE proteins, antibodies were generated that are specific for these SNAREs. The cytosolic fragments of the five SNAREs described in 3.1.1 were used as antigens to immunise rabbits. The sera of these rabbits were tested on PC12 or NRK cell blots (Figure 6). The sera show one strong signal for each antigen and thus the antibodies are suitable for further experiments. For the experiments with the early endosomal fusion assay the rabbit sera were further affinity purified using recombinant proteins coupled to $\mathrm{CNBr}$-sepharose. 


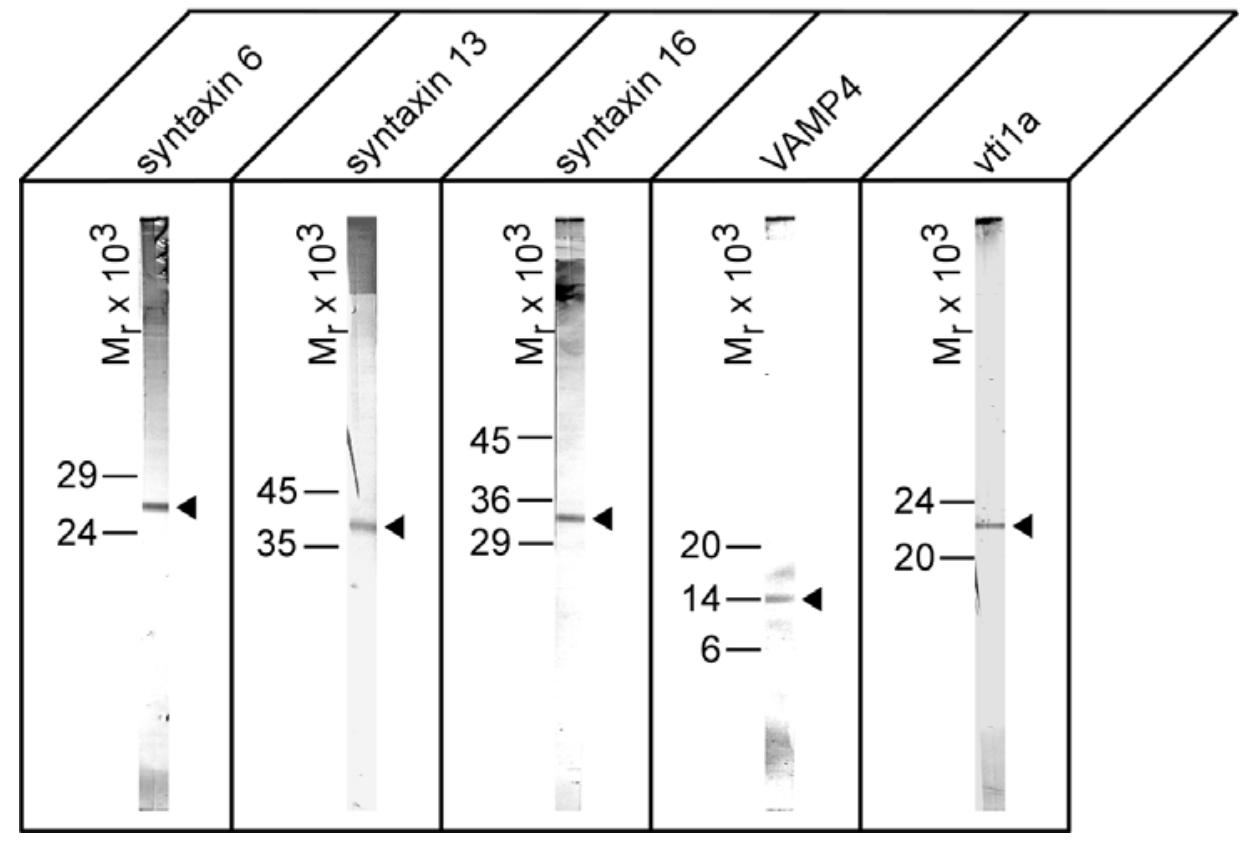

Figure 6 Antisera against Syntaxin 6, Syntaxin 13, Syntaxin 16, VAMP4 and Vti1a Show Specific Signals on NRK or PC12 Cell Blots. $150 \mu \mathrm{g}$ PC12 or NRK cell homogenate were loaded on a $10 \%$ Tricine SDS PAGE gel (broad comb) after boiling. The gel was blotted on a nitrocellulose membrane and the membrane was cut into strips. The strips were incubated with the rabbit sera sx6 1:500 (NRK), sx13 1:1000 (PC12) sx16 1:300 (NRK), VAMP4 1:300 (NRK) and vti1a 1:100 (PC12) for 1h at RT. A goat antimouse antibody linked to alkaline phosphatase was used as secondary antibody 1:5000 $1 \mathrm{~h}$ at RT. Some blots showed background generated by unspecific colour reactions of the developing solutions.

\subsubsection{Characterisation of the Function of the Recombinant SNAREs and the IgGs in the Early Endosomal Fusion Assay}

The recombinant SNARE proteins and the affinity purified IgGs were used to inhibit the fusion of early endosomes in a newly developed in vitro fusion assay (Brandhorst PhD thesis, 2004). This work was performed in collaboration with D. Brandhorst and S.Rizzoli (MPI-bpc, Göttingen) who performed the fusion assay. The fusion assay is based on labelling different sets of PC12 endosomes with fluorescent dyes by fluid uptake. The endosomes are allowed to fuse in the presence of rat brain cytosol and an ATP regenerating system. Fusion is measured by determination of colocalisation of the endosomes on cover slips by fluorescence microscopy (Brandhorst PhD thesis, 2004). 
Antibodies have been shown to block fusion if the SNARE they are targeted against is involved in the respective fusion step (Antonin et al., 2000b; Mills et al., 2001). To investigate the role of the different SNAREs in early endosomal fusion, $F_{a b}$-fragments directed against the SNAREs were used to inhibit the fusion. The $F_{a b}$-fragments were used at a final concentration of $4 \mu \mathrm{M}$. An inhibition of $70 \%$ was observed with $F_{a b}$-fragments against $s \times 6$, an inhibition of $60 \%$ was observed with $\mathrm{F}_{\mathrm{ab}}$-fragments against sx13 and vti1a and an inhibition of $40 \%$ with $\mathrm{F}_{\mathrm{ab}}$-fragments against VAMP4 (Figure 7 ). In addition, $\mathrm{F}_{\mathrm{ab}}$-fragments against the late endosomal sx8, the neuronal R-SNARE synaptobrevin and against synaptophysin, were used in the assay. While the $F_{a b}$-fragments against sx8 and synaptobrevin had no effect, the ones against synaptophysin had an inhibitory effect and inhibited by $60 \%$. This was surprising. Although synaptophysin is enriched on early endosomes it does not take part in the fusion reaction of early endosomes, it only travels through them, in the course of vesicle recycling (Holroyd et al., 1999). Since early endosomes are heavily loaded with synaptophysin, steric hindrance effects of the $F_{a b}$-fragments might have given false results. In light of these findings, $F_{a b}$-fragments may not be reliable tools in the characterisation of functional SNARE proteins in fusion reactions. 


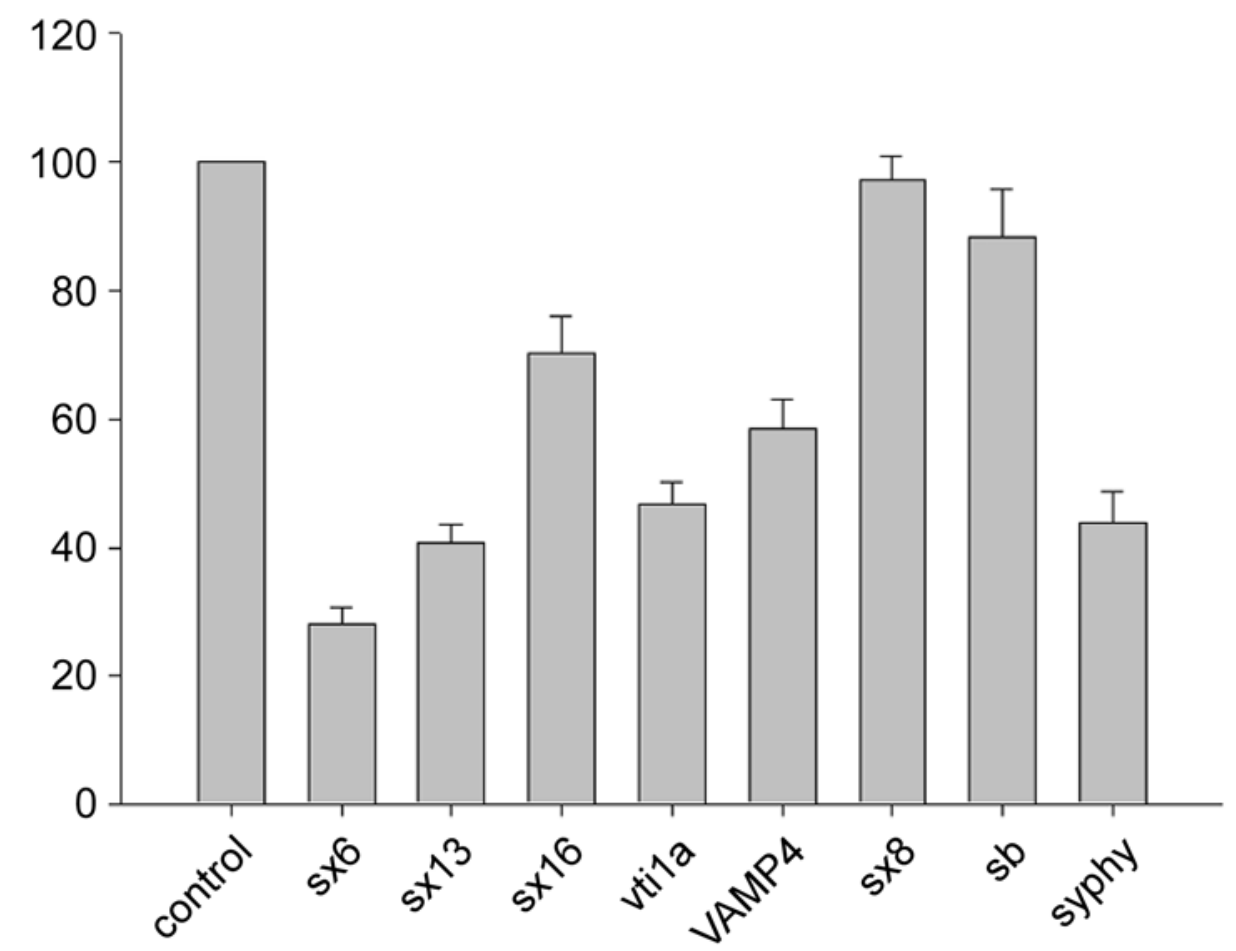

Figure 7 Inhibition of Early Endosomal Fusion using $F_{a b}-$ fragments Against Different Proteins. IgGs were affinity purified and digested in order to obtain $\mathrm{F}_{\mathrm{ab}}$ - fragments. These were used to inhibit the fusion reaction in a concentration of $4 \mu \mathrm{M}$. sx8, syntaxin 8; sb, synaptobrevin; syphy, synaptophysin ( $F_{a b}$-fragments generated by D. Brandhorst, fusion assay performed by D. Brandhorst).

In order to properly investigate the SNAREs that promote the fusion of early endosomes, the soluble fragments of the five candidates were added to the reaction as competitive inhibitors. They are thought to block fusion, because the endogenous SNAREs on the endosomes are driven into mixed cis-complexes and are not available for the formation of functional trans-complexes with cognate SNARE proteins that reside on other endosomes. Using single SNAREs or triple combinations as inhibitors may result in varying potencies depending on the protein and the combination that was used. Inhibition depends on the ability of soluble protein(s) to interact with the complementary wild type SNAREs. Depending on the combination of proteins and their inhibitory effects a conclusion about available SNAREs on the endosomal surface can be made. 
The results of all inhibition experiments are shown in Figure 8. At this stage it was not yet clear, which of the two $Q_{a}$-SNAREs, syntaxin 13 or syntaxin 16 , would be functioning in this fusion reaction. Figure 8a shows the effect of the candidate early endosomal Q-SNAREs including syntaxin 16 and syntaxin 13 . The Q-SNAREs syntaxin 6, syntaxin 13 , syntaxin 16 and vti1a were tested singly and in combination. Inhibition of fusion was observed in all cases. However, syntaxin 13 was more potent ( $60 \%$ inhibition) than syntaxin 16 , which only resulted in moderate inhibition ( $\sim 30 \%$ inhibition). The combination of syntaxin 13 and syntaxin 16 did not exceed the inhibition observed with syntaxin 13 alone, demonstrating that the two $Q_{\mathrm{a}}$-SNAREs probably do not operate in parallel in different SNARE complexes. Vti1a and syntaxin 6 also caused partial inhibition ( $20 \%$ and $\sim 50 \%$ inhibition, respectively). However, the triple combination of syntaxin 13, syntaxin 6 and vti1a resulted in almost complete inhibition. In contrast, the triple combination including syntaxin 16 did not exhibit such strong inhibition ( $\sim 30 \%$ inhibition), suggesting that syntaxin 16 does not interact functionally with vti1a and syntaxin 6 .

No inhibition was observed when the neuronal Q-SNAREs were added at the same concentration, regardless of whether single SNAREs, or a combination of all Q-SNAREs, were used. Similarly, the late endosomal Q-SNAREs, singly or in combination, were largely ineffective in inhibiting fusion (Figure 8b).

This set of data clearly indicated that syntaxin 13, vti1a and syntaxin 6 are the three Q-SNAREs involved in early endosomal homotypic fusion. To identify the R-SNARE candidate of the complex, the R-SNAREs VAMP4, synaptobrevin, endobrevin, cellubrevin, Ti-VAMP, VAMP5, ykt6 and sec22 were used in the fusion reaction as competitive inhibitors (Figure 8c) (fusion assay with $\mathrm{R}$ SNARE inhibition performed by Silvio Rizzoli). Only VAMP4 inhibits the reaction potently with more than $40 \%$ inhibition. A slight inhibition was also observed for endobrevin supporting earlier findings (Antonin et al., 2000b). VAMP4 thus has similar inhibitory effects on the fusion as the three candidate Q-SNAREs alone. If fusion of early endosomes required for example the R-SNARE to be present on one side and the three Q-SNAREs to be present on the other side of the fusing membranes then the addition of the soluble R-SNARE would be 
expected to result in much stronger inhibition than was observed. The same applied to the Q-SNAREs when they were applied singly. Most endosomes contain all four SNAREs (Brandhorst PhD thesis, 2004), therefore, it is likely that the SNAREs are equally distributed over the early endosomal membranes and that more than one arrangement of SNAREs is able to mediate fusion.

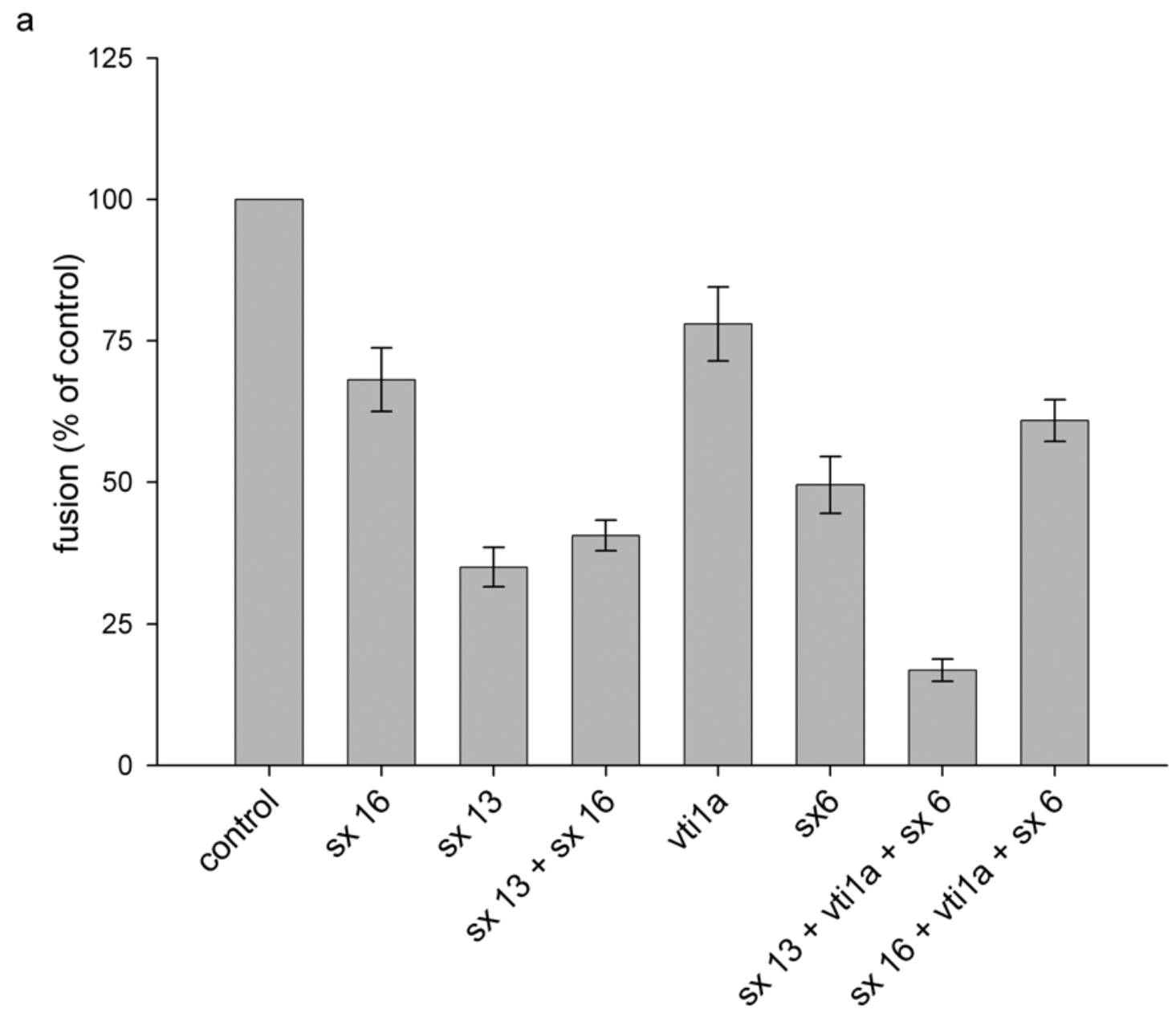




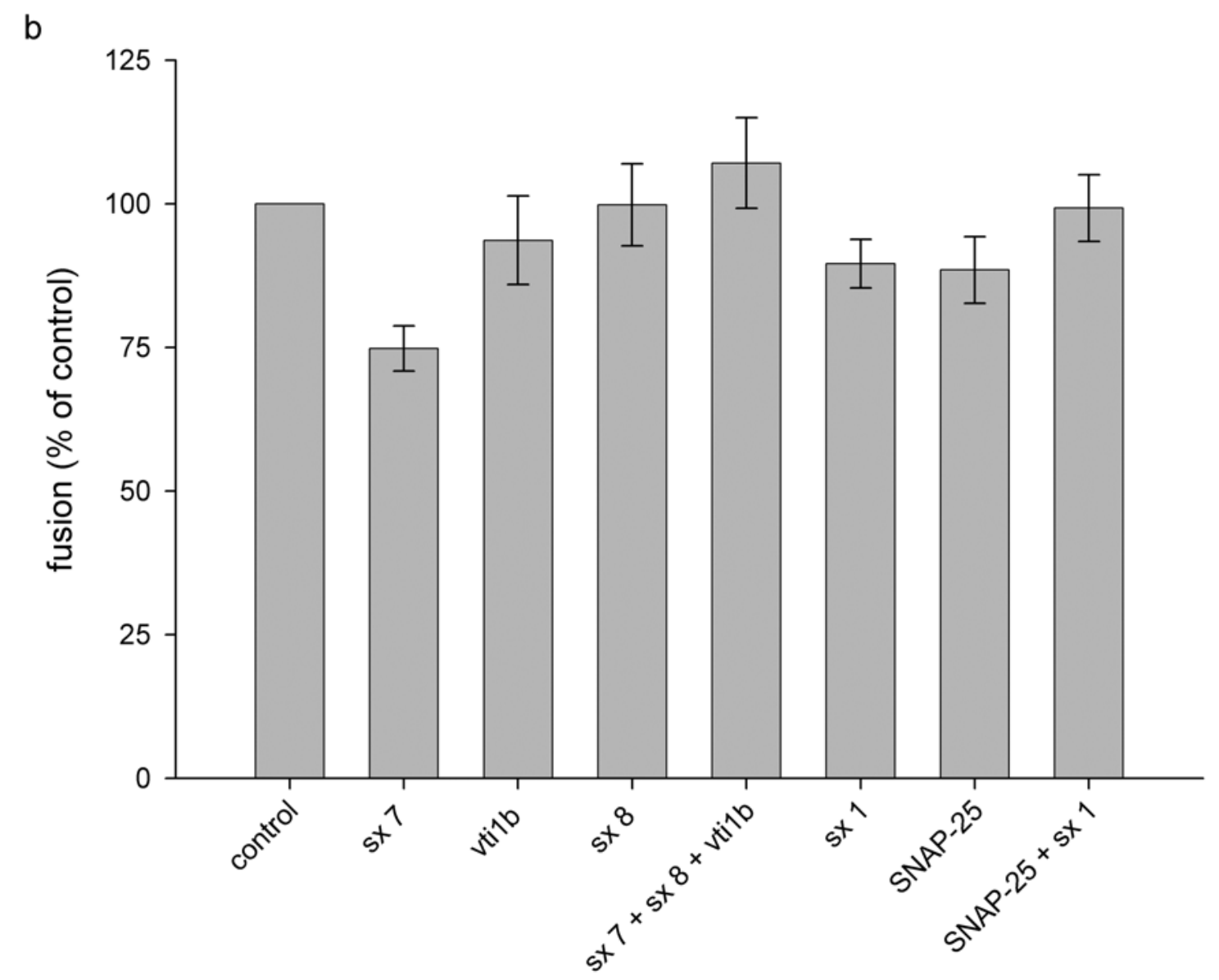




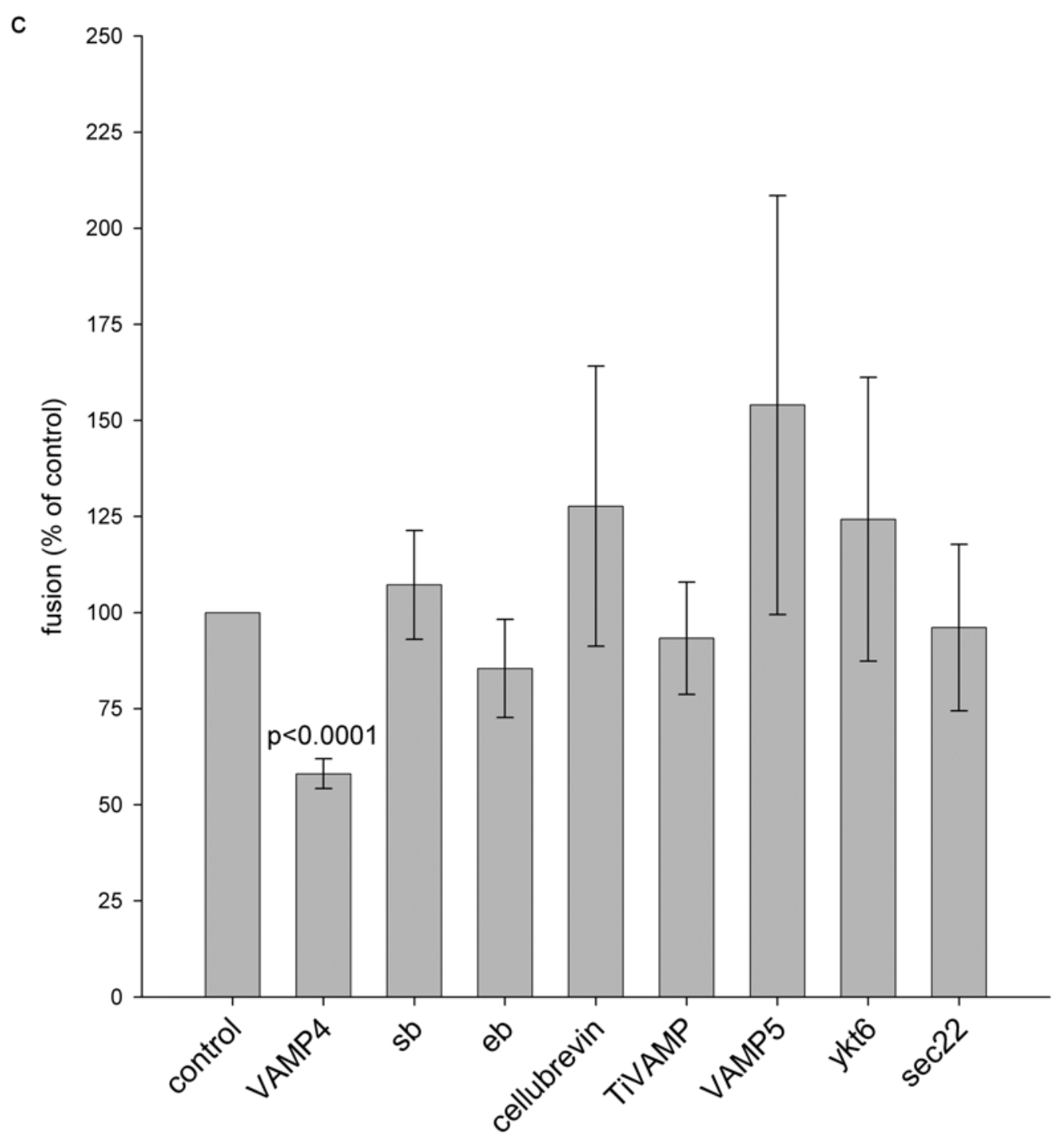

Figure 8 Effects of Soluble Q- and R-SNAREs on Early Endosomal Fusion. a) Effects of Q-SNAREs considered to be involved in the fusion of early endosomes, including syntaxin 16 (sx 16), syntaxin 13 (sx 13), vti 1a, syntaxin 6 (sx 6). Values are means \pm SEM of 7 to 10 independent experiments. b) Effects of soluble fragments of late endosomal and neuronal Q-SNAREs including syntaxin 7 (sx 7), vti 1b, syntaxin 8 (sx 8), syntaxin 1 (sx 1) and SNAP-25. Values are means \pm SEM of 7 to 13 independent experiments. c) Effects of soluble R-SNARE fragments including VAMP4, synaptobrevin/VAMP2 (syb), endobrevin/VAMP8 (eb), cellubrevin/ VAMP3, TiVAMP/VAMP7, VAMP5. Values are means \pm SEM of 3 to 7 independent experiments. Individual Q-SNAREs were used at $12 \mu \mathrm{M}$, with R-SNAREs tested at $25 \mu \mathrm{M}$. Fusion assays performed by D. Brandhorst and S. Rizzoli. 


\subsubsection{Optimisation of the Assembly Conditions}

The soluble fragments of the Q-SNAREs sx6, sx13 and vti1a, and of the RSNARE VAMP4 and the $F_{a b}$-fragments against these proteins exhibited the most potent inhibition in the early endosomal fusion assay. It was therefore assumed that these are the members of the early endosomal SNARE complex. The next step was to test if these four proteins assemble into a SNARE complex that can be purified and characterised. In order to identify the optimal assembly conditions for the complex, the four SNAREs motifs of syntaxin 6 , syntaxin 13, vti1a and VAMP4 were mixed in equimolar ratios in different concentrations of $\mathrm{NaCl}$ or urea and circular dichroism (CD) spectroscopy was performed. With CD spectroscopy the a-helicity of SNARE complexes can be determined (Fasshauer et al., 1997). It is known that the SNARE monomers exhibit almost no ellipticity, indicating that they are largely unstructured. A massive increase of ellipticity is observed when the coiled coil structure forms. Therefore, CD spectroscopy can be used to follow SNARE complex formation.

First we screened for optimal conditions for complex formation. Initial experiments revealed that a SNARE complex consisting of syntaxin 6 , syntaxin 13, vti1a and VAMP4 was not soluble in standard buffers. Different concentrations of salt and urea were used to investigate optimal conditions for the formation of this complex. The four proteins were mixed in buffers containing between $300 \mathrm{mM}$ and $1000 \mathrm{mM} \mathrm{NaCl}$ and $2 \mathrm{M}$ or $4 \mathrm{M}$ urea.

The early endosomal SNARE core fragments were incubated for $16 \mathrm{~h}$ at $4^{\circ} \mathrm{C}$. CD spectroscopy was performed and the a-helicity of the assembly mixes was used as the readout for complex formation. The a-helicity in the incubated sample varied depending on different assembly conditions (Figure 9a). The monomers were largely unstructured and all of the assembly condition exhibited more ellipticity than the theoretical sum of the monomers (Figure 9b). Assembly in the presence of $1000 \mathrm{mM} \mathrm{NaCl}$, however, led to the largest increase of ellipticity. 


\section{Results}

a

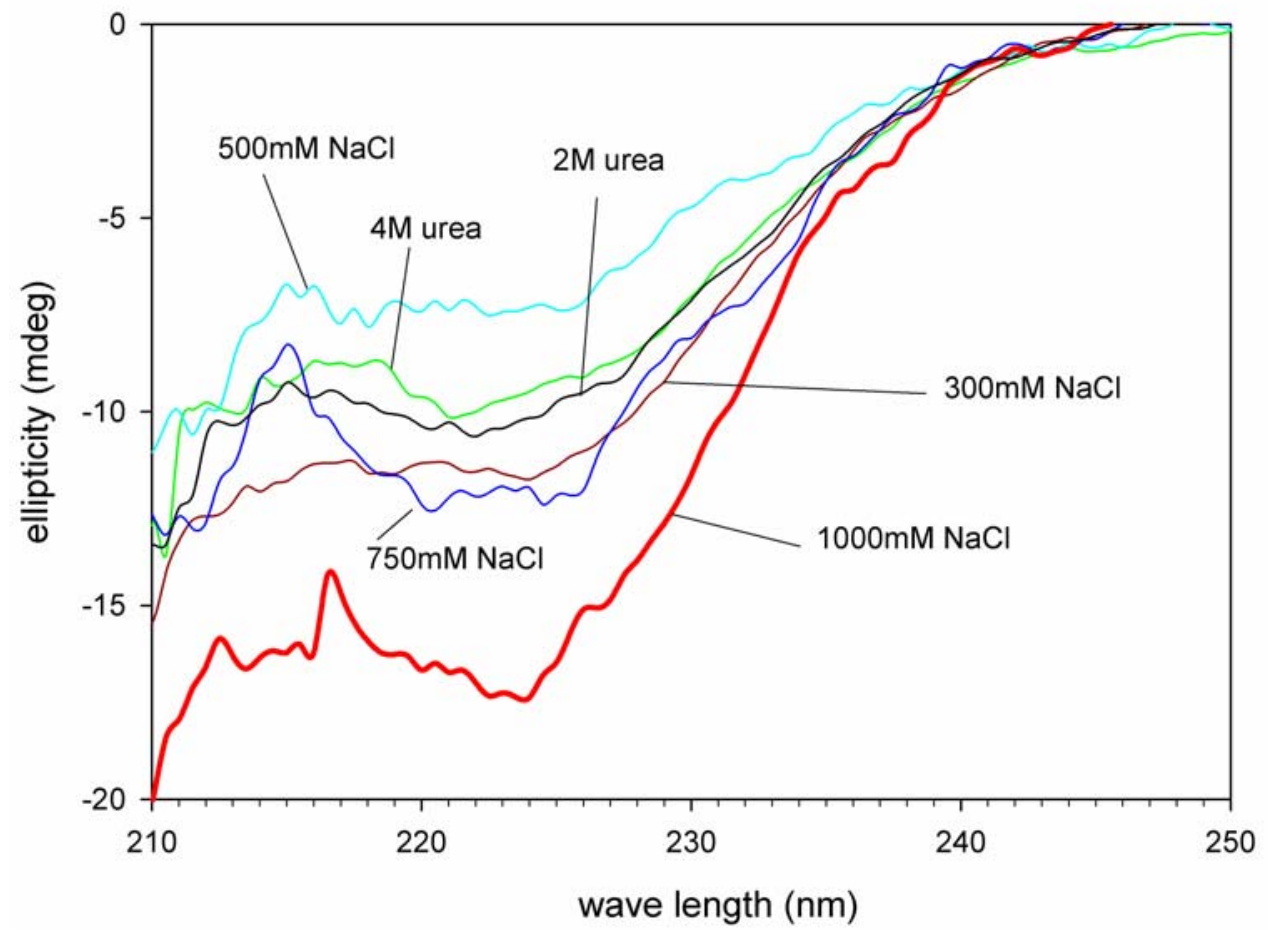




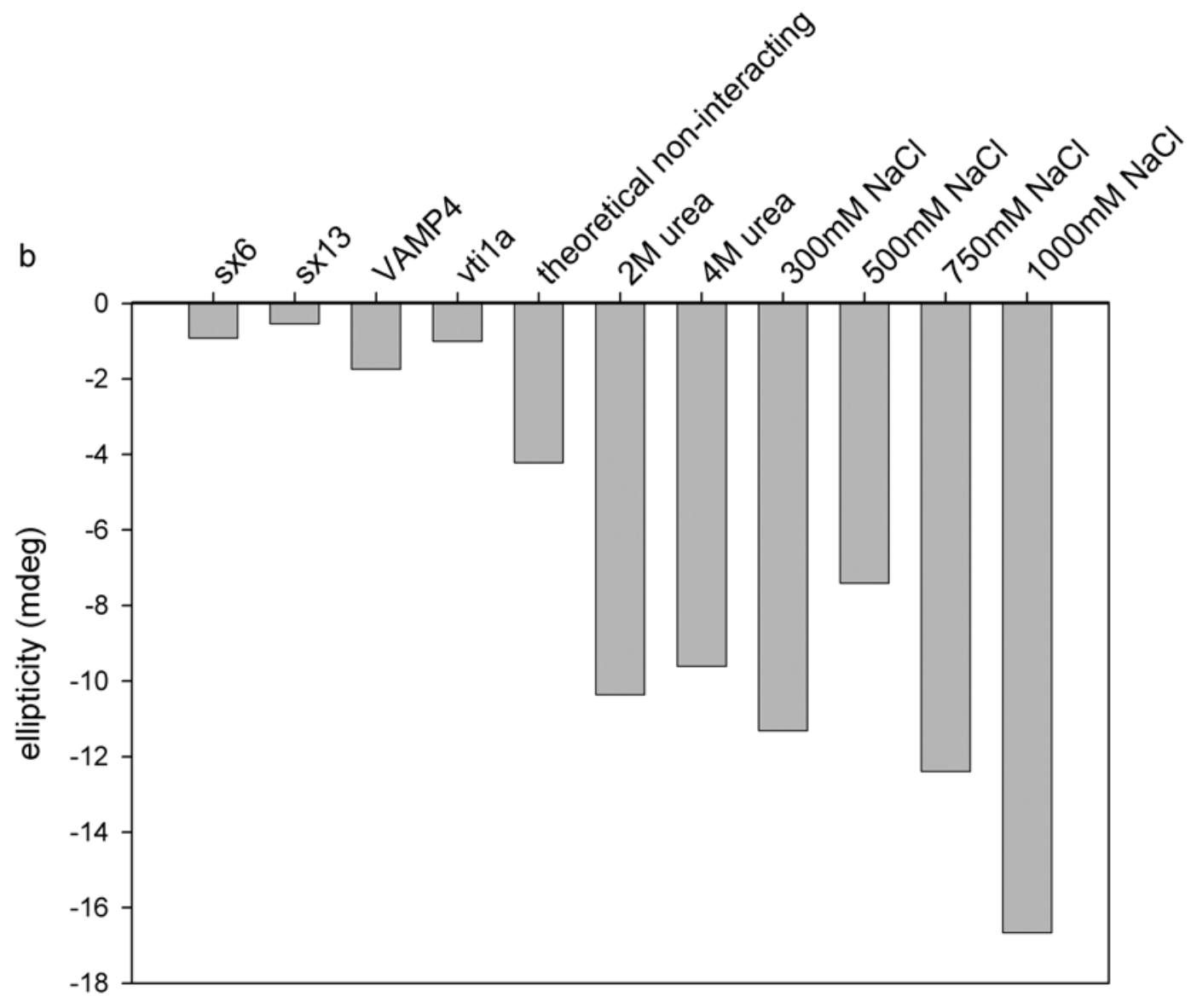

Figure 9 Analysis of Different Assembly Conditions using CD Spectroscopy. The SNARE motifs of sx6, sx13, vti1a and VAMP4 were mixed in equimolar amounts $(5 \mu \mathrm{M}$ each) and incubated over night at $4^{\circ} \mathrm{C}$ in $\mathrm{Tris} / \mathrm{HCl}$ buffer, $\mathrm{pH} 7.4$ with different concentrations of salt or urea. a) The CD spectra of the different complex mixes are shown as ellipticity (mdeg) at the respective wavelength. The characteristic minima of $\alpha$-helices are at $222 \mathrm{~nm}$ and $208 \mathrm{~nm}$. Urea exhibits high absorbance of UV light, thus spectra below $210 \mathrm{~nm}$ could not be obtained. b) The minima of the graphs at $222 \mathrm{~nm}$ shown in a bar diagram. The monomers of the four SNAREs were measured and the sum of their signals is taken as the signal in absence of measurable interaction.

In order to determine the formation of partial complexes, all possible double and triple combinations using the four early endosomal SNAREs were investigated. No combination other than the 1:1:1:1 of all four SNAREs led to an increase of ellipticity above the respective theoretical sum of the monomers. Thus, one can conclude that there were no 1:1:2 or 2:2 complexes with a helical structure, like the neuronal 2:1-complex incorporating syntaxin 1 and SNAP25 (Fasshauer et al., 1997). Unlike in the neuronal SNARE complex, no change in a-helicity is observed when 1:1 or 1:1:1 combinations of the late endosomal SNAREs are 
incubated (Antonin et al., 2000a), probably indicating that no partial helical complexes form.

\subsubsection{Assembly and Purification of the New SNARE Complex}

In the previous experiment it was shown that complex assembly was more efficient in buffers containing $1 \mathrm{M} \mathrm{NaCl}$. These conditions were chosen for an upscale assembly. The complex was generated in larger quantities in order to characterise well known features of SNARE complexes like SDS resistance or thermostability and to screen for crystallisation conditions. The four SNARE core fragments of sx6, sx13, vti1a and VAMP4 were mixed in equimolar ratios using approximately $5 \mathrm{mg}$ per protein and dialyzed $(20 \mathrm{mM}$ Tris/ $\mathrm{HCl}$ buffer with $1 \mathrm{mM}$ EDTA, $1 \mathrm{mM}$ DTT and $1000 \mathrm{mM} \mathrm{NaCl}$ ) for $16 \mathrm{~h}$ at $4^{\circ} \mathrm{C}$. In order to separate the complex from monomers ion-exchange chromatography was performed. For this, the assembly mix was dialyzed to a low salt buffer that enables binding to a MonoQ ion-exchange column. In studies with the neuronal and the late endosomal complex it was shown that concentrations of urea lower than 5-8M do not harm the complex. The synaptic complex is not unfolded in buffers containing up to $8 \mathrm{M}$ urea (Fasshauer et al., 2002) suggesting that a concentration of $1 \mathrm{M}$ urea could be used to purify the early endosomal complex, thus urea was added to prevent precipitation. The dialysis buffer contained $20 \mathrm{mM}$ Tris/ $/ \mathrm{HCl}, 100 \mathrm{mM} \mathrm{NaCl}$ and $1 \mathrm{M}$ urea. After loading of the complex to a MonoQ ion-exchange column it was eluted using a linear gradient of increasing salt concentration by mixing buffer $\mathrm{A}(20 \mathrm{mM}$ Tris/ $\mathrm{HCl}, \mathrm{pH} 7.4,0 \mathrm{mM} \mathrm{NaCl}, 1 \mathrm{mM}$ EDTA, 1mM DTT) with buffer $B$ (buffer $A$ with $1000 \mathrm{mM} \mathrm{NaCl}$ ) (Figure 10). The fractions 7 to 9 were analysed on a $10 \%$ Tricine SDS gel with Coomassie Blue staining (Figure 11). 


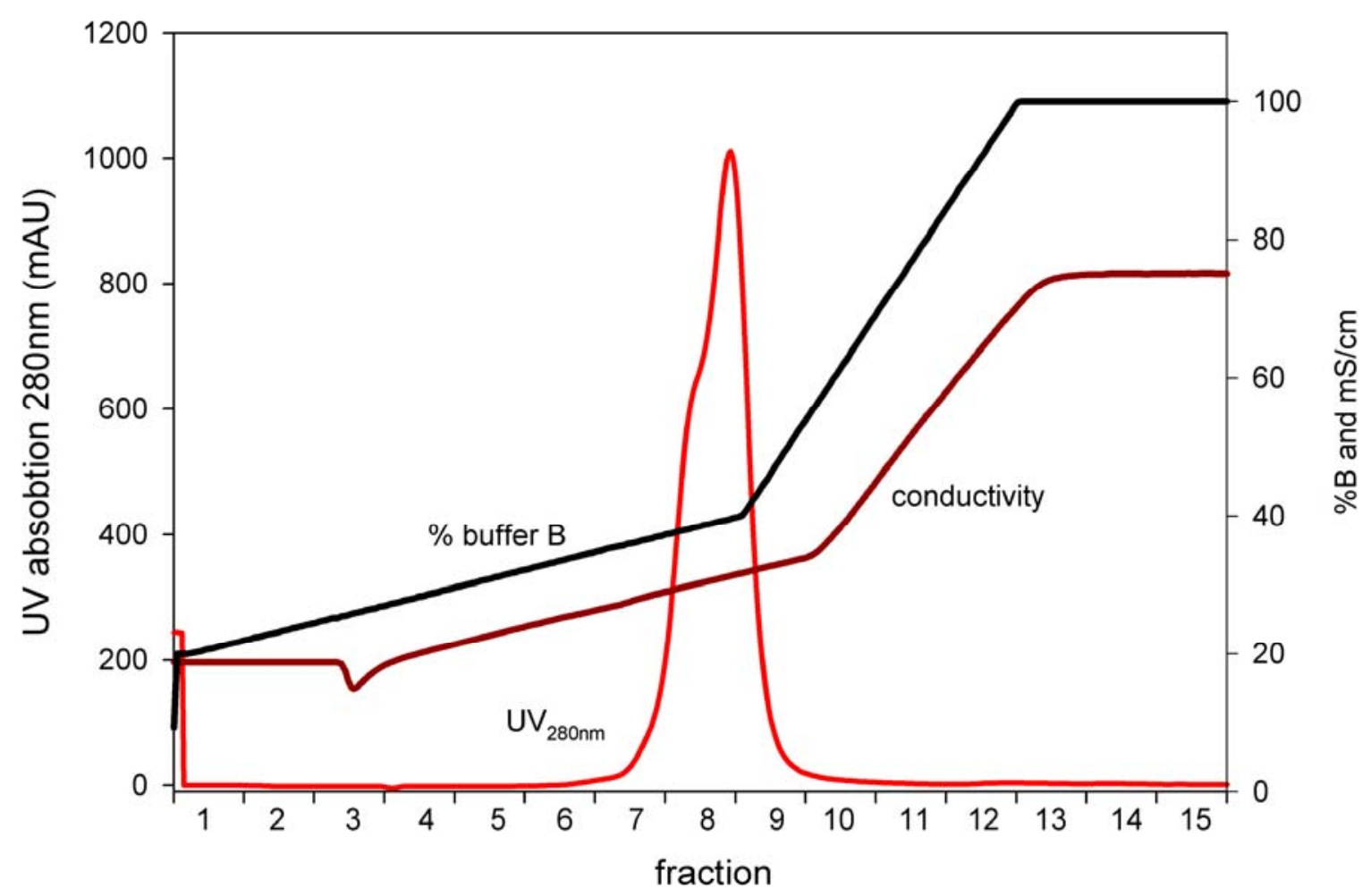

Figure 10 Purification of the Early Endosomal SNARE Complex. Elution profile of the complex in ion-exchange chromatography. The left axis and the red line show the UV absorption at $280 \mathrm{~nm}$ in $\mathrm{mAU}$, the right axis shows the conductivity in $\mathrm{mS} / \mathrm{cm}$ and $\%$ buffer $B$ (buffer $\mathrm{B}, 1000 \mathrm{mM} \mathrm{NaCl}$ ). The black line indicates the programmed salt gradient and the brown line the actual conductivity. Fractions of $2 \mathrm{ml}$ were collected.

SDS-resistance is observed for the neuronal (Fasshauer et al., 1997) and late endosomal SNARE complexes (Antonin et al., 2000a). The early endosomal complex migrated as a single band in SDS-PAGE between 35 and $45 \mathrm{kDa}$ (Figure 11) thus also exhibiting SDS-resistance. Oligomers of the complex were not observed. However, the early endosomal complex is not completely SDS resistant as monomers were visible on the bottom of the gel. The complex eluted at higher salt concentrations $(\mathrm{NaCl}>500 \mathrm{mM})$ (Figure 10). The Q-SNARE monomers usually eluted earlier than that $(\mathrm{NaCl}<500 \mathrm{mM})$ and thus an overlap of the elution of non-incorporated monomers with the elution of the complex can be excluded. The VAMP4 core fragment did not bind to the MonoQ column. However, VAMP4 monomers were still visible at the bottom of the gel (Figure 11), thus VAMP4 seen in fractions 7,8 and 9 were derived from the complex. 
Two SNARE core fragments, i.e. syntaxin 6 and vti1a, migrated at the same height on the gel. Additional analysis was performed by Dr. Henning Urlaub (MPI-bpc, Mass spectrometry). Using mass spectrometry the four SNAREs in the complex band and syntaxin 6 and vti1a in the overlapping monomer bands were identified. After boiling, the complex band disappeared and only monomers were visible on the SDS gel (Figure 11 a). These experiments demonstrated that the early endosomal complex containing syntaxin 6 , syntaxin 13, vti1a and VAMP4 assembled in high salt conditions and remained soluble in low salt buffer as long as urea was present. The complex migrated as one band on SDS-PAGE and was partially SDS resistant.

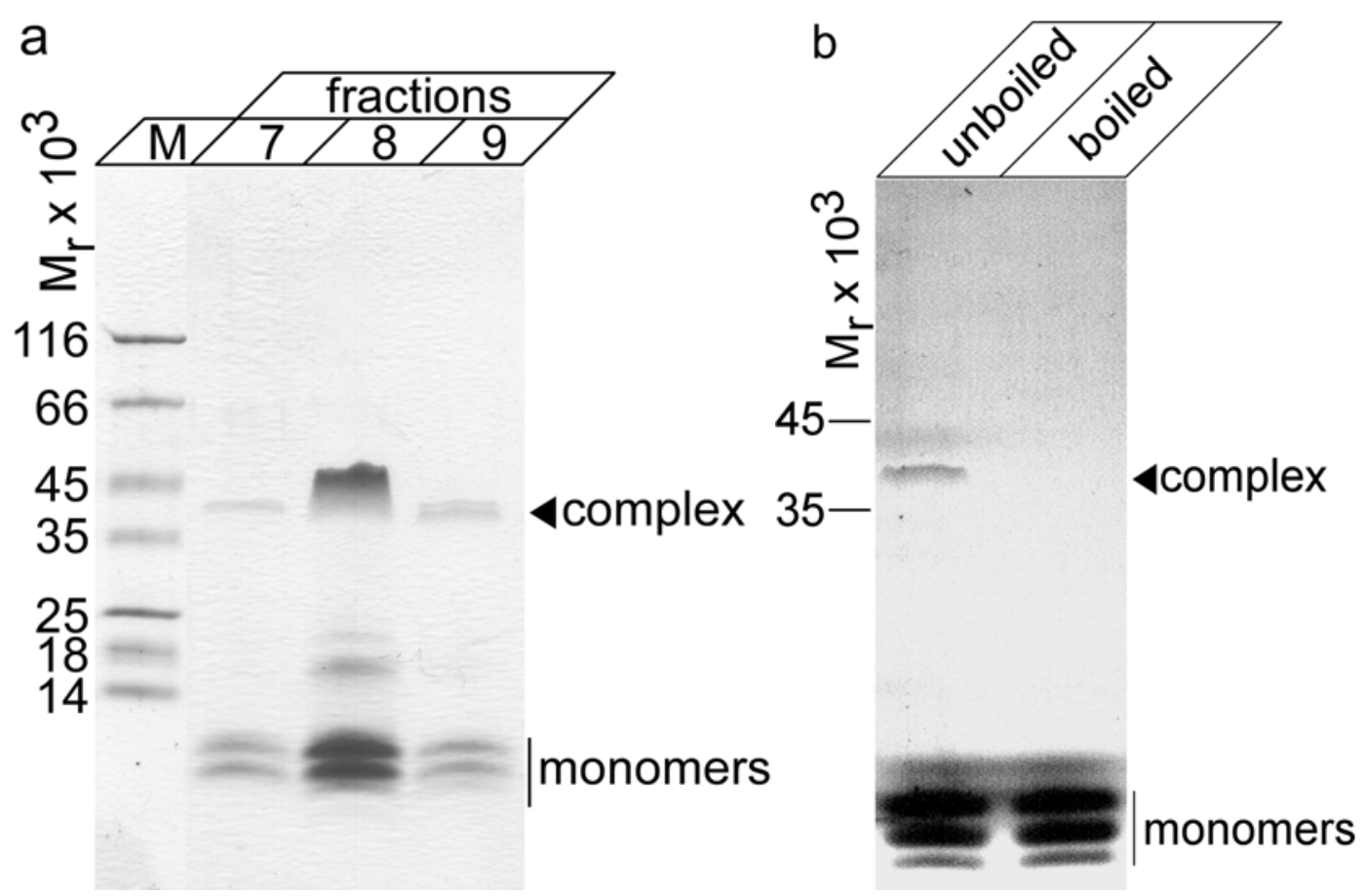

Figure 11 Analysis of Fractions 7-9 by SDS-PAGE and Coomassie Blue Staining. $10 \mu \mathrm{l}$ of fractions 7,8 and 9 from the chromatography shown in Figure 10 were loaded on a $10 \%$ Tricine SDS gel. a) The SDS resistant complex migrates between 35 and $45 \mathrm{kDa}$. b) The complex is not fully SDS resistant as indicated by the monomers below the complex bands. The complex band disappears after boiling. 


\subsubsection{Molecular Mass Determination using Multi Angle Laser Light Scattering}

The previous experiments showed that the complex migrated at the appropriate position in SDS PAGE and that it contains the four different SNARE fragments. The theoretical molecular mass for the complex containing the four monomers in a 1:1:1:1 ratio is $36.5 \mathrm{kDa}$. To verify a $1: 1: 1: 1$ composition the exact molecular mass of the complex had to be determined using multi angle laser light scattering (MALLS). With MALLS the molecular mass of molecules can be determined. This was done by first separating the molecules by gel filtration according to their size followed by the determination of the molecular mass by measuring light scattering. After assembly and purification by ion-exchange chromatography the complex was dialysed to $20 \mathrm{mM}$ Tris $/ \mathrm{HCl}$ buffer, $\mathrm{pH} 7.4$ with $2 \mathrm{M}$ urea and either $500 \mathrm{mM}$ or $1000 \mathrm{mM} \mathrm{NaCl}$. The complex eluted as a single peak with a molecular mass of $38(+/-1.1) \mathrm{kDa}$ in $1 \mathrm{M}$ salt condition (Figure 12a) thus confirming the predicted composition. It is known from the late endosomal and the neuronal complex that SNARE complexes tend to oligomerise in low salt conditions (Antonin et al., 2002b; Fasshauer et al., 1997). Oligomerisation was observed for the early endosomal SNARE complex at lower salt concentrations. In the buffer containing $500 \mathrm{mM} \mathrm{NaCl}$ the molecular mass shifted to $60+/-0.7 \mathrm{kDa}$ which could be an indication for complex oligomerisation/dimerisation (Figure 12b). 

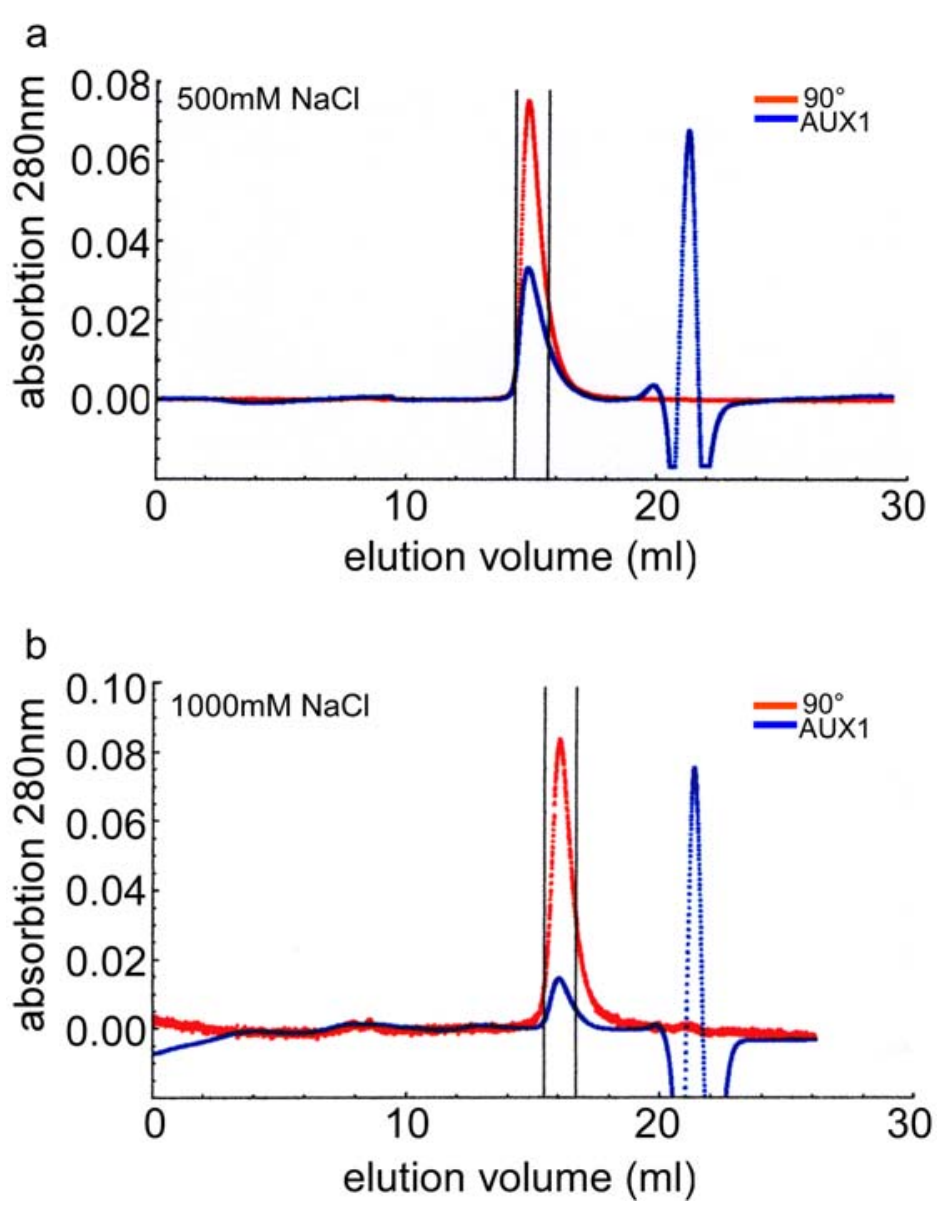

Figure 12 Determination of the Molecular Mass of the Early Endosomal SNARE Complex. The complex was gel filtrated and the molecular mass was measured by MALLS. a) The experiments were performed either in $500 \mathrm{mM} \mathrm{NaCl}$ buffer or b) $1000 \mathrm{mM} \mathrm{NaCl}$ buffer. A shift of the peaks to the right was observed when comparing a.) with b.) indicating an increase in molecular mass. The calculated mass for $1000 \mathrm{mM}$ buffer is $38 \mathrm{kDa}$ and for the $500 \mathrm{mM}$ buffer $60 \mathrm{kDa}$ suggesting oligomerisation. The molecular mass was calculated using the ASTRA software after defining the elution peaks with the vertical borders. UV230, absorbance at $230 \mathrm{~nm} ; 90^{\circ}$, light scattering detector in right angle to laser.

\subsubsection{The Early Endosomal SNARE Complex Exhibits Unfolding-Refolding Hysteresis as Determined by CD Spectroscopy}

SNARE complexes are thermostable, and fall apart at high temperatures. Unfolding can be monitored by circular dichroism spectroscopy $(C D)$ because the ellipticity decreases upon denaturation of the complex. SNARE complexes usually unfold between $80^{\circ} \mathrm{C}$ and $90^{\circ} \mathrm{C}$. After reducing the temperature, the 
complexes start to refold only at much lower temperatures (between $35-50^{\circ} \mathrm{C}$ ). This effect hysteresis is a feature of SNARE complexes (Fasshauer et al., 2002). In order to investigate if the new SNARE complex exhibits such behaviour, the unfolding and refolding curve was monitored using $C D$ spectroscopy (Figure 13).

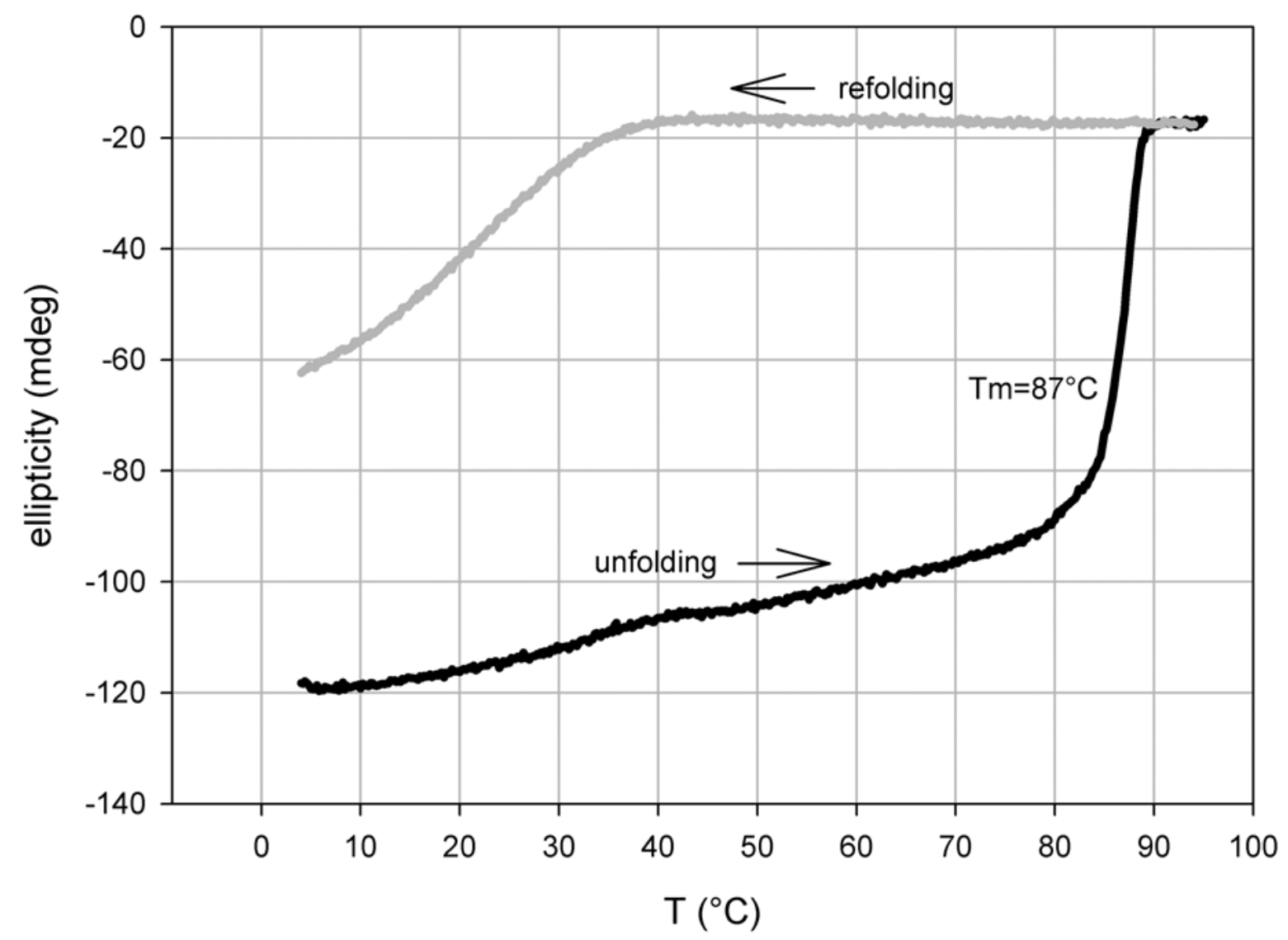

Figure 13 Unfolding and Refolding Transitions of the Early Endosomal SNARE Complex Exhibit Hysteresis. Thermal unfolding and reconstitution of the early endosomal SNARE complex $(15 \mu \mathrm{M}$ protein in $20 \mathrm{mM}$ Tris/ $\mathrm{HCl}, 1000 \mathrm{mM} \mathrm{NaCl}, 1 \mathrm{mM}$ EDTA, $1 \mathrm{mM}$ DTT, $0.2 \mathrm{M}$ urea) monitored by $\mathrm{CD}$ spectroscopy at $222 \mathrm{~nm}$ by increasing the temperature from $4^{\circ} \mathrm{C}$ to $94^{\circ} \mathrm{C}$ with $60^{\circ} \mathrm{C} / \mathrm{h}$. Unfolding occurred in a steep transition with $\mathrm{T}_{\mathrm{m}}=87^{\circ} \mathrm{C}$ (black line). To monitor refolding the temperature was lowered from $90^{\circ} \mathrm{C}$ to $4^{\circ} \mathrm{C}$ at a rate of $70^{\circ} \mathrm{C} / \mathrm{h}$. Refolding occurred only partially.

The ellipticity of the complex in a final concentration of $15 \mu \mathrm{M}$ was monitored at $222 \mathrm{~nm}$ at $4^{\circ} \mathrm{C}$ and the temperature was then increased at a constant rate to $95^{\circ} \mathrm{C}$. Upon heating, the ellipticity decreased slowly until $80^{\circ} \mathrm{C}$ was reached. The complex unfolded completely at $90^{\circ} \mathrm{C}$. Upon lowering the temperature the $C D$ signal stayed constant at -18 mdeg until the temperature reached approximately $35^{\circ} \mathrm{C}$. The complex then started to refold. This experiment 
demonstrates clearly that the early endosomal complex exhibits hysteresis. As the complex had not fully refolded upon reaching $4^{\circ} \mathrm{C}$ the question was whether complete refolding is possible. To answer this question the temperature was set to $4^{\circ} \mathrm{C}$ and the complex was allowed to assemble over several hours. At the beginning of this experiment the ellipticity was about $-60 \mathrm{mdeg}$. The ellipticity reached approximately -100 mdeg after 16 hours at $4^{\circ} \mathrm{C}$, thus showing almost complete reassembly. This experiment also demonstrated that the assembly kinetic was slower as compared to the neuronal SNARE complex (Fasshauer et al., 1999).

\subsubsection{Disassembly of the Complex Using NSF and $\alpha$-SNAP}

SNARE complexes are disassembled by the ATPase NSF and its co-chaperone a-SNAP. In order to investigate whether NSF and $\alpha$-SNAP are able to disassemble the early endosomal SNARE complex the following experiment was performed: the early endosomal SNARE complex was incubated with NSF, a-SNAP, ATP and either $\mathrm{MgCl}_{2}$ or EDTA (Figure 14). Different salt concentrations $(500 \mathrm{mM} / 1000 \mathrm{mM})$ were used in order to check for possible inhibition of the reaction by higher salt concentrations $(1 \mathrm{M} \mathrm{NaCl})$. In the presence of ATP and $\mathrm{Mg}^{2+}$ and $0.5 \mathrm{M} \mathrm{NaCl}$ the complex band disappeared almost completely; if EDTA was added, the complex stayed intact. Thus, NSF and $\alpha-S N A P$ are able to disassemble the early endosomal SNARE complex. 


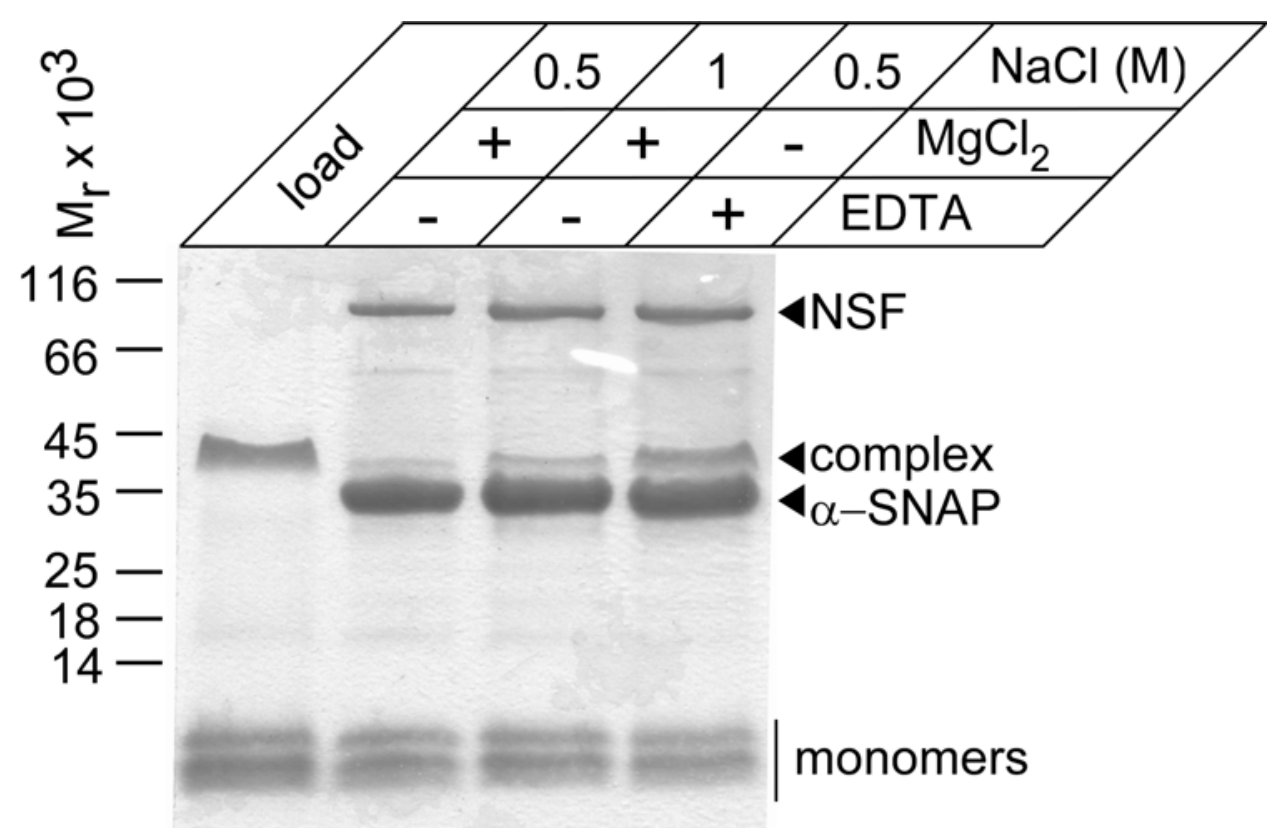

Figure 14 The Early Endosomal Complex is Dissociated by NSF and $\alpha-$ SNAP. The early endosomal SNARE complex $(2.5 \mu \mathrm{M}$ final concentration) was incubated with NSF $(2 \mu \mathrm{M})$, a-SNAP $(35 \mu \mathrm{M})$, ATP $(2.5 \mathrm{mM})$ and either $\mathrm{MgCl}_{2}(2 \mathrm{mM})$ or EDTA $(1 \mathrm{mM})$ as indicated. The dissociation of the complex by NSF and $\alpha$-SNAP was monitored by the disappearance of the complex band. The reactions were analysed by SDS-PAGE (10\% Tricine) and Coomassie Blue staining. 


\subsection{Crystal Structure of the Early Endosomal SNARE Complex}

\subsubsection{Overall Structure of the Complex}

The crystal structures of the neuronal and the late endosomal SNARE complexes have been described in detail (Antonin et al., 2002b; Sutton et al., 1998). Despite limited sequence homology among the proteins, these two complexes are remarkably similar in their overall structure as well as the alignment of their helices and the layer structure. Several surface interactions are conserved among the two complexes (Antonin et al., 2002b). The crystal structure of the early endosomal SNARE complex was solved with a resolution of 2.7A (M.Wahl, MPI-bpc). The early endosomal SNARE complex is a four helix bundle with a left handed twist and an overall rod-like structure. The positioning of the $R$ - and $Q_{a b c}-S N A R E s$ (chain $A, B, C, D$ ) in the complex is identical to the other complexes (Figure 15). Analysis of the new SNARE complex supported the notion that the structure of SNARE complexes is evolutionary well conserved.

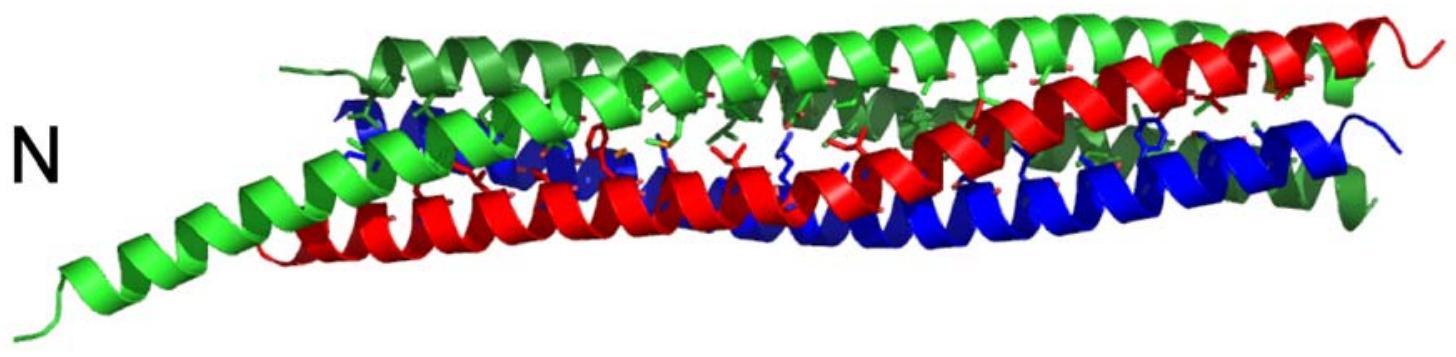

Figure 15 Overall Structure of the Early Endosomal SNARE Complex. Schematic view of the early endosomal core complex. VAMP4, blue; Syntaxin 13, red; vti1a, light green; sx6, dark green. $\mathrm{N}$, amino-terminus; C, carboxy-terminus. The amino acids participating in layer formation are depicted as sticks.

The overall structure is remarkably similar to the neuronal and late endosomal complex, as shown by a superimposition with the neuronal complex shows (Figure 16a). 


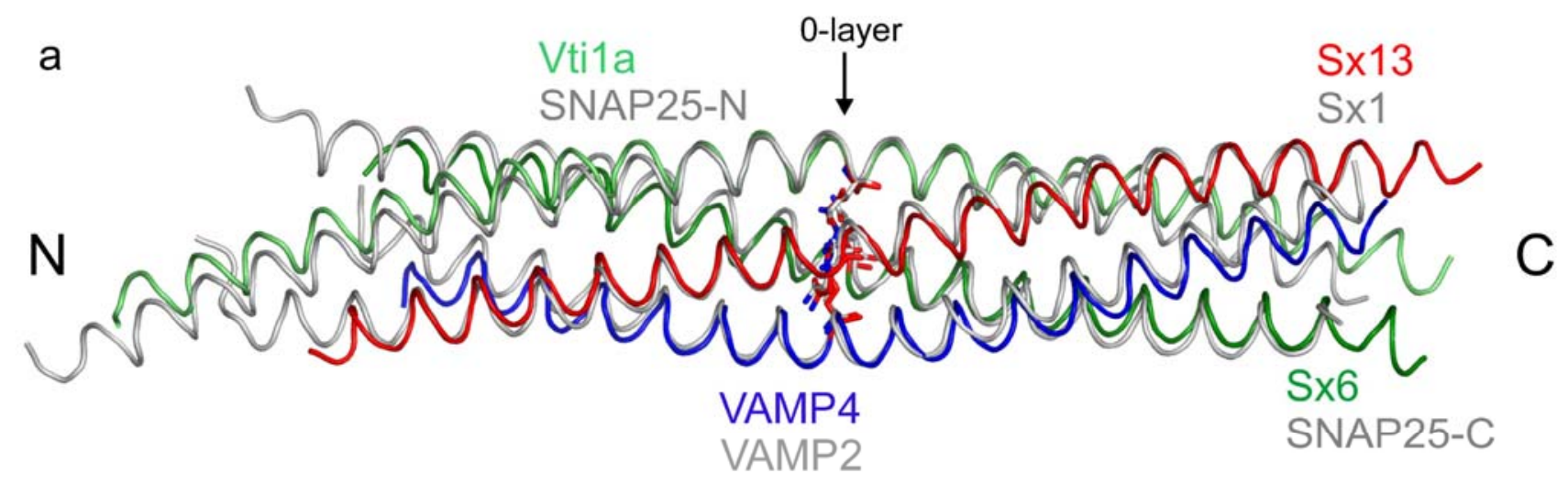

b Vti1a SNAP25-N

Vti1a

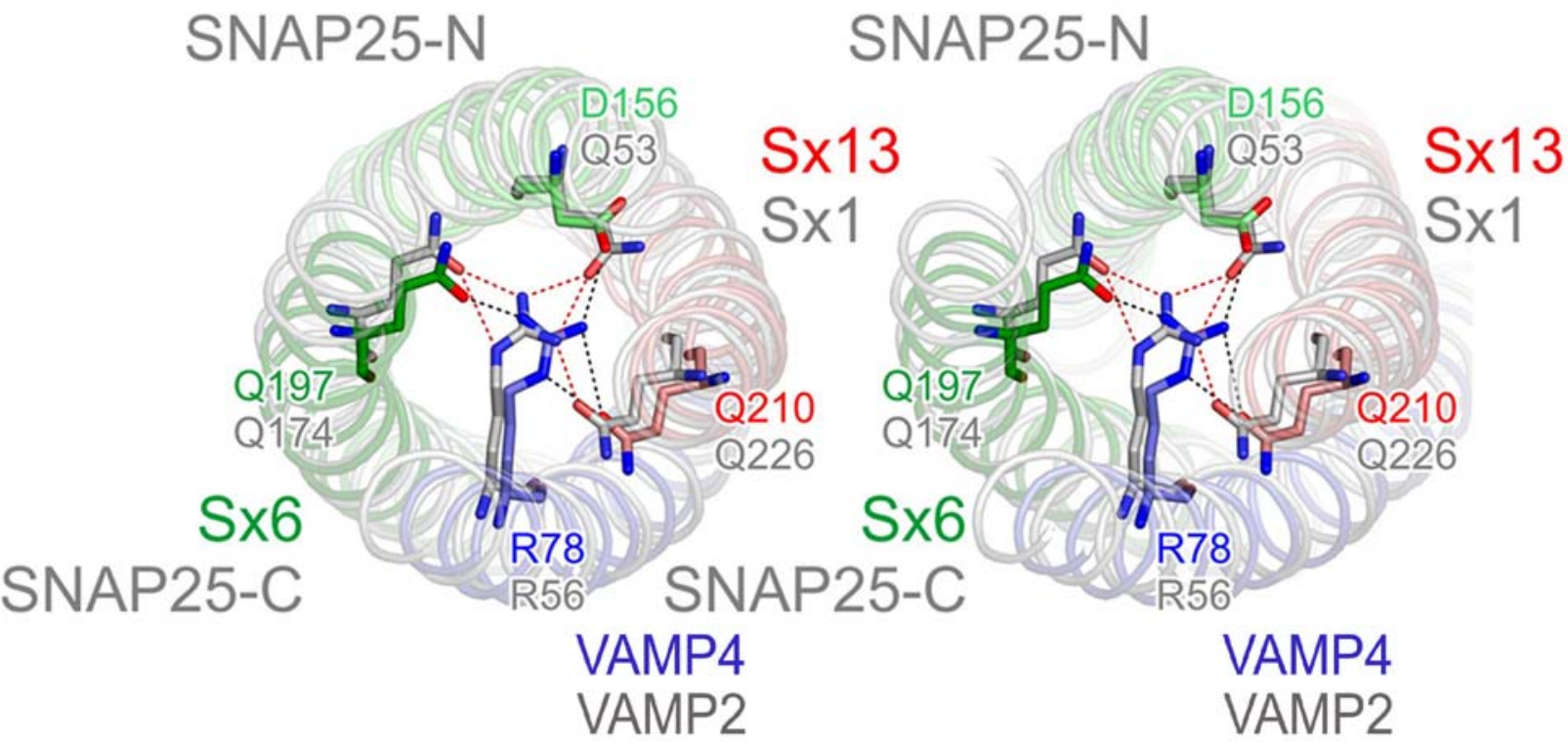

Figure 16 Structure of the Early Endosomal Complex is Highly Similar to the Neuronal Complex. a) Overlay of the early endosomal complex (in colour) and the neuronal complex (grey). b) Stereo view of the 0-layer with the unusual aspartate in vti1a, neuronal complex in grey and early endosomal complex in colour.

\subsubsection{Structure of the Individual Layers}

Compared with the other complexes, the hydrophobic layers of the early endosomal complex are very similar. Out of 64 amino acids the early endosomal and late endosomal complex have 42 identical and 15 conserved 
residues, and compared with the neuronal complex the early endosomal complex shares 31 identical and 19 conserved residues (Figure 17). In certain layers smaller amino acids are packed with bulky amino acids. Layers -3 and +6 and in addition layer +2 exhibit 'asymmetric complementarity' in the early endosomal SNARE complex. These layers may ensure the correct parallel alignment of the SNAREs when assembling to a complex (Oakley and Kim, 1998). Similar asymmetry is observed in the late endosomal complex for layers -3 and +6 (Antonin et al., 2002b) in the neuronal complex for layers $-3,-2$ and +6 (Fasshauer et al., 1998b).

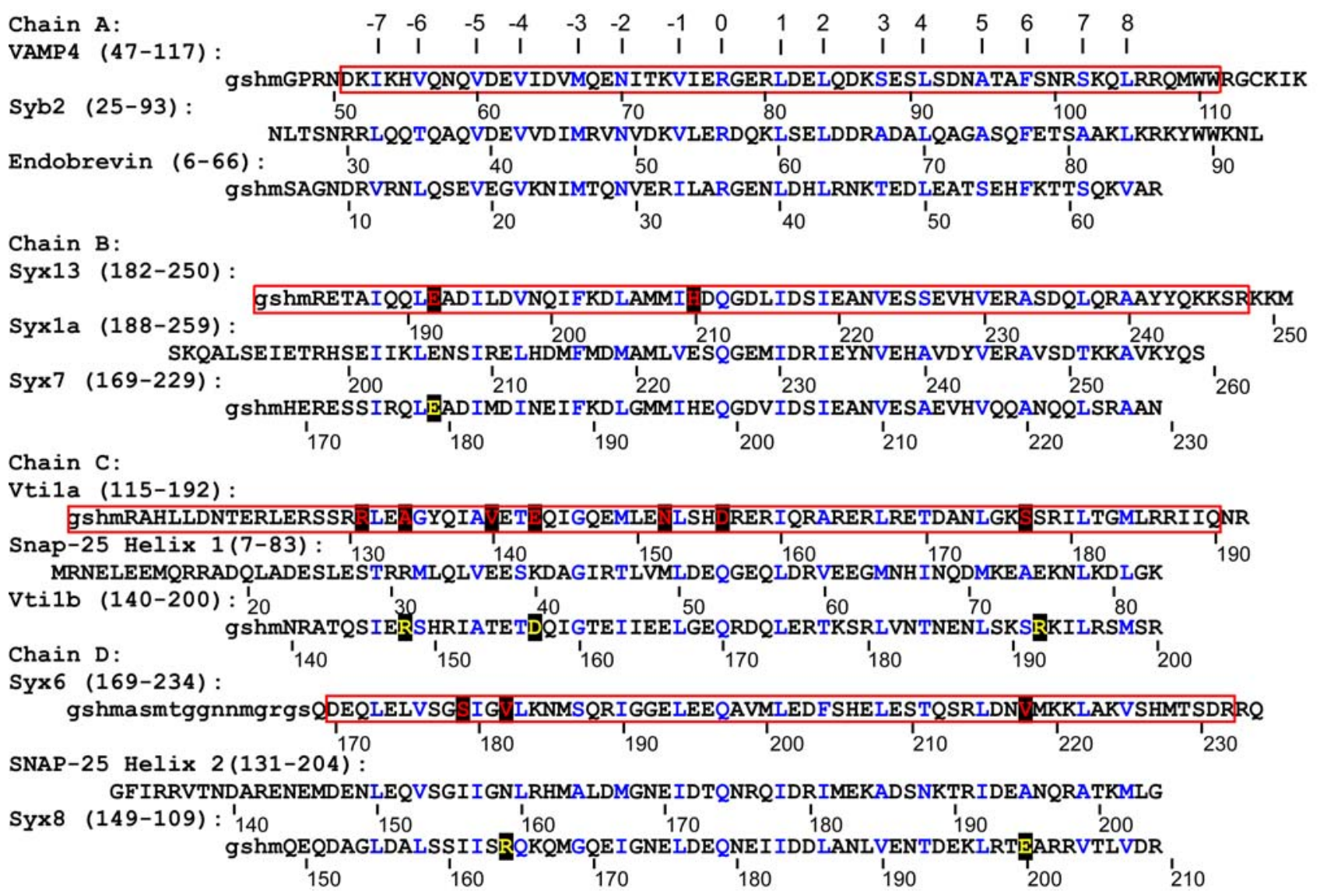

Figure 17 Three SNARE Complexes in Sequence Comparison. Sequence alignment of the $R$ - and $Q_{a b c}$ - SNAREs (chain $A, B, C, D$ ) of the two SNARE complexes with known crystal structures (the neuronal and the late endosomal) and the early endosomal SNARE complex. The alignment is restricted to the 7 layers upstream (layers -1 to -7 ) and 8 layers downstream (layers +1 to +8 ) of the ionic 0-layer. The 16 layers are shown in blue, unusual amino acids and the ones discussed in the text are shown in yellow and the red boxes indicate the amino acids that are part of the crystal structure. 


\subsubsection{Intra- and Intermolecular Surface Interactions}

The early endosomal complex exhibits a variety of inter- and intra-molecular surface interactions that are probably responsible for the thermal stability (Table 2). Most of them are hydrogen bonds and ionic interactions with one exception, which is a hydrophobic interaction. Some of these surface interactions are conserved among the neuronal and late endosomal complex (Antonin et al., 2002b) but not in the early endosomal complex. Instead, alternative interactions were found that may further stabilise the complex intermolecularly. Like in the neuronal complex only two interactions originate from the R-SNARE to the syntaxin $(\mathrm{Qa})$ as well as five interactions between $R$-chain and the $\mathrm{Q}_{\mathrm{c}}$-chain. While 10 interactions were found among the Q-SNAREs in the early endosomal complex, 17 interactions were found in the neuronal complex among the $\mathrm{Q}$ SNAREs (Sutton et al., 1998). 


\begin{tabular}{|c|c|c|c|}
\hline Intramolecular & \multicolumn{3}{|c|}{ Intermolecular } \\
\hline VAMP4-VAMP4 & VAMP4-sx6 & VAMP4-vti1a & VAMP4-sx13 \\
\hline $\begin{array}{l}\text { E80 - R81 - E84 } \\
\text { S100 - K104 } \\
\text { Q86 - E90 }\end{array}$ & $\begin{array}{l}\text { Q60 - S177 } \\
\text { K74 - E195 (A) } \\
\text { K88 - S205/E209 } \\
\text { N95 - Q212 } \\
\text { Q109 - S226/S230 }\end{array}$ & - & $\begin{array}{l}\text { S89 - N222 } \\
\text { R107 - Q236 }\end{array}$ \\
\hline sx13-sx13 & sx13-sx6 & sx13-vti1a & \\
\hline Q200 - D204 & - & $\begin{array}{l}\text { E192 - R131 (A) } \\
\text { N199 -T142 } \\
\text { K203 - E141 (C) } \\
\text { H210 - N152- vti1a156 } \\
\text { E220 - R163 (C) } \\
\text { E224/E227 - R166(C) } \\
\text { Q238 - K176 }\end{array}$ & \\
\hline vti1a-vti1a & vti1a-sx6 & & \\
\hline $\begin{array}{l}\text { R157 - Q161 } \\
\text { D156-N152 (- } \\
\left.\text { sx13 }^{\mathrm{H} 210}\right)\end{array}$ & $\begin{array}{l}\text { (E143 - S179+V140 } \\
\text { - V182) hydrophobic } \\
\text { sandwich } \\
\text { R157 - E193/E196 (C) } \\
\text { D171 - T211/R214 }\end{array}$ & & \\
\hline $\begin{array}{r}\text { sx6-sx6 } \\
\text { E193 - Q198 } \\
\end{array}$ & & & \\
\hline
\end{tabular}

Table 3 Intra- and Intermolecular Interactions in the Early Endosomal SNARE Complex. The amino acids and their position in the corresponding protein are stated. Interactions followed by (C) are conserved in the neuronal, late endosomal and early endosomal SNARE complex. Interactions followed by (A) are conserved in the neuronal and late endosomal SNARE complex and have an alternative stabilising mechanism in the early endosomal complex.

\subsubsection{0-layer}

The 0-layer in the early endosomal complex is different from the 0-layers in the other SNARE complexes (Figure 16b). Usually 3 glutamine residues and one arginine are found in the 0-layer. Here the $\mathrm{Q}_{b}$-chain vti1a contributes an aspartate (D) instead of the otherwise highly conserved glutamine (Q). This aspartate is conserved throughout the vti1a orthologues in vertebrates. The aspartate not only forms a salt bridges with the other amino acids in the 0-layer but it also interacts with vti1 $a^{\mathrm{N} 152}$. Vti1 $\mathrm{a}^{\mathrm{N} 152}$ in addition, has a salt bridge with $\mathrm{sx} 13^{\mathrm{H} 210}$ (Figure 18). A similar interaction involving residues from the 0-layer was found in the neuronal complex including the arginine-glutamine-aspartate 
interaction (synaptobrevin ${ }^{\mathrm{R} 56}-\mathrm{SNAP} 25^{\mathrm{Q} 174}-\mathrm{SNAP} 25^{\mathrm{E} 170}$ ) (Ernst and Brunger, 2003).

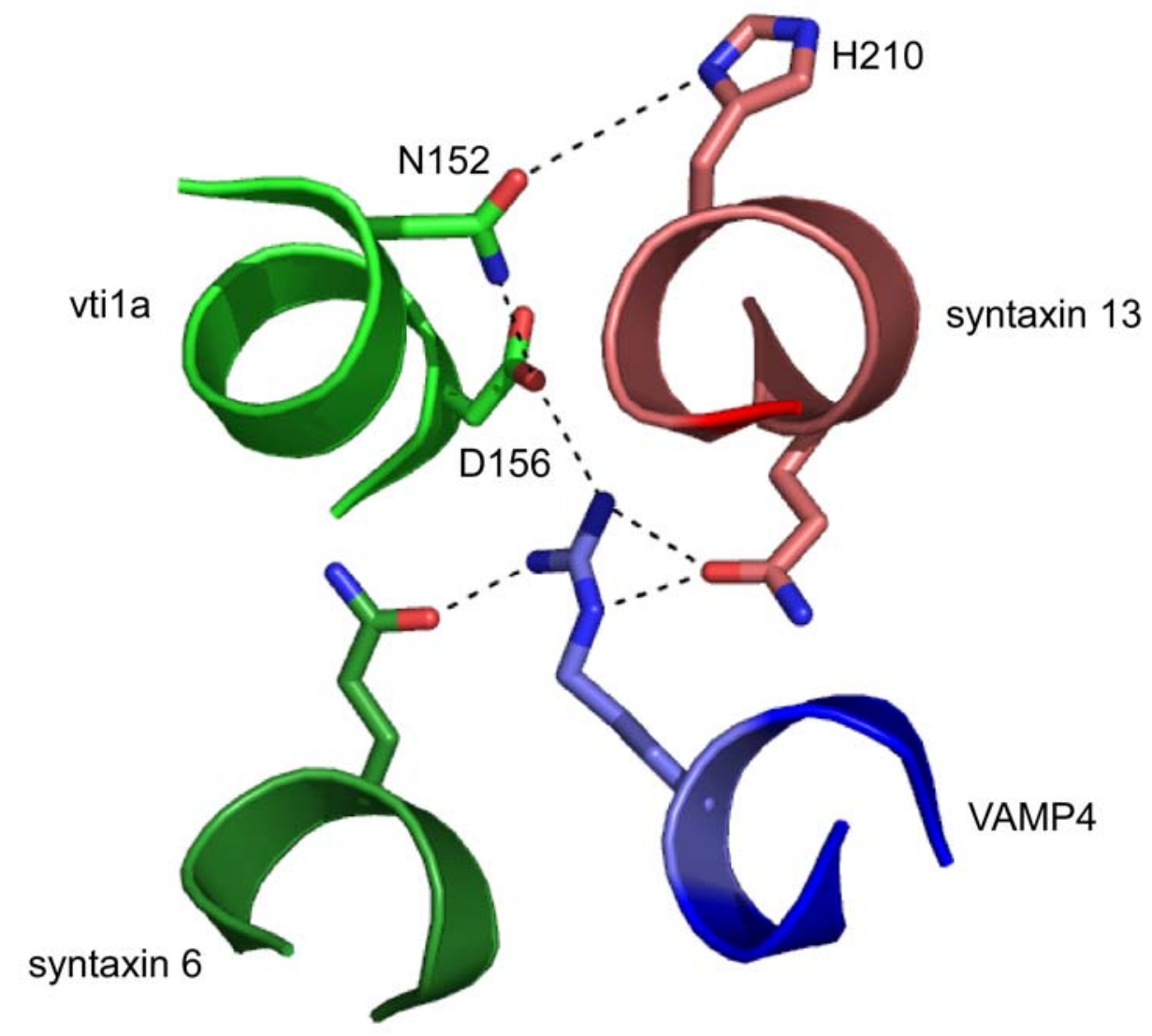

Figure $18 \quad 0$-layer of the Early Endosomal Complex and Additional lonic Interactions with Side Chains. View from carboxy- to aminoterminus into the complex, showing the 0-layer with the four ionic amino acids. An additional intermolecular salt bridge can be observed between vti1 $\mathrm{a}^{\mathrm{N} 152}$ and $\mathrm{s} \times 13^{\mathrm{H} 210}$. The arginine $\left(\mathrm{vti1}^{\mathrm{N} 152}\right)$ also interacts with the 0 -layer amino acid aspartate $\left(v_{t i 1} a^{\mathrm{b} 156}\right)$.

\subsection{5 +6 Layer}

In the +6 layers, SNARE complexes were found to have different modes of layer interactions and stabilisation. The early endosomal and the neuronal complex adapted a situation which is based on hydrophobic interactions, the common interaction in the layers in general (Figure 19). In comparison, the late endosomal syntaxin 8 contains an unusual glutamate in layer +6 which is turned to the outside. This glutamate is stabilised by an arginine in vti1b (Antonin et al., 2002b). Both the glutamate (E200) in syntaxin 8 and the arginine (R192) in vti1b 
are evolutionary conserved residues in the respective isoforms of syntaxin 8 and vti1b. Layer +6 is one of the layers in the complex that exhibit asymmetric complementarity with a bulky phenylalanine and a small alanine and serine.

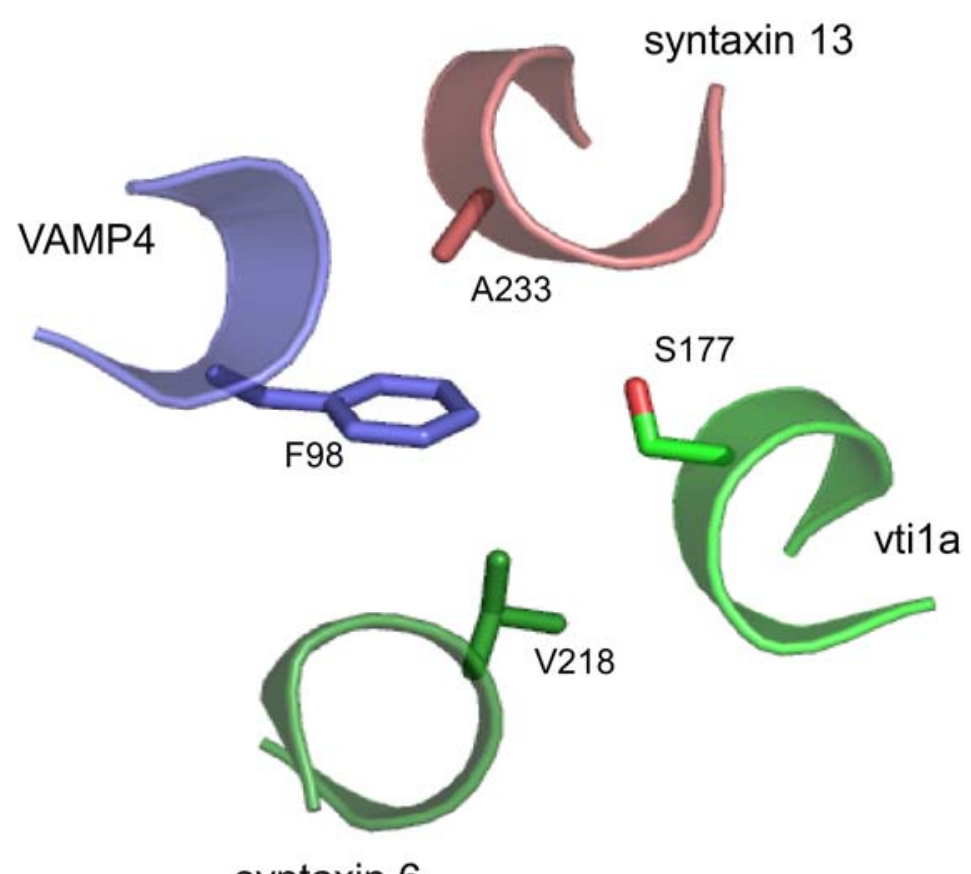

syntaxin 6

Figure 19 Layer +6 of the Early Endosomal SNARE Complex. The layer +6 of the early endosomal complex is stabilised by the hydrophobic interactions of the residues in this layer. The neuronal complex has a similar configuration in layer +6 , whereas the late endosomal complex has an unusual glutamate in this layer that is stabilised by an arginine residue in vti1b. Carboxy- to amino-terminal view. Amino acids of the layer +6 are shown as sticks.

\subsubsection{Interacting Residues are Conserved in Different Positions in the Respective SNAREs}

A stabilising interaction between syntaxin 13 and vti1a was found close to layer -6 involving a glutamate and an arginine (Figure 20a). These residues (vti1 $\mathrm{a}^{\mathrm{R} 131}$ and $\mathrm{s} \times 13^{\mathrm{E} 192}$ ) form a salt bridge. A similar interaction between glutamate and arginine was found in the late endosomal and also in the neuronal complex (Antonin et al., 2002b). However, the residues are conserved at different positions in the $Q_{a}$ - and $Q_{b}$-chains, respectively (Figure 20b). 


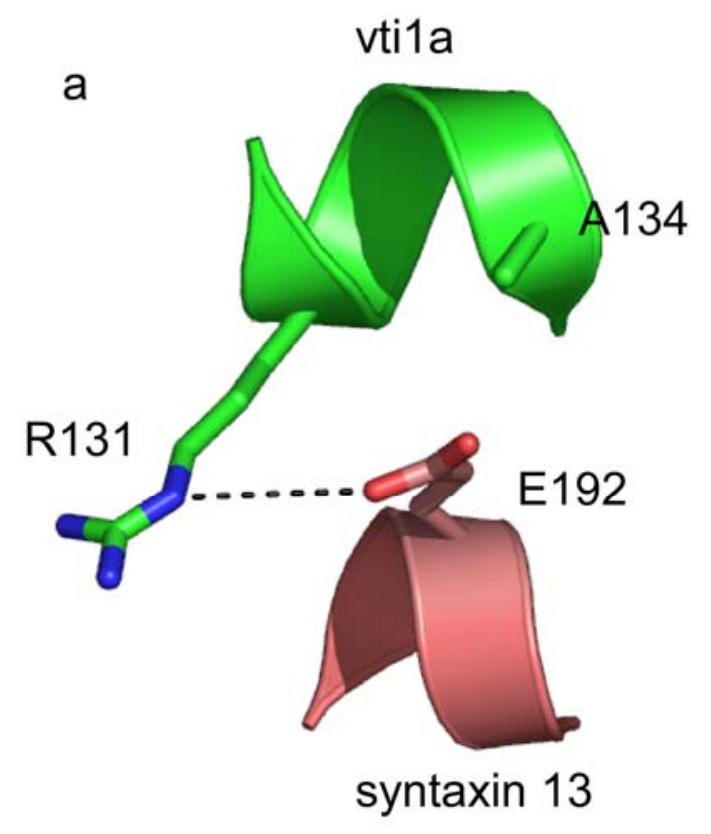

b

$$
\text { vti1a vti1b }
$$

\begin{tabular}{r|ll}
$\begin{array}{r}\text { Homo sapiens } \\
\text { Rattus Norvegicus }\end{array}$ & REEA & SIER \\
Mus musculus & REEA & SIER \\
Drosophila melanogaster & RLTE &
\end{tabular}

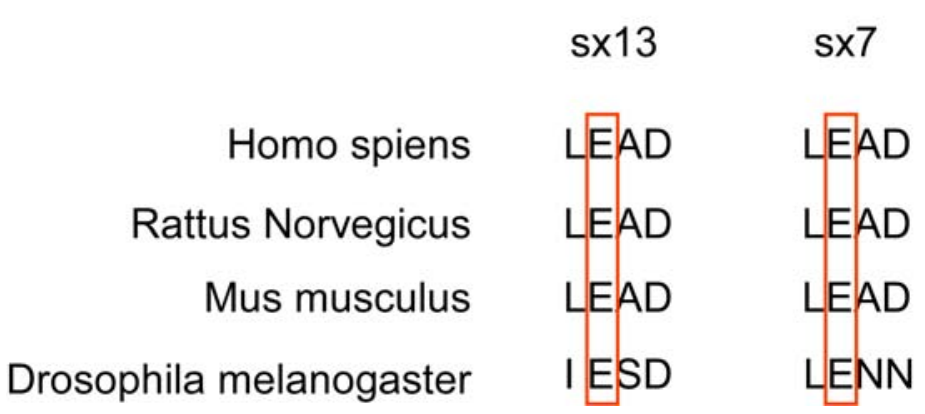

Figure 20 Intermolecular Stabilisation between Sx13 and Vti1a via Alternative Interactions. a) Arginine 131 in vti1a forms a salt bridge with glutamate 192 in syntaxin 13. b) The arginine (R) residue is conserved in vti1a and vti1b (blue box) but they are found at different positions. Both residues, however, interact with the conserved glutamate $(E)$ in the cognate $Q_{a}$-chain (red box). In Drosophila only one vti1 gene is found. The position of the conserved arginine is the same as in vti1a in other species. 
A second example of an alternative binding, that seems to be conserved in the vti1a homologues, is the interaction between the $Q_{b}$ - and the $Q_{c}$-helix, vti1a and sx6, close to layers -4 and -5 (Figure 21). Here, a glutamate (vti1 $a^{\mathrm{E} 143}$ ) exhibits a salt bridge to a serine $\left(s \times 6^{S 179}\right)$. In addition, two neighbouring valine residues form a sandwich with the glutamate $\left(V_{t i 1} a^{E 143}\right)$ in the middle. The valine residues are probably stabilised by hydrophobic interactions. The late endosomal complex only has an aspartate in vti1b and an arginine in syntaxin 8 that interact with each other. The position of the described arginine in syntaxin 8 is found three residues downstream compared to serine in syntaxin 6 (Figure 21b).

a

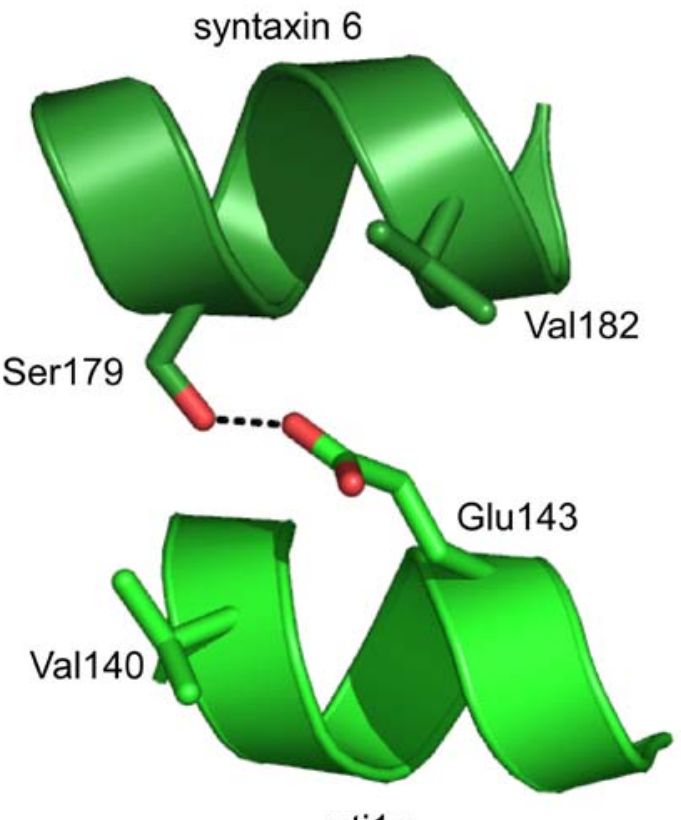

vti1a 
b

\begin{tabular}{rrr} 
Homo sapiens & SIGV & I I SR \\
Rattus Norvegicus & SIGV & I I SR \\
Mus musculus & SIGV & I I SR \\
Drosophila melanogaster & SIGT & TLSR \\
\hline Homo sapiens & VETE & TETD \\
Rattus Norvegicus & VETE & AETD \\
Mus musculus & VETE & TETD \\
Drosophila melanogaster & LETE &
\end{tabular}

Figure 21 Surface Interaction of the $\mathbf{Q}_{b}$ - with the $\mathbf{Q}_{c}$-chain. a) The glutamate 143 in vti1a is sandwiched by two valine residues $\left(\mathrm{s} \times 6^{\mathrm{V} 182}\right.$ and $\mathrm{vti1} \mathrm{a}^{\mathrm{V} 140}$ ) and it forms a salt bridge with a serine in syntaxin 6. b) The glutamate (E) and the serine (S) (blue boxes) in syntaxin 6 and vti1a are conserved residues that interact with each other. The arginine (R) and the aspartate (D) in syntaxin 8 and vti1b of the late endosomal complex are conserved residues that establish a salt bridge (black boxes).

\subsection{Characterisation of SNARE Mediated Liposomal Fusion}

\subsubsection{Fusion of the Proteoliposomes Containing the Early Endosomal SNAREs}

One important approach to investigate the role of SNARE proteins in membrane fusion is the fusion of proteoliposomes (Weber et al., 1998). SNARE proteins incorporated into liposomes are sufficient to induce two different liposome populations to fuse, as long as they are incorporated in such a way, that all four cognate SNAREs of a SNARE complex are inserted in the correct topological combination To test whether the early endosomal SNARE complex is actually able to promote fusion of liposomes, the SNAREs were incorporated in a R + QQ-Q combination and fusion was monitored. The assay that was used to investigate the fusion of liposomes is the so called dequenching assay (Struck et al., 1981). This assay is based on the fact, that two lipid bilayers of two fusing liposomes merge and that lipid mixing occurs. One of the liposome populations, the donor liposomes, contains two lipid-bound dyes, NBD and Rhodamine. The 
term donor is used because this is the population of liposomes that provides the label. The unlabeled ones are the acceptor liposomes. NBD and Rhodamine constitute a FRET pair and Rhodamine quenches the NBD fluorescence as long as they are concentrated in the donor liposomes. When a donor liposome fuses with an acceptor liposome lipid mixing occurs and the two dyes are spatially separated, because the area into which the labels can diffuse increases and NBD is dequenched. The fusion of liposomes thus can be monitored by the increase of NBD signal (Figure 22). The reaction is normalised to total dequenching by adding detergent (Triton X-100), which solubilises the liposomes and separates NBD and Rhodamine maximally in space. 


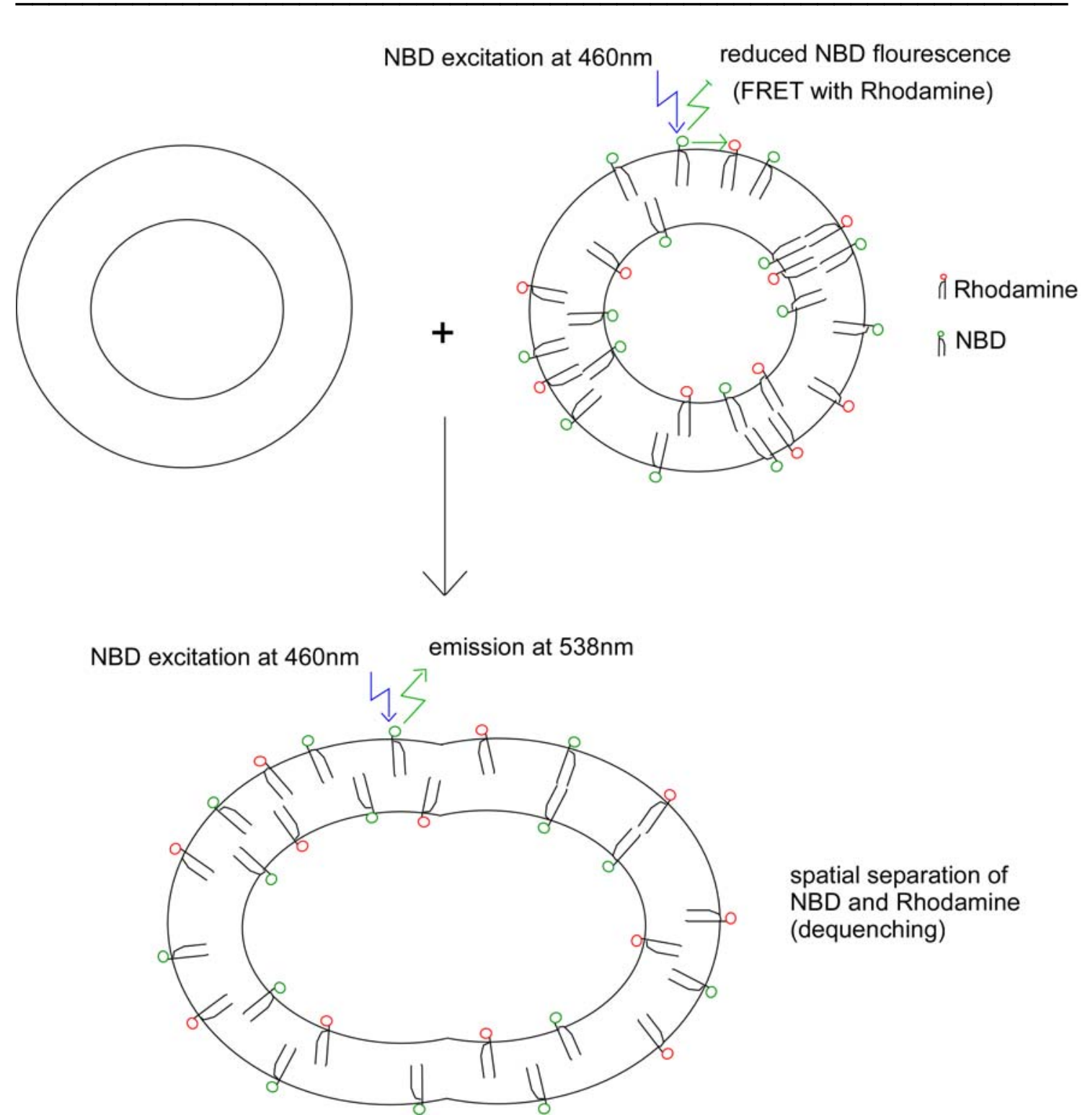

Figure 22 Principle of the Dequenching Assay. Upon fusion, the two dyes NBD and Rhodamine become spacially separated. If inserted into the same liposome, Rhodamine quenches the NBD fluorescence. When the bilayers merge and the dyes are separated, the increase of NBD fluorescence is used as the readout for fusion.

The fusion of VAMP4-liposomes with Q-SNARE liposomes (containing sx6sx13-vti1a) is depicted in Figure 23. The dequenching values usually reached 8$12 \%$ of the total dequenching signal and this is less than was observed in liposomal fusion with the neuronal SNAREs with $40-50 \%$ of total dequenching (Schuette et al., 2004). The kinetics of the fusion reactions were much faster in the early endosomal case than with the neuronal SNAREs. The half maximal value was reached after approx. 3 minutes, whereas, the neuronal SNAREs 
promote fusion with a half time of approximately 20 minutes at comparable protein and lipid concentrations.

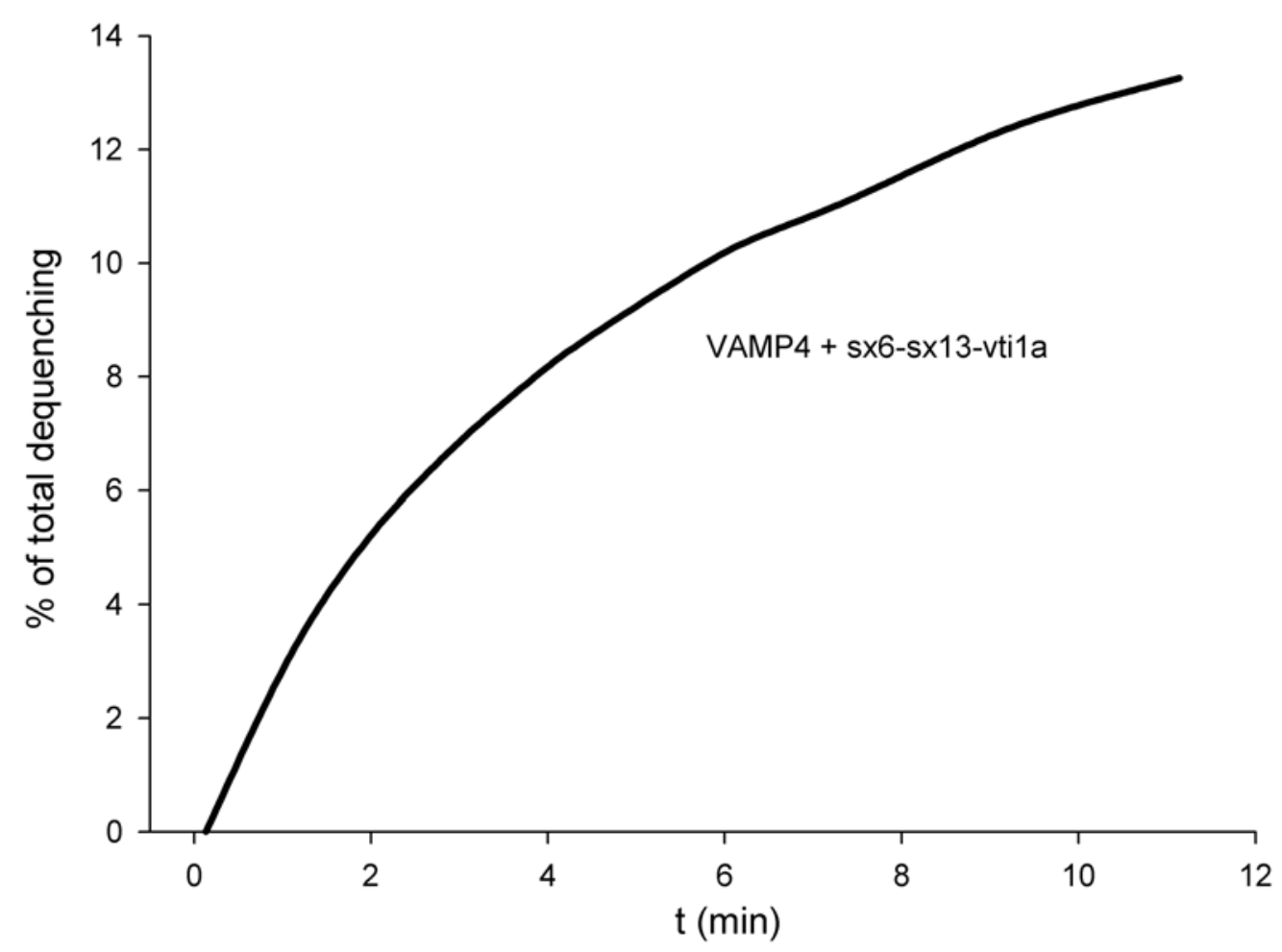

Figure 23 Example for the Fusion Reaction VAMP4 + sx6-sx13-vti1a. The donor liposomes contained VAMP4 and the acceptor liposomes the early endosomal Q-SNAREs. The donor liposomes were used with $1.5 \mu \mathrm{M}$ and the acceptor liposomes with $3 \mu \mathrm{M}$ final concentration for each protein.

\subsubsection{Inhibition of Liposomal Fusion with Soluble R-SNARE Fragments}

In order to test whether the observed fusion is SNARE dependent (to exclude unspecific dequenching effects), the cytoplasmic region of VAMP4 was added in increasing concentrations to the reaction as a competitive inhibitor, while both liposome populations were adjusted to $3 \mu \mathrm{M}$ protein concentration (final protein concentration of each protein). The inhibition of liposome fusion was concentration dependent and fusion was almost fully inhibited by adding high amounts of VAMP4 (Figure 24 a). Adding the three Q-SNAREs (sx6, sx13, vti1a) to the R-SNARE liposome as competitors also inhibited fusion but inhibition was not as efficient, probably because three proteins need to 
assemble simultaneously on the R-SNARE. Thus, it was shown that the fusion reaction VAMP4 + sx6-sx13-vti1a was SNARE dependent. Fusion can be inhibited using soluble SNAREs in a dose dependent manner.
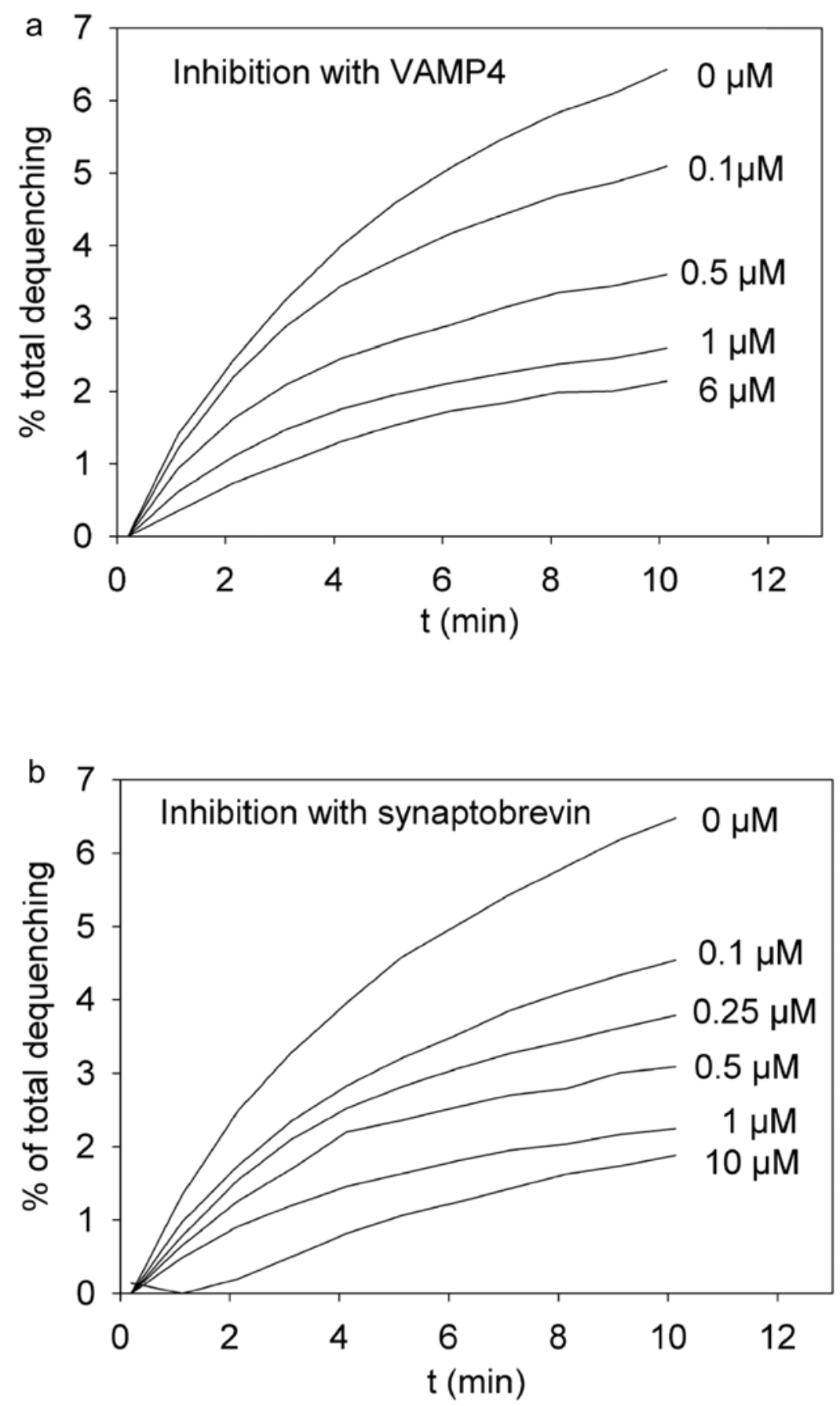


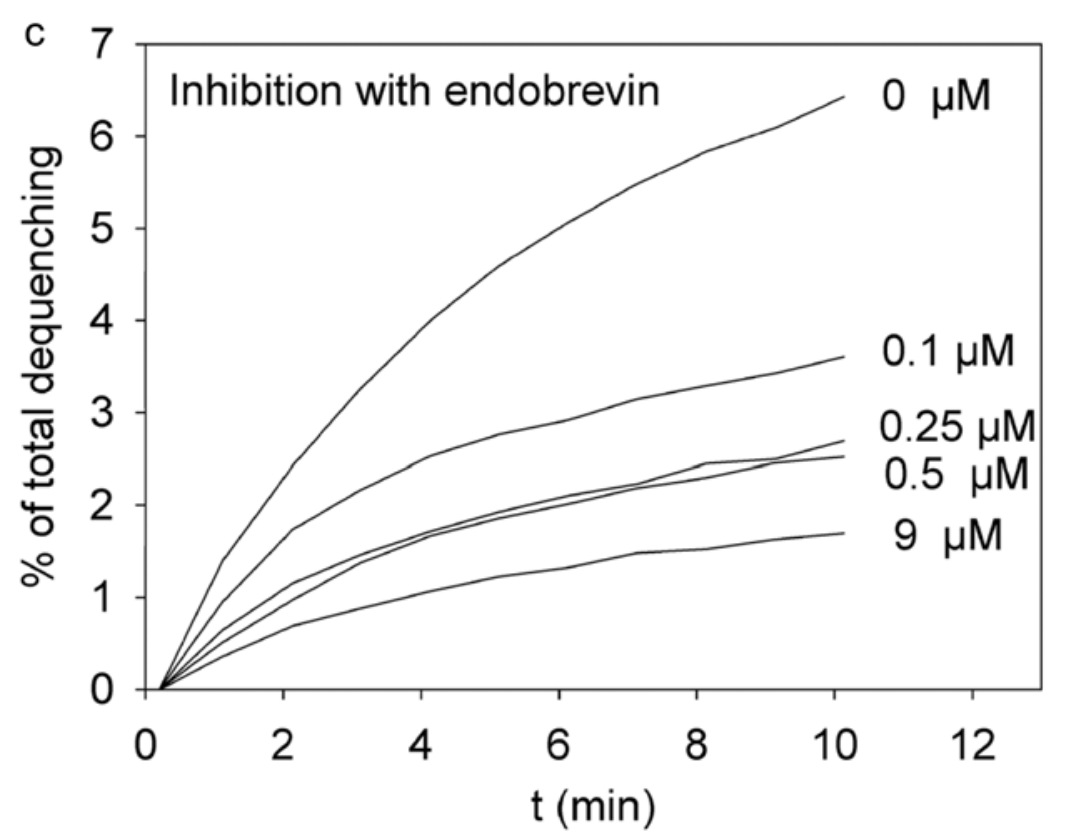

Figure 24 Dose-dependent Inhibition of Liposome Fusion by Increasing Amounts of Different Soluble R-SNAREs. The rate of VAMP4liposomes fusing with Sx6-Sx13-Vti1a-liposomes decreases when increasing amounts of soluble VAMP4 (a), synaptobrevin (b), or endobrevin (c), are added to the reaction mix $(0-10 \mu \mathrm{M}$, final concentration of soluble R-SNAREs as indicated). Measurements were performed at $37^{\circ} \mathrm{C}$ with a protein concentration of $3 \mu \mathrm{M}$ in the liposomes. The soluble R-SNAREs were added to the Q-SNARE liposomes simultaneously with the R-SNARE liposomes after temperature equilibration to $37^{\circ} \mathrm{C}$.

As described in 3.1.3 the ability of different R-SNAREs to inhibit fusion of early endosomal from PC12 cells varies. Only VAMP4 specifically inhibited the fusion of early endosomes. All other R-SNAREs that were investigated did not have an effect on fusion (except endobrevin to a minor extent). To check whether the specificity of the R-SNAREs differs also in vitro the three R-SNAREs VAMP4, endobrevin and synaptobrevin were used to inhibit the liposomal fusion reaction VAMP4 + sx6-sx13-vti1a. Increasing amounts of endobrevin and synaptobrevin were added to the fusion reaction (Figure $24 \mathrm{~b}$ and $\mathrm{c}$ ). Adding the two RSNAREs inhibited fusion with similar efficiencies as with soluble VAMP4 fragments. To be able to compare the efficiencies of the different R-SNAREs the half maximal inhibitory concentrations $\left(\mathrm{IC}_{50}\right)$ for each protein were calculated for each experiment. $I_{50}$ values thus serve as comparable parameters for the potency of the SNAREs. Each set of experiments was analysed by plotting the percent inhibition as a function of the natural logarithm 
(In) of the concentration of soluble inhibitory SNARE fragment. The inhibition should follow a dose-response curve and should have a sigmoidal shape. An example is depicted in Figure 25. It shows the analysis of the reaction in Figure $24 a$.

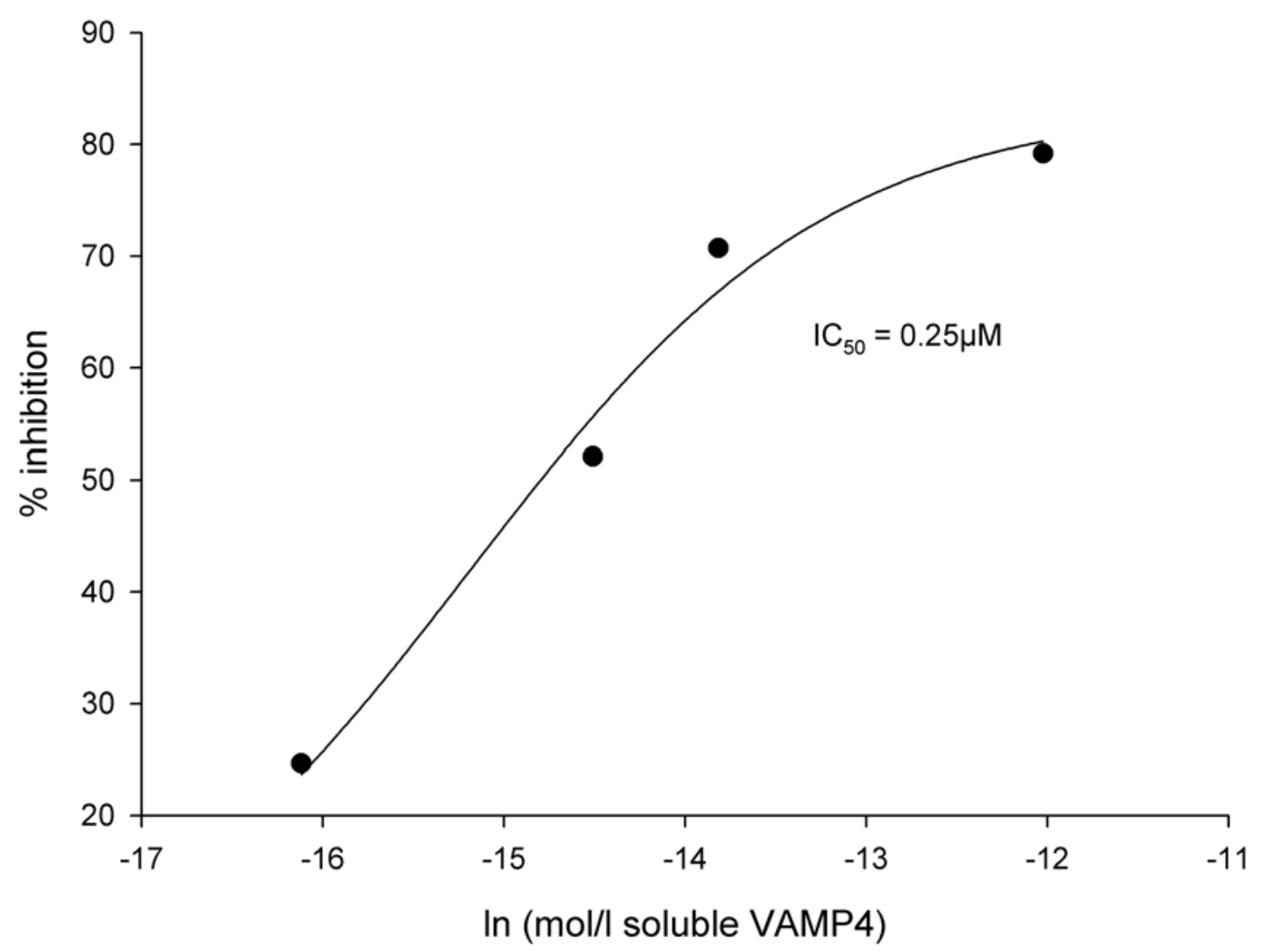

Figure 25 Inhibition Experiments can be Evaluated by Plotting Inhibition vs. Concentration of Soluble Protein and Fitting the Trace with a Sigmoidal Function. The normalised dequenching signals were converted to $\%$ inhibition and then plotted against the natural logarithm (In) of the concentration of soluble SNARE. This can be fitted with the function $y=a / 1+\exp \left(-\left(x-x_{0} / b\right)\right)$. Liposomes were adjusted to $3 \mu \mathrm{M}$ protein final concentration.

The three R-SNAREs inhibit the fusion reaction in a dose dependent manner, and in the same concentration range in all three cases. These experiments were performed several times. VAMP4 has an average $\mathrm{IC}_{50}$ of $0.62 \mu \mathrm{M}$, endobrevin has an average $\mathrm{IC}_{50}$ of $0.45 \mu \mathrm{M}$ and synaptobrevin an average $\mathrm{IC}_{50}$ of $0.16 \mu \mathrm{M}$ while the liposomes were used at a final concentration of $3 \mu \mathrm{M}$ per protein (Table 4). Proteolytic digest demonstrated that virtually all SNAREs face the outside of the liposome (section 2.13, Figure 3). In addition, experiments 
were performed including a $1 \mathrm{~h}$ preincubation time of the R-SNAREs with the QSNARE liposomes. In these experiments endobrevin and synaptobrevin inhibited with $\mathrm{IC}_{50}$-values of $0.2 \mu \mathrm{M}$ (endobrevin) and $0.18 \mu \mathrm{M}$ (synaptobrevin), the same order of magnitude as in the experiments without preincubation. However, VAMP4 had a higher potency in these experiments and the $\mathrm{IC}_{50}$ was $0.05 \mu \mathrm{M}$ (data not shown). This effect is difficult to explain since the soluble VAMP4 is present at a 60 -fold lower concentration than the protein on the liposomes, thus secondary effects of VAMP4 unrelated to SNARE binding, cannot be excluded. This experiment demonstrated that the Q-SNAREs in the liposome can interact with soluble R-SNAREs different from cognate VAMP4, exhibiting promiscuity.

\begin{tabular}{|c|c|c|c|}
\hline $\begin{array}{c}\text { Fragment used } \\
\text { for inhibition }\end{array}$ & $\begin{array}{c}\text { VAMP4 } \\
\mathbf{1 - 1 1 7}\end{array}$ & $\begin{array}{c}\text { endobrevin } \\
\mathbf{1 - 7 4}\end{array}$ & $\begin{array}{c}\text { synaptobrevin } \\
\mathbf{1 - 9 6}\end{array}$ \\
\hline \multirow{2}{*}{$\begin{array}{c}\text { Calculated } \\
\text { IC }_{50}(\mu \mathrm{M})\end{array}$} & 0.25 & 0.75 & 0.21 \\
& 1 & 0.32 & 0.15 \\
\hline $\begin{array}{c}\text { Average } \mathrm{IC}_{50} \\
(\mu \mathrm{M})\end{array}$ & $\mathbf{0 . 6 2}$ & 0.69 & 0.13 \\
\cline { 2 - 4 } & & 0.06 & 0.16 \\
\hline
\end{tabular}

Table 4 Calculated $I_{50}$ Values for the Three R-SNAREs VAMP4, Endobrevin and Synaptobrevin. All inhibition experiments were analysed and the calculated $\mathrm{IC}_{50}$ values and the averages of all experiments are listed.

\subsubsection{Different R-SNARE Liposomes are Able to Fuse with the Q- SNARE Liposomes}

As the three soluble R-SNAREs, VAMP4, endobrevin and synaptobrevin were equally potent in inhibiting liposomal fusion, it was tested whether these SNAREs are also able to promote fusion when reconstituted into liposomes. Studies by another group suggested that SNAREs encode compartmental specificity and that SNAREs coming from one compartment do not promote fusion of liposomes with R-SNAREs from another compartment (McNew et al., 2000). The R-SNAREs were incorporated into liposomes and fusion 
experiments were performed using a protein concentration of $3 \mu \mathrm{M}$ for each liposome population.

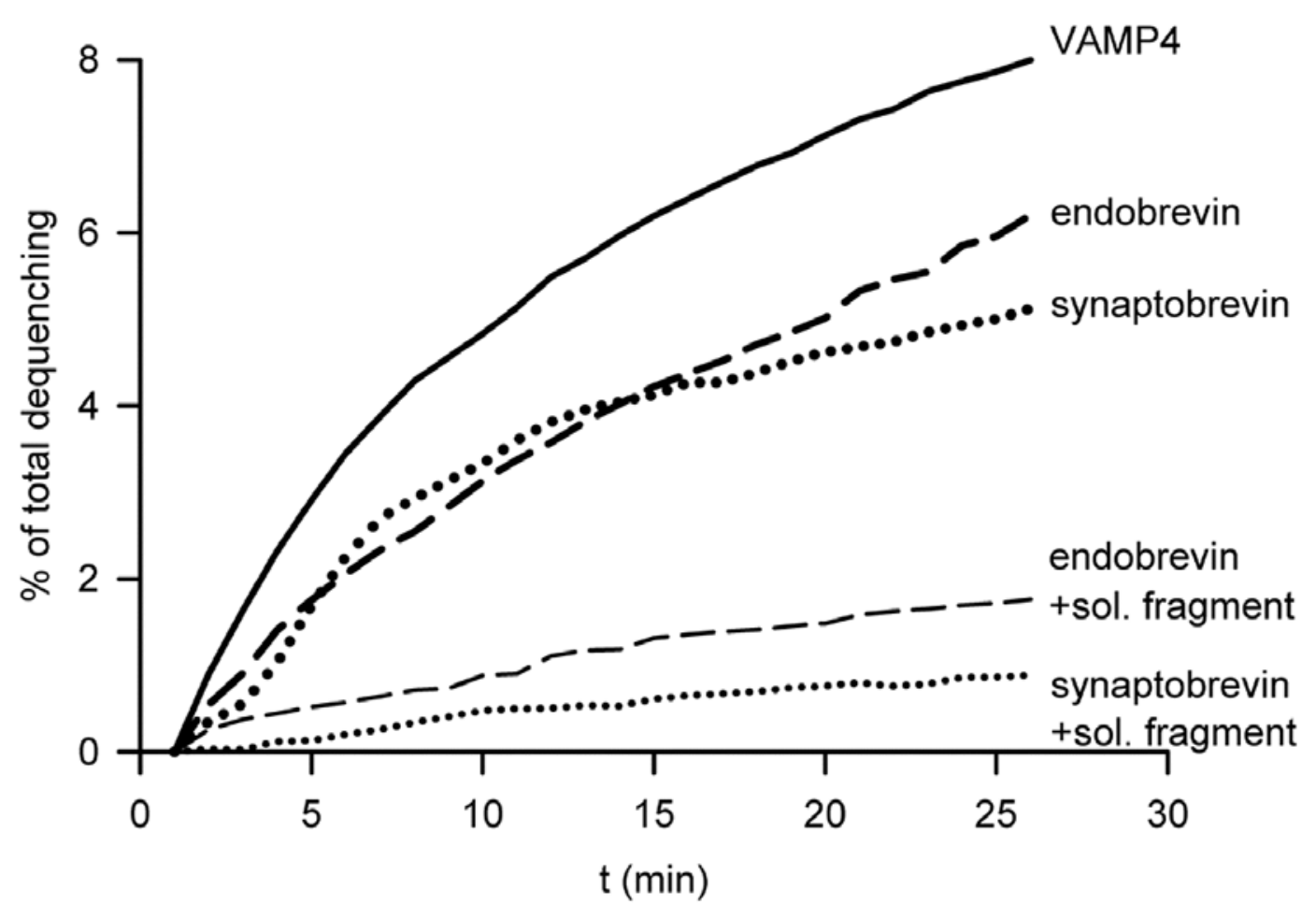

Figure 26 Different R-SNARE Liposomes Fuse with the Q-SNARE Liposomes Containing Sx6, Sx13 and Vti1a. Proteoliposomes containing the Q-SNAREs syntaxin 13 , syntaxin 6 , and vti1a fuse with R-SNARE liposomes containing VAMP4, endobrevin, or synaptobrevin. As negative control, Q-SNARE liposomes were preincubated for $1 \mathrm{~h}$ at room temperature with purified endobrevin or synaptobrevin lacking the transmembrane domain (sol. fragment, final concentration $30 \mu \mathrm{M}$ ) before starting the fusion reaction.

The R-SNARE liposomes containing endobrevin and synaptobrevin are able to fuse with Q-SNARE liposomes that contain sx6, sx13 and vti1a (Figure 26). The results of all experiments with different R-SNARE liposomes fusing with the QSNARE liposomes are summarised in Figure 27. This experiment confirmed the inhibition experiments with soluble R-SNAREs. Non-cognate SNAREs are able to interact and promote fusion at least in the combination of sx6-sx13-vti1a liposomes with endobrevin or synaptobrevin containing liposomes. 


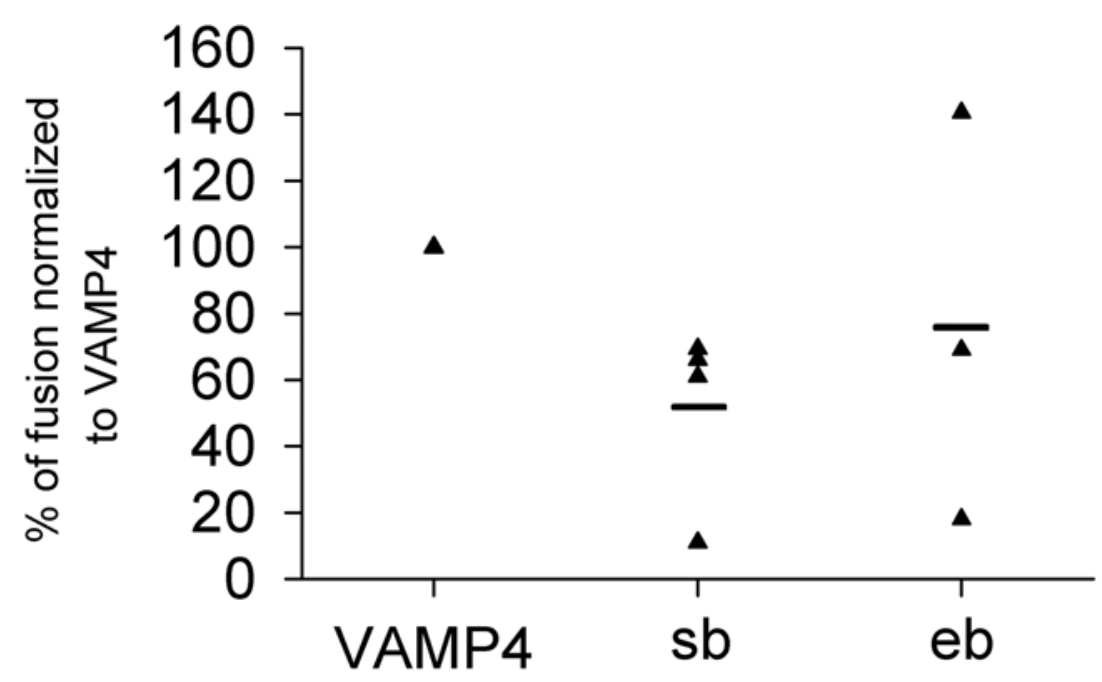

Figure 27 Fusion of Different R-SNARE Liposomes with Q-SNARE Liposomes Containing Sx6, Sx13 and Vti1a. The fluorescence signals of fusion reactions at $\mathrm{t}=10 \mathrm{~min}$ were normalised to the signals measured with VAMP4. Results were derived from experiments in which either the R-liposomes or the Q-liposomes were NBD/Rho labelled. The values of different experiments that were obtained with the same preparation of liposomes were averaged and plotted as one triangle. Total number of fusion reactions were VAMP4 $=9$, synaptobrevin $=9$ and endobrevin $=4$. The mean values of all reactions are indicated by horisontal bars. Fusion with synaptobrevin liposomes resulted in an average of $51 \%$ and fusion with endobrevin liposomes of $76 \%$. The two exceptionally low values for synaptobrevin $(11 \%)$ and endobrevin $(18 \%)$ were obtained the same day from one liposome preparation and may not be typical.

To summarise all data, specific interaction of the SNAREs was observed in the fusion assay using PC12 early endosomes. However, in the in vitro situation using proteoliposomes this specificity was no longer observed. Soluble SNARE fragments of different R-SNAREs act as competitive inhibitors and the fulllength R-SNAREs are able to mediate fusion with similar efficiencies.

\subsubsection{Topology-Dependent Fusion Investigated with the Dequenching Assay}

The fusion of liposomes with SNARE proteins inserted in different topological combinations was investigated by the Rothman group. It was claimed that 
fusion only occurs if the SNAREs are distributed in a $1 \mathrm{v}$-SNARE (Bet1) to $3 \mathrm{t}$ SNARE (Sed5-Bos-Sec22) combination i.e. one liposome population contains the so-called V-SNARE and the other population contains the three t-SNAREs of the respective SNARE complex. Any other combination, did not result in fusion (Parlati et al., 2000). Additionally, any combination missing one of the SNARE proteins failed to fuse. The experiments included the yeast ER to Golgi SNAREs Bet, Bos, Sec22 and Sed5 (Parlati et al., 2000). To test whether the same applies to the early endosomal SNAREs similar experiments were performed. The SNARE proteins VAMP4, sx6, sx13 and vti1a were distributed over 14 different liposome populations in all possible non-redundant 1:3 or 2:2 combinations (Figure 28).

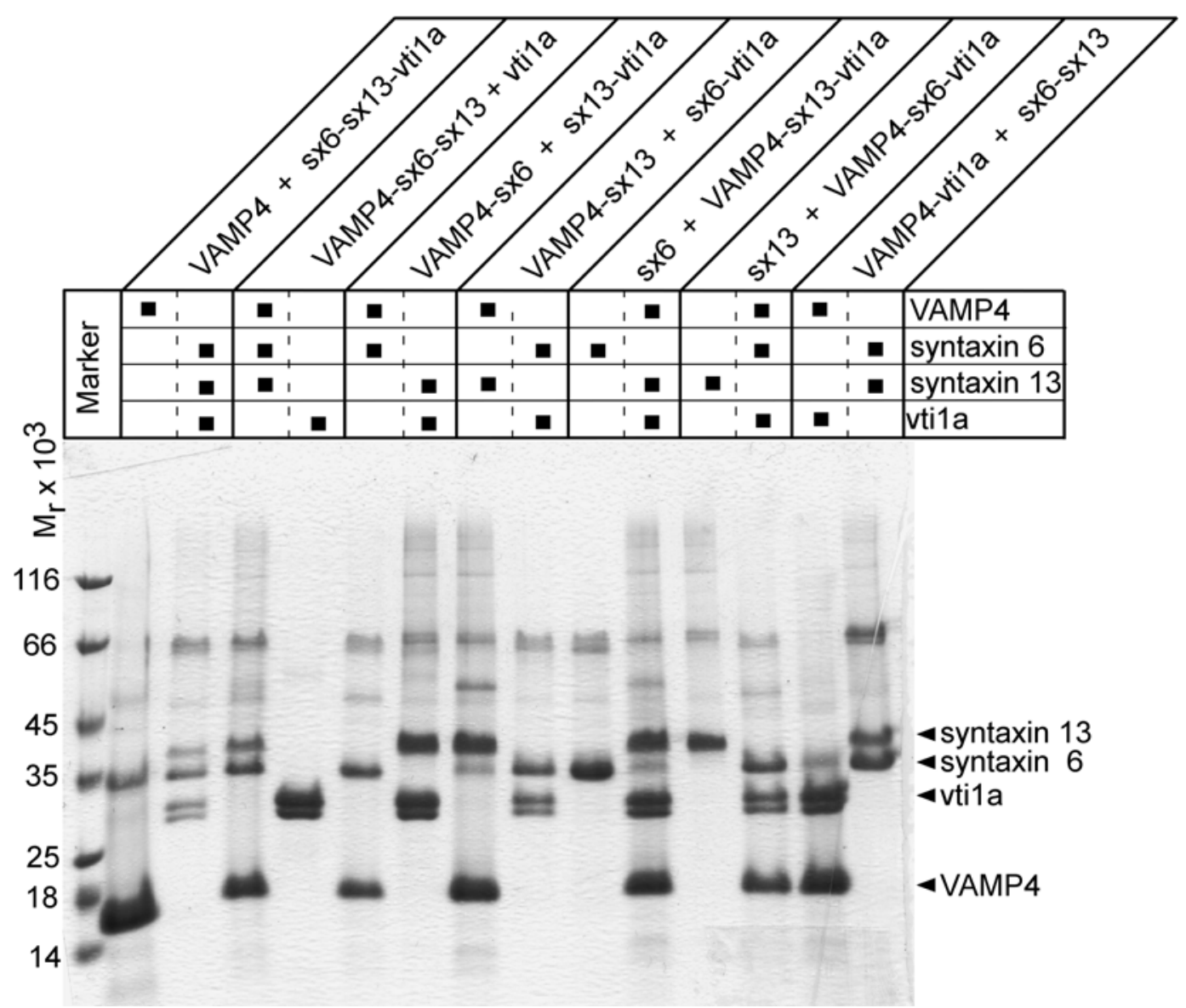

Figure 28 SDS-PAGE of all 14 Different Liposome Populations that Contained the 1:3 or 2:2 Topological Combinations. The gel represents 7 blocks of pairs of liposomes complementing each other. These were combined in fusion experiments to determine whether the respective topological combinations result in fusion. $10 \mu \mathrm{l}$ of each liposomal preparation were loaded on a $10 \%$ Schaegger SDS gel. The gel was stained with Commassie Blue. 
The combinations were analysed by SDS-PAGE by loading the two fusion partners next to each other (e.g. VAMP4 and sx6-sx13-vti1a). Each protein was efficiently incorporated into the liposomes but some populations (e.g. sx6-sx13vti1a contained less protein than others. Each pair of liposomes was used for fusion experiments. Rhodamine/NBD label was incorporated in one population (donor liposomes), the liposomes that contained one SNARE or two SNAREs including VAMP4 (Figure 29). For better signal to noise ratio the protein concentrations of donor and acceptor liposomes were adjusted to $1.5 \mu \mathrm{M}$ (donor) and $3 \mu \mathrm{M}$ (acceptor).

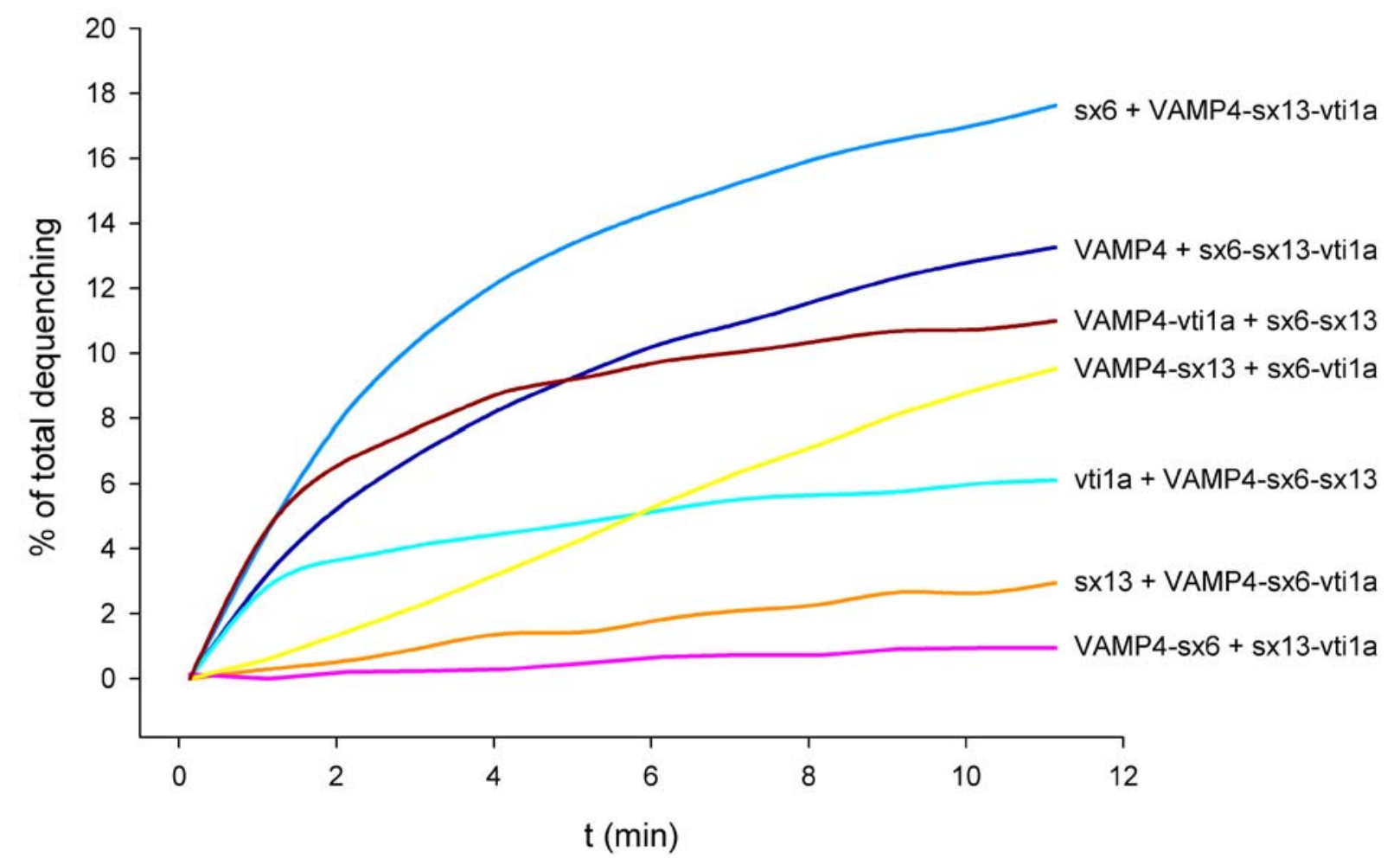

Figure 29 Liposomes Containing all Possible Non-Redundant Topological 1:3 or 2:2 Combinations of SNAREs. Depicted are the kinetics of the 7 fusion reactions in which the liposomes from Figure 28 were used. Only the reaction with liposomes containing sx13 $\left(Q_{a}\right)$ (orange) and VAMP4-sx6 (pink) do not show fusion with their cognate partner. The reaction VAMP4-sx13 + sx6-vti1a (yellow) in this experiment shows an unusual kinetic, the example is not representative for this topological combination.

The different fusion reactions exhibited different kinetics and maximal dequenching signals. The dequenching values after $10 \mathrm{~min}$ varied between $6 \%$ and $16 \%$ (Figure 29). This is less than the values that were observed for the neuronal or late endosomal SNAREs (Schuette et al., 2004). To compare the 
reactions the dequenching values at $\mathrm{t}=10 \mathrm{~min}$ were taken and depicted in a bar diagram (Figure 30). The results of 4 different reactions from two liposome preparations were averaged (black bars). The capability for fusion was also checked after swapping the labels to the other population. A new set of experiments was performed, this time dequenching 'in the other direction' (grey bars).

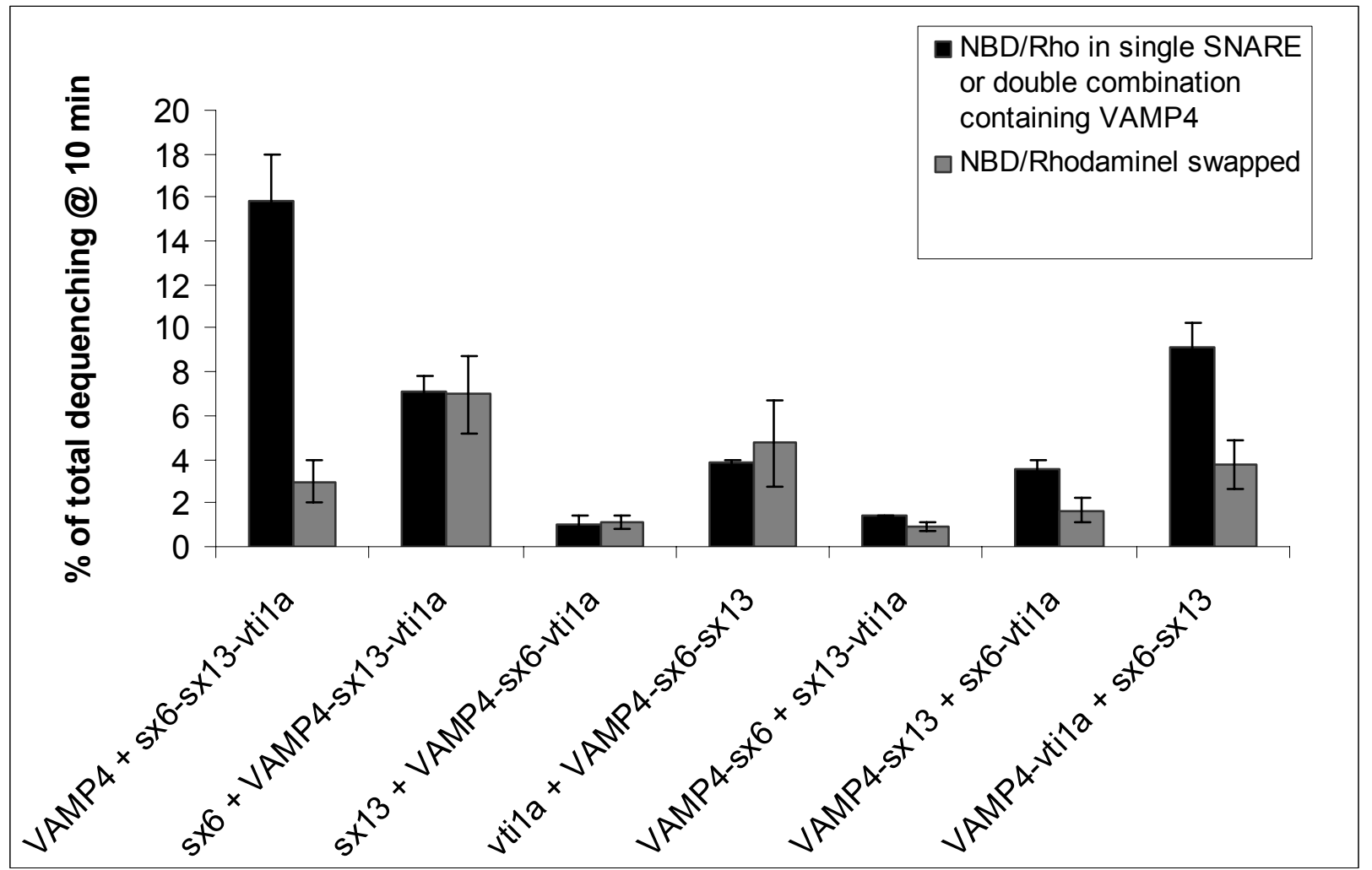

Figure 30 Evaluation of the Topological Combinations in Liposomal Fusion using the Dequenching Assay. The bar diagram shows the results of 8 different sets of experiments. The SNAREs that are stated first (before the + ) were incorporated in the NBD/Rhodamine liposomes (four experiments from two independent liposomal preparations). For each liposomal preparation the experiment was repeated the next day (black bars). Then the NBD/Rhodamine label was swapped and the SNAREs that are stated after the + were incorporated into the NBD/Rhodamine liposomes. Again, a total of 4 sets from 2 liposome preparations were performed (grey bars). Error bar= SEM; $n=4$. 
The values for the different topologies were (mean +/- SEM) (black bar values first, followed by grey bar values):

\begin{tabular}{|c|c|c|}
\hline Topological distribution & $\underline{\text { black bars }}$ & grey bars \\
\hline VAMP4 + sx6-sx13-vti1a & $15.8 \%+/-2.1$ & $2.9 \%+/-0.9$ \\
\hline sx6 + VAMP4-sx13-vti1a & $7.1 \%+/-0.7$ & $6.9 \%+/-1.7$ \\
\hline sx13+ & $1.0 \%+/-0.38$ & $3 \%+/-0.29$ \\
\hline vti1a + VAMP4-sx6-sx13 & $3.8 \%+/-0.12$ & $4.7 \%+/-1.9$ \\
\hline VAMP4-sx6 + sx13-vti1a & $1.39 \%+/-0.06$ & $0.9 \%+/-0.19$ \\
\hline VAMP4-sx13 + sx6-vti1a & $3.58 \%+/-0.34$ & $1.64 \%+/-0.57$ \\
\hline VAMP4-vti1a + sx6-sx13 & $9.1 \%+/-1.1$ & $3.77 \%+/-1.1$ \\
\hline
\end{tabular}

Five combinations show dequenching signals. However, the signals vary among the different combinations. Some topologies may fuse more efficiently than others, depending on the combination of proteins. The reaction sx13 + VAMP4sx6-vti1a and VAMP4-sx6 + vti1a do not show dequenching above background. The exchange of label in one combination (compare black with grey bars) gives divergent results in some cases. Certain combinations of SNAREs tend to precipitate or aggregate the liposomes. As a result, the protein concentration among the different liposome population differs. This may lead to reduced fusion activity.

Although the experiments were performed with fixed protein concentrations $(1.5 \mu \mathrm{M}$ for donor and $3 \mu \mathrm{M}$ for acceptor liposomes) the low protein concentration per individual liposome of some combinations may have resulted in lower fusion efficiencies. This was probably the case for the combination VAMP4 + sx6sx13-vti1a and the combination VAMP4-vti1a + sx6-sx13. Although the buffer for the generation of liposomes contained $1000 \mathrm{mM}$ salt (HB1000) precipitation could not be fully avoided. The donor liposomes needed to be used in lower amounts than the acceptor liposome to maintain better signal to noise ratio. Under these conditions the overall fusion efficiency might have suffered because the protein concentration in the donor liposomes was reduced to a certain level or parts of the liposome populations were incapable of fusion because of clustering. This may have been the case for the two combinations in which swapping of the label did not result in the same fusion efficiencies. 
Despite the fact that some combinations varied in their fusion results, the data indicate, that five out of seven combinations enhance fusion, whereas two of them did not exhibit fusion i.e. sx13 + VAMP4-sx6-vti1a and VAMP4-sx6 + sx13-vti1a.

\subsubsection{Inhibition of Liposome Fusion}

Fusion was not always confirmed reliably in the liposomal fusion experiments after swapping the labels. Therefore, to exclude false positive fusion results, the soluble (cytosolic) SNARE fragments were used in a competitive inhibition experiment. In theory, these soluble SNARE fragments should form cis complexes with SNAREs residing in the liposome, thus competing with the endogenous SNAREs. The SNAREs residing in the liposome should then no longer be able to form trans-complexes with other liposomes, therefore fusion is blocked. Examples of two such reactions is shown in Figure 31. As shown in chapter 3.3.2 adding soluble R-SNAREs to the Q-SNARE liposomes resulted in potent inhibition of fusion in a $R+Q Q Q$ reaction. 


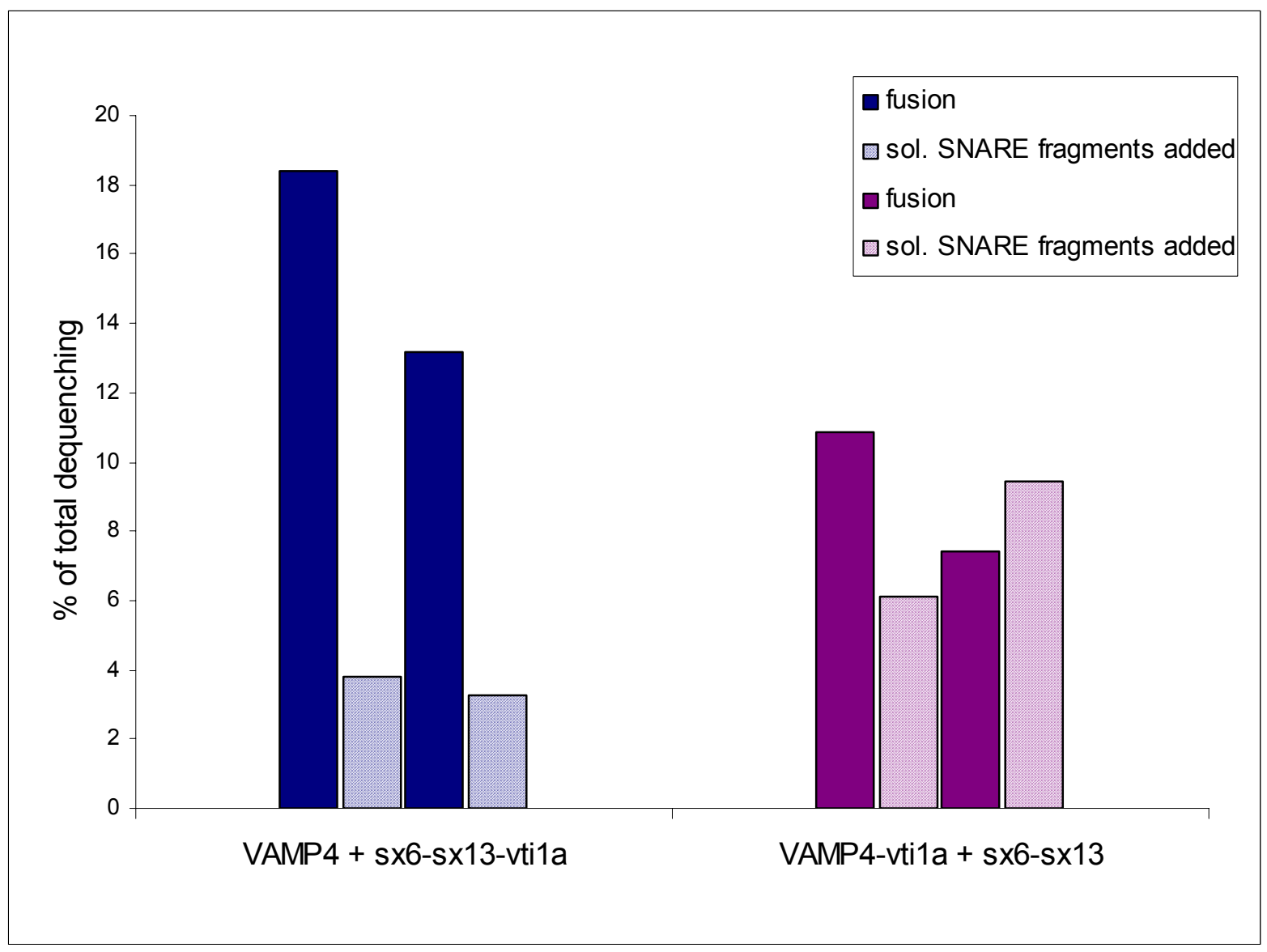

Figure 31 Fusion Cannot be Inhibited in All Cases by Adding Soluble SNARE Fragments. To investigate if the observed fusion is SNARE dependent, soluble SNARE fragments were added to the liposomes $1 \mathrm{~h}$ before the fusion experiment started. The complementary SNAREs were added to one liposomal population for the formation of cis complexes on these liposomes (e.g. soluble sx6 and sx13 were added in a final concentration of 15$30 \mu \mathrm{M}$ to liposomes that contained VAMP4 and vti1a and vice versa). The graph shows two example reactions, each experiment was done in duplicate with the same liposomes. Solid bars, fusion reaction; striped bars, soluble SNARE fragments added.

As depicted in Figure 31 the reaction of VAMP4 (R) liposomes with sx6-sx13vti1a (Q-Q-Q) liposomes was completely inhibited by adding soluble VAMP4 to the Q-SNARE liposomes. However, adding soluble sx6 and sx13 to the VAMP4-vti1a-liposomes did not result in an inhibitory effect. In a different experiments it was observed that mixing both the liposome populations with the respective complementary soluble SNAREs simultaneously did not have a strong effect, either. In some reactions, an inhibition of approximately $70 \%$ was achieved, however, a full block of fusion was never observed. Obviously, the fusion reactions of the type $Q+R Q Q$ or $R Q+Q Q$ behave differently from the 
'standard' $R+Q Q Q$ reaction. In $C D$ spectroscopy slow assembly rates for the core complexes were observed (3.1.6). Slow assembly kinetics might be the reason for inefficient cis-complex formation on the liposomes within the $1 \mathrm{~h}$ preincubation performed.

A different approach was taken to verify SNARE dependent fusion. Labelled liposomes containing proteins were mixed with empty, protein-free, liposomes. Empty liposomes should not fuse with the donor liposomes because the fusion mediators (SNAREs) are absent. Nevertheless, unspecific fusion was observed in some cases. This was probably due to smaller sizes of these empty, proteinfree liposomes and the resulting high surface tension. To circumvent this problem liposomes containing SNAREs were digested with proteases. Liposomes were generated as usual and then digested with trypsin. After this treatment the liposomes are thought to keep their normal diameter, because the transmembrane domains remain integrated in the lipid bilayer while any cytosolic domain the surface of the liposomes would be degraded by the protease. But even under these experimental conditions some residual fusion occurred in some cases. However, the resulting dequenching signal did not follow a typical exponential reaction curve but rather increased in a linear fashion. When comparing the results of experiments in which the tryptic digest was alternated between the liposome populations it became apparent that digesting liposomes containing VAMP4 seemed to have remaining fusion activity. The digestion of VAMP4 might have resulted in the generation of a fusogenic peptide that promotes fusion to some level. The 'one-sided' digest, the digestion of only the liposomes that did not contain VAMP4 (and leaving the other population undigested), was successful in blocking fusion (Figure 32). 


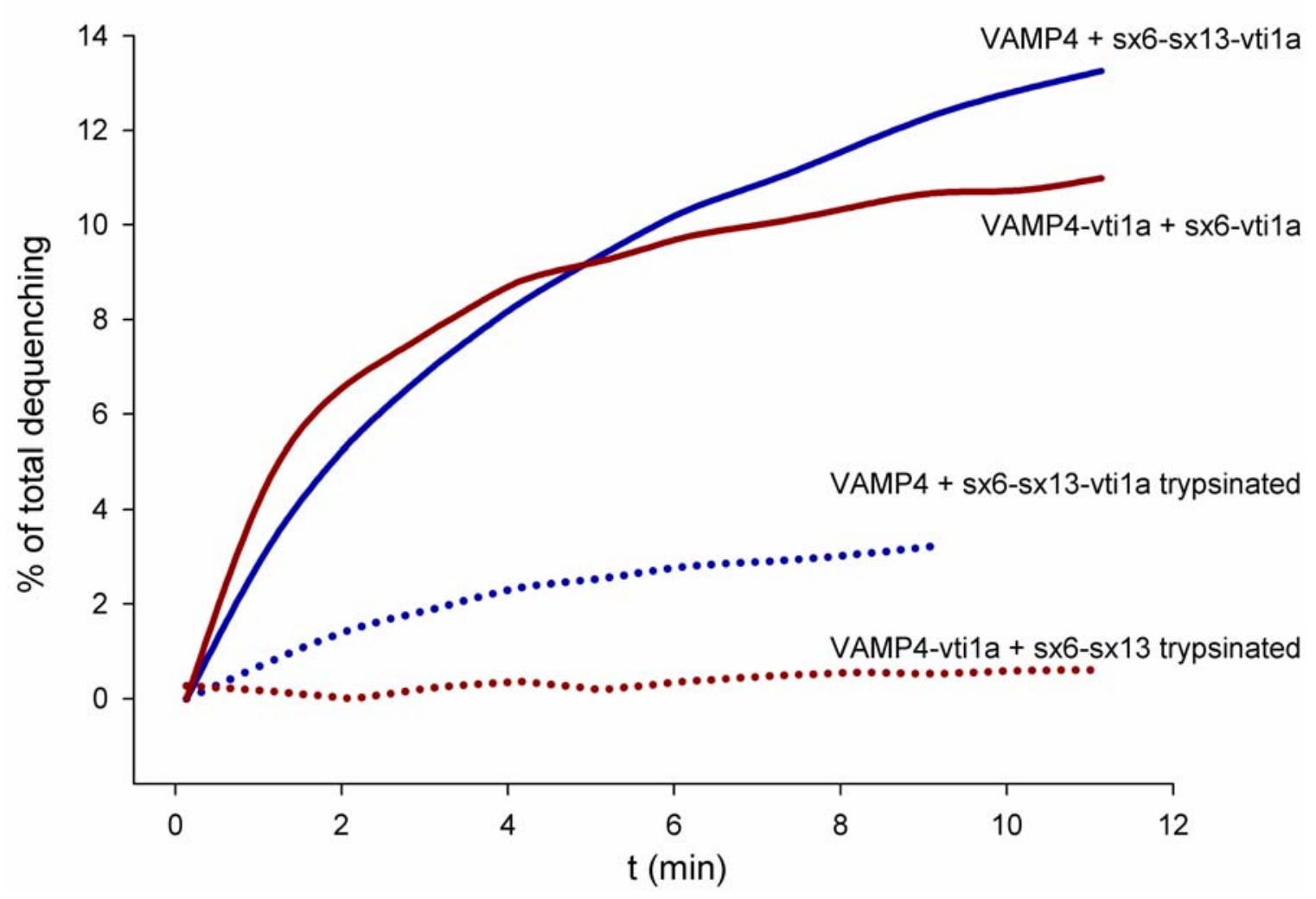

Figure 32 'One-sided' Trypsin Digest of Liposomes Inhibits Fusion Potently. Fusion reactions can be fully inhibited by digesting one of the liposome populations (the one that does not contain VAMP4 in this case). $5 \mu$ l of donor liposomes and $35 \mu \mathrm{l}$ of acceptor liposomes were mixed with $10 \mu$ l buffer after equilibration to $37^{\circ} \mathrm{C}$. For the trypsin digest the respective liposomes were incubated with $5 \mu \mathrm{l}$ of a $1 \mathrm{mg} / \mathrm{ml}$ trypsin solution $1 \mathrm{~h}$ at $37^{\circ} \mathrm{C}$ prior to the experiment.

In further experiments, the signal to noise ratio was reduced by using fixed volumes of liposomes in a donor: acceptor ratio of 1:7. $5 \mu \mathrm{l}$ of donor liposomes were combined with $35 \mu \mathrm{l}$ acceptor liposomes in a total volume of $50 \mu \mathrm{l}$. Fusion was blocked by digestion of the 'non-VAMP4 liposomes'. Examples of fusion reactions are depicted in Figure 33. The signals of the five positive combinations improved in some cases. Trypsin digest potently inhibited fusion. Negative controls included the reaction with VAMP4 + VAMP4 liposomes and VAMP4 liposomes with syntaxin 6 liposomes, which resulted in no fusion. However, trypsin digest of the VAMP4 + VAMP4 reaction did not result in fusion. Again, five of the seven reactions seem to induce fusion whereas the reactions sx13 + sx6-vti1a-VAMP4 as well as VAMP4-sx6 + sx13-vti1a did not show fusion. 


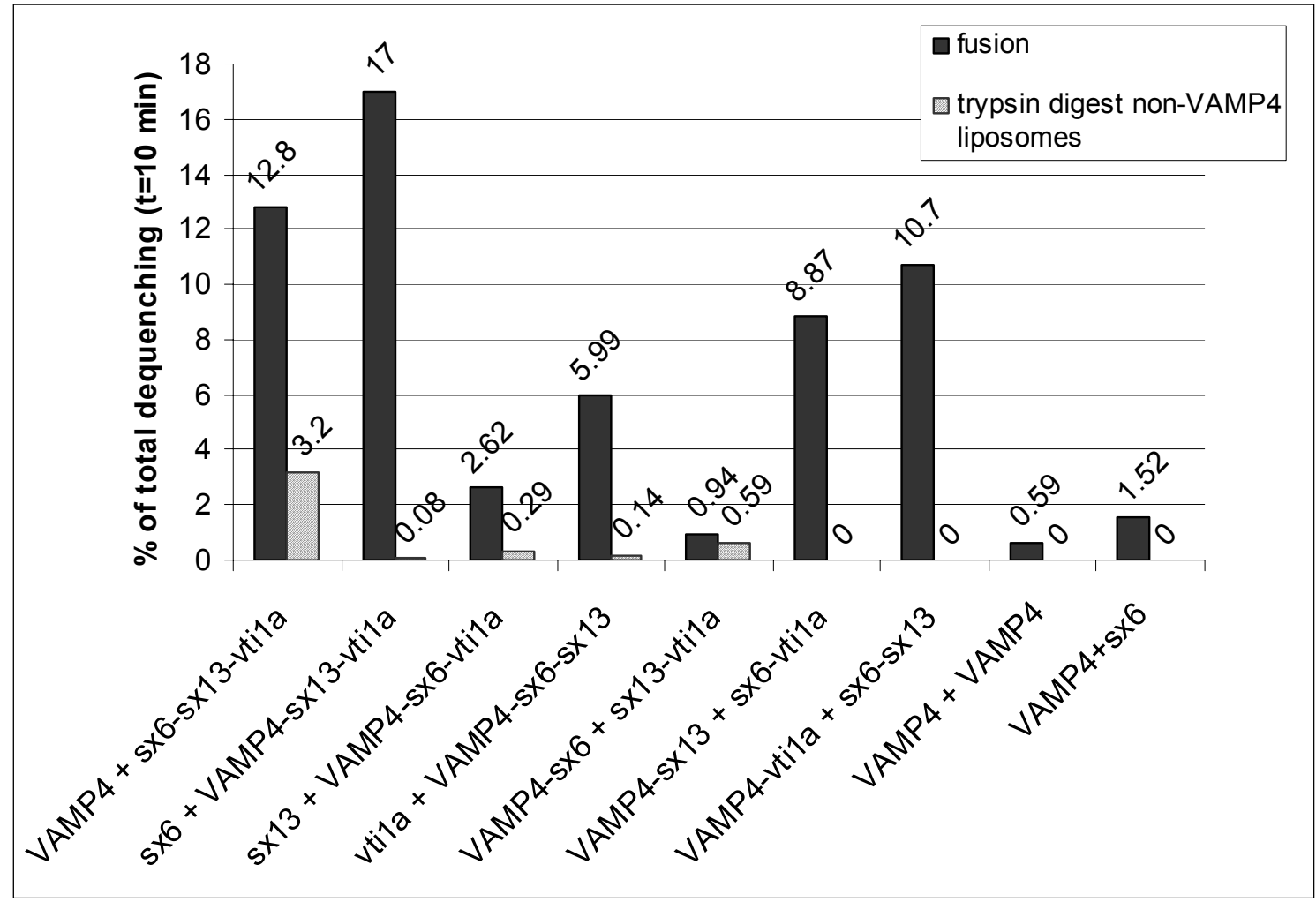

Figure 33 Bar-Diagram of NBD/Rho Dequenching Signals of all 7 Topological Combinations, Trypsinated Liposomes and Negative Controls. $5 \mu \mathrm{l}$ donor liposomes were combined with $35 \mu \mathrm{l}$

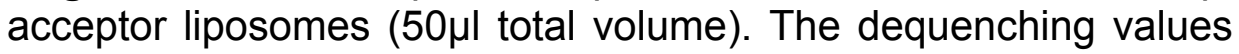
of the fusion reactions at $t=10 \mathrm{~min}$ are depicted as numbers on top of each bar. Solid bars, fusion; striped bars, one sided trypsin digest (digestion of the liposomes that did not contain VAMP4). The negative controls were performed with liposomes that contained only one SNARE protein each: VAMP4 or syntaxin 6 (including trypsin digest).

Five out of the seven reactions mediated fusion. The control experiments turned out to be difficult due to unexpected secondary effects of assembly kinetics of the cis-complexes, unspecific fusion signals of empty liposomes and trypsin digestion prevented 'straight forward' data interpretation. To verify the obtained results a different independent assay for liposomal fusion was used.

\subsubsection{Liposomal Fusion Observed by FRET Pairing of Labelled Lipids}

To further confirm the result obtained in the dequenching assay, the topological combinations were investigated using a different assay. In this FRET based assay flourescently labelled phosphatidylethanolamine (PE) was incorporated into the liposomes. One population contained Texas Red-PE and the other 
Oregon Green-PE. Oregon Green and Texas Red constitute a FRET (fluorescence resonance energy transfer) pair and upon lipid mixing of two liposomes FRET can be measured by excitation of Oregon Green and reading the emission wavelength of Texas Red (Figure 34). This assay offers an alternative to the dequenching assay because the FRET signal relies on the merger of different dyes in one liposome, making it almost the opposite of the dequenching assay. Moreover, there is no need for low donor to acceptor liposome ratios like in the dequenching assay. Equal amounts of the two liposome populations can be used.

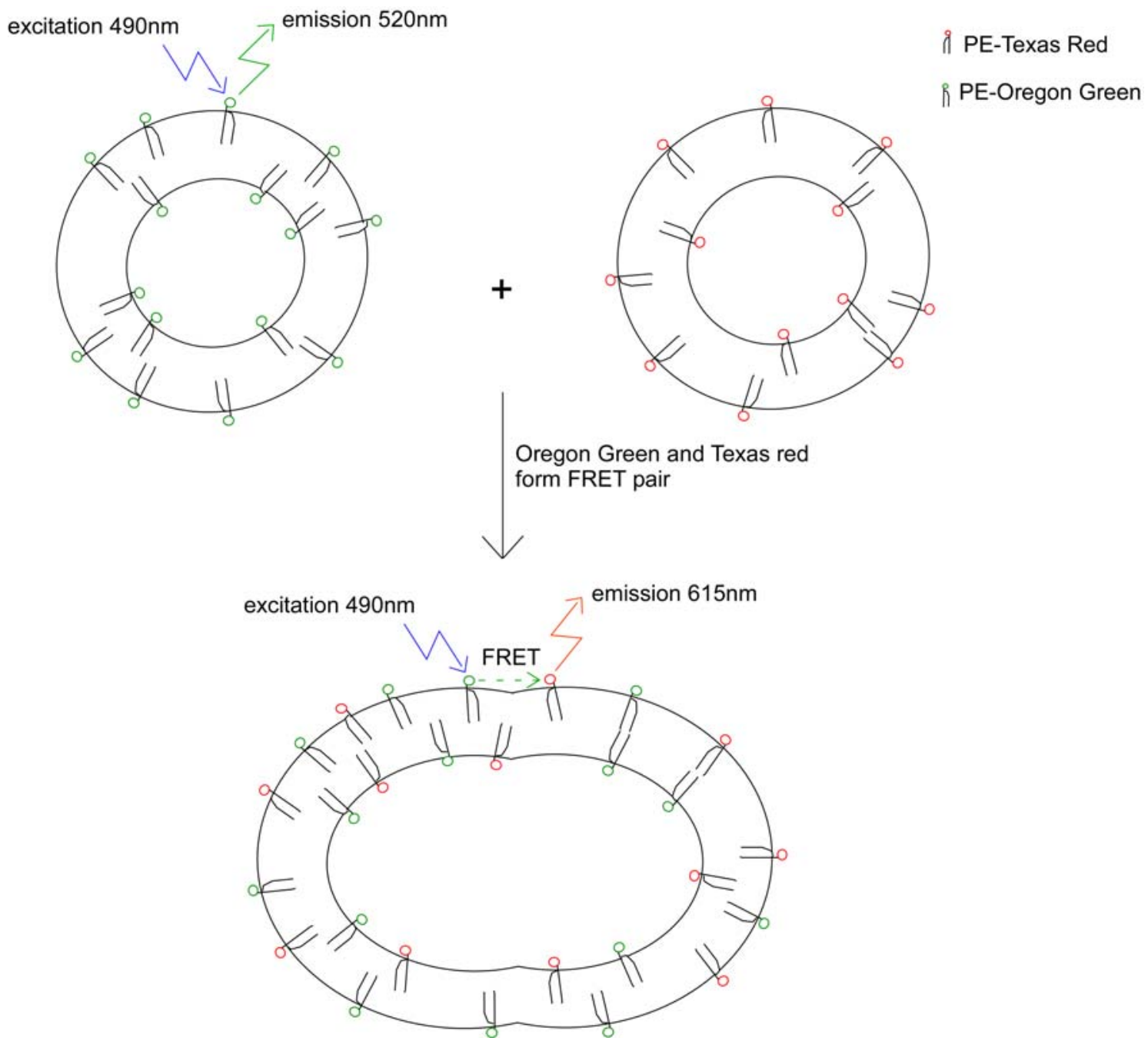

Figure 34 Principle of the Lipid FRET Assay. Oregon green and Texas red are separately inserted into two different liposome populations. Upon fusion the lipids mix in one membrane and form FRET pairs. Excitation of Oregon Green will lead to energy transfer to the Texas Red molecules. Thus, Texas Red emits light at its characteristic wavelength upon excitation of Oregon Green. FRET does not take place if the dyes are still separated in different membranes. 
The fusion curves and the respective 'one-sided' trypsin digests are shown for two example reactions (Figure 35).

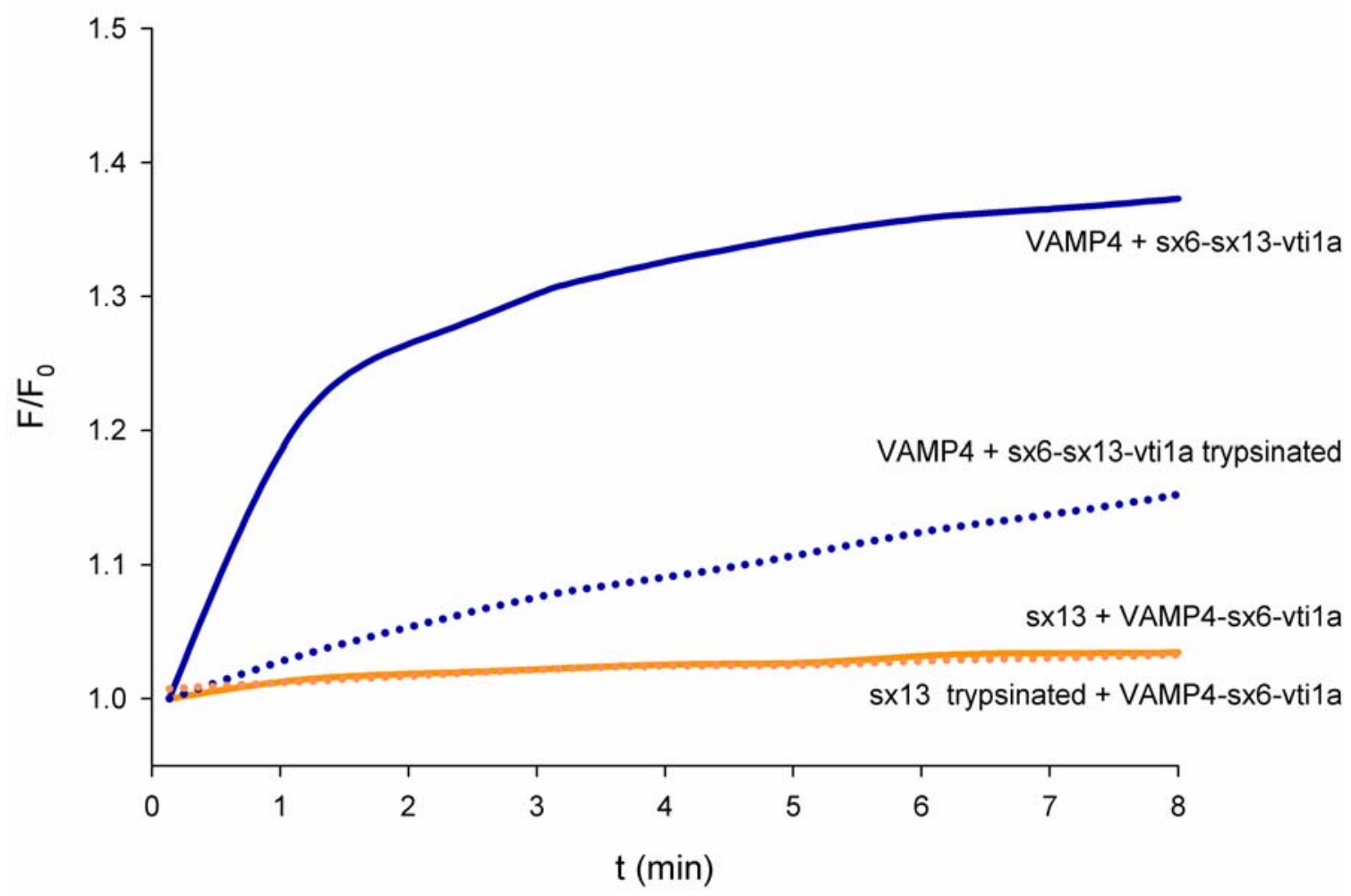

Figure 35 Fusion of Liposomes Monitored by Flourescently Labelled PE Lipids that Constitute a FRET Pair. Fusion can be monitored by incorporating Texas red and Oregon green labelled phosphatidylethanolamine in the two fusion partners. Upon merging of two differently labelled liposomes FRET can be measured by exciting Oregon green and measuring the Texas red emission. Fusion was inhibited by proteolytic digest of the liposomes that contained no VAMP4. The fluorescence signal was normalised to the starting signal. Fusion reactions, solid lines; trypsin digest, dotted lines.

The FRET signal increases rapidly within the first two to three minutes and then plateaus off quickly. One can explain the more rapid FRET kinetics when compared to the dequenching assay by the fact that the latter reports multiple rounds of fusion whereas the FRET assay does not. Once two differently labelled liposomes have merged, a FRET signal is generated. Upon fusion with a third liposome the signal does not increase much further, most likely because most dye molecules already have a FRET partner. In the dequenching assay, however, fusion with an additional unlabelled liposome results in further dilution 
of NBD and Rhodamine, thus increasing the NBD signal. Since the fluorescence signal increases fast and reaches the plateau quickly the initial rates of the fusion reactions were calculated rather than the plateau values to compare the reactions quantitatively (Figure 36 ). The initial rate was calculated by determination of the average slope of the graph in the first 120 seconds.

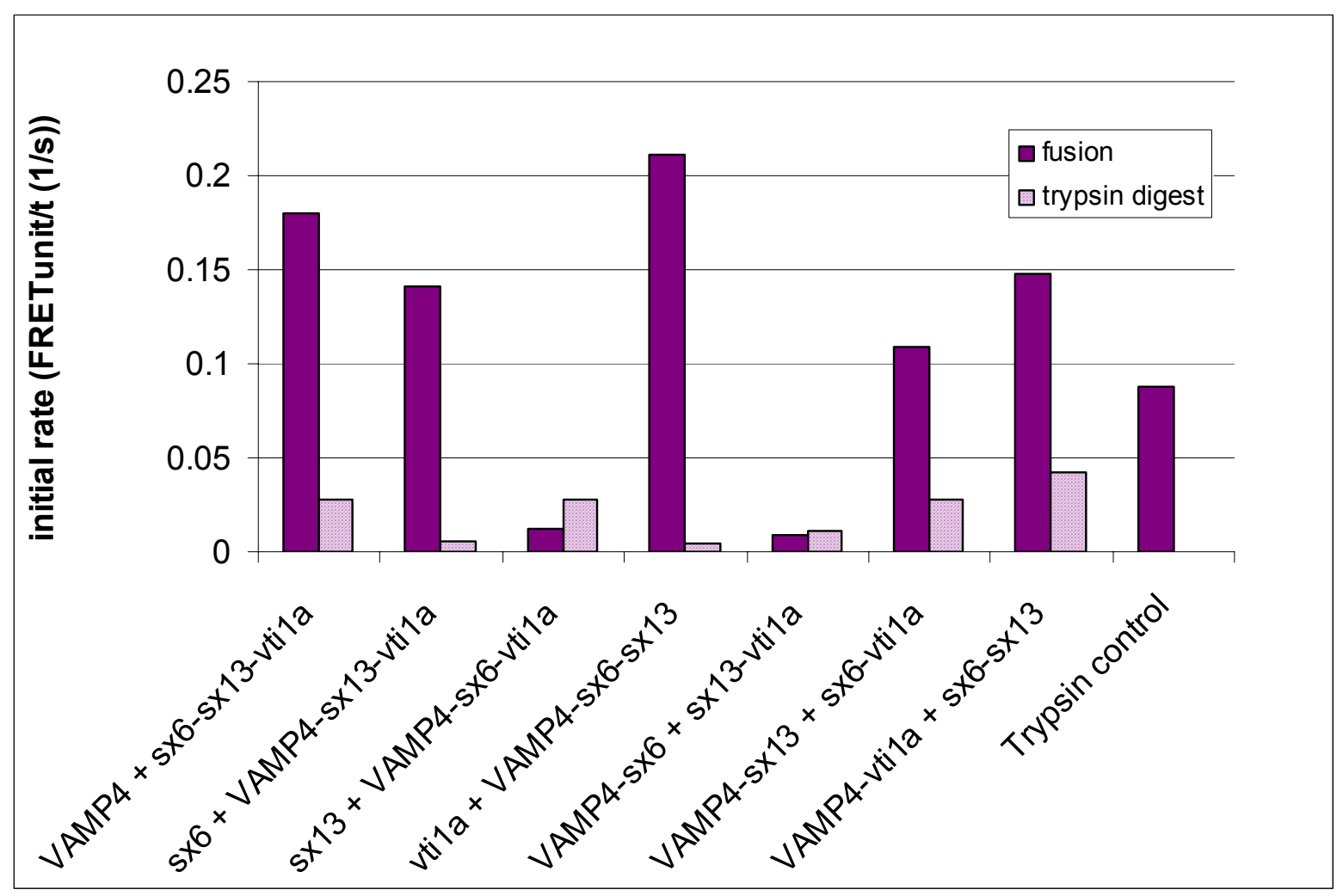

Figure 36 Bar Diagram of the Seven Topology Reactions Using the FRET Assay. The seven different topological combinations were tested for fusion using flourescently labelled lipids. FRET signal can be detected upon fusion of two different liposomes (purple bars). The trypsin digest knocks down fusion (striped bars). The trypsin control was performed by adding trypsin simultaneously, when mixing the liposomes for the fusion reaction. These data are derived from a representative experiment.

The five fusing reactions show high initial rates compared to the two non-fusing reactions. The Trypsin digestion knocked down fusion in all cases. In a control reaction trypsin was added simultaneously with the liposomes to check for nonspecific effects of trypsin. 


\begin{tabular}{|lcl|c|}
\hline \multicolumn{2}{|c|}{ Topological Combination } & Fusion efficiency \\
\hline VAMP4 & + & sx6-sx13-vti1a & ++ \\
\hline sx6 & + & VAMP4-sx13-vti1a & ++ \\
\hline sx13 & + & VAMP4-sx6-vti1a & - \\
\hline vti1a & + & VAMP4-sx6-sx13 & + \\
\hline VAMP4-sx6 & + & sx13-vti1a & - \\
\hline VAMP4-sx13 & + & sx6-vti1a & + \\
\hline VAMP4-vti1a & + & sx6-sx13 & ++ \\
\hline VAMP4 & + & VAMP4 & - \\
\hline VAMP4 & + & sx6 & - \\
\hline
\end{tabular}

Table 5 Summary of Fusion Effiencies of Topological Combinations. The results of all topology experiments were evaluated in a semiqualitative/quantitative manner and are summarised. ++, very good fusion efficiency; +, good fusion efficiency; -, no fusion.

Using two independent assays, (the dequenching assay and the FRET assay with flourescently labelled lipids) it was shown that the early endosomal SNAREs syntaxin 6 , syntaxin 13 , vti1a and VAMP4 were able fuse in more than one topological combination. Five combinations fused, whereas, two combinations did not (Table 5). These findings are contrary to the results of Parlati (Parlati et al., 2000). This group investigated the yeast ER-to-Golgi SNAREs and found only one combination that fused. For the early endosomal SNAREs it remains unclear why the addition of soluble SNARE fragments failed to inhibit the fusion reactions in some cases and why trypsin digest of both liposome populations shows remaining fusion activity. 


\section{Discussion}

\subsection{The Role of Syntaxin 6, Syntaxin 13, Syntaxin 16, Vti1a and VAMP4 in Early Endosomal Homotypic Fusion}

\subsubsection{Subcellular Organisation of the Early Endosomal SNAREs}

A detailed insight into the intracellular localisation and distribution of SNAREs is helpful to identify potential SNARE candidates and to characterise SNAREs functionally. It should be considered that SNAREs may have a broader distribution than expected. In order for a transport vesicle to fuse with an acceptor membrane, the correct SNARE proteins have to interact. After the fusion event the donor SNAREs have to be recycled back to the donor compartment while the acceptor SNAREs need to stay in the acceptor membrane. The SNAREs travel back via different transport steps and can be found on all intermediate membranes of the recycling pathway. In addition, due to incomplete sorting efficiency (or to one SNARE being involved in different fusion steps), there will always be overlap of the distribution of certain SNAREs over different neighbouring compartments. Syntaxin 1 and SNAP25 for example are mainly present on the plasma membrane but also in all intermediate organelles of vesicle recycling, the early endosome, and the synaptic vesicle (Otto et al., 1997). In addition, SNAREs with transmembrane domains are also found in the endoplasmic reticulum (ER), the Golgi apparatus and the transGolgi-network (TGN) since they travel trough these compartments in the course of the biosynthetic pathway, thus, proteins can be detected in organelles different from their main resident organelles. In order to identify potential candidates for a certain SNARE complex, one should also consider those proteins that are not selectively located on the respective organelle. In addition to subcellular localisation studies, coimmunoprecipitation and colocalisation studies should be performed to get insight into interactions between potential members of complexes.

The main place of action of SNARE proteins may differ from the main place of localisation. Syntaxin 8, for example, is localised mainly in the TGN and on early endosomes (Prekeris et al., 1999; Subramaniam et al., 2000) but Syntaxin 
8 is not involved in fusion steps of these organelles but in the homotypic fusion of late endosomes. As shown in this study, VAMP4, which is mostly present in the TGN but is also found on early endosomes, mediates early endosomal fusion as the R-SNARE. Syntaxin 6 is mainly located in TGN and endosomal compartments (Bock et al., 1997). Vti1a is mainly found in the TGN (Xu et al., 1998 ) and in nerve cells the splice variant vti1a- $\beta$ is mainly located in the synapse on small synaptic vesicles. Syntaxin 13 is the only SNARE that is mainly located on early endosomes (Prekeris et al., 1998).

\subsubsection{Function of the Early Endosomal SNAREs in fusion of PC12 early endosomes}

In this study (in collaboration with D. Brandhorst) competitive inhibition experiments with recombinant proteins were performed and $\mathrm{F}_{\mathrm{ab}}$-fragments were used to block fusion of PC12 early endosomes. Using the newly developed endosomal fusion assay (Brandhorst PhD thesis, 2004) a systematic analysis of all candidates was performed. All R-SNAREs and the candidates for the early endosomal SNAREs were screened. We identified syntaxin 6, syntaxin 13, vti1a and VAMP4 as the SNAREs mediating early endosomal homotypic fusion, since soluble fragments of these SNAREs exhibited the most potent inhibition. As controls, soluble domains of the neuronal SNAREs syntaxin 1, SNAP-25 and synaptobrevin, as well as the late endosomal SNAREs syntaxin 7, syntaxin 8, vti1 $\mathrm{b}$ and endobrevin were used and they appeared to have no effect (except a small effect with syntaxin 7). VAMP4 alone was as potent as the single QSNAREs. The fact that the four SNAREs syntaxin 6 , syntaxin 13 , vti1a and VAMP4, when used singly, had an equal effect on fusion might be an indication for a symmetrical distribution of all four SNAREs over the different early endosomes.

Syntaxin 16 was found in a complex with syntaxin 6, vti1a and VAMP4 which suggested that this may be the complex mediating early endosomal fusion (Kreykenbohm et al., 2002). However, the fact that syntaxin 16 in triple combination with syntaxin 6 and vti1a does not show strong inhibition of fusion in our assay does not support these earlier findings. Functional studies 
suggested the involvement of syntaxin 13 (in addition to syntaxin 6) in early endosomal fusion (Mills et al., 2001). Thus a functional role of syntaxin 13 as the $Q_{a}$-SNARE in early endosomal fusion is more likely. The same study showed inhibition of fusion using syntaxin 6 antibodies (Mills et al., 2001), supporting our findings. Syntaxin 16 and endobrevin, however, may have a minor role in this fusion step and it remains an open question whether they participate as substitutes for the SNAREs syntaxin 13 and VAMP4 or if they operate in parallel as parts of different complexes. The neuronal SNAREs are clearly not involved since Botulinum neurotoxin $E$, which specifically cleaves SNAP-25, has no effect on fusion of early endosomes (Brandhorst PhD thesis, 2004). Earlier, experiments using neurotoxins that specifically cleave syntaxin 1 and synaptobrevin (both being present on early endosomes) did not reveal inhibition of fusion (Link et al., 1993). Our experiments also confirmed the results of earlier studies for the involvement of vti1a and a minor role of endobrevin (Antonin et al., 2000b).

Taken together we identified the members of the SNARE complex that mediates the fusion of early endosomes.

\subsection{Biochemical and Structural Features of the Early Endosomal SNARE Complex}

\subsubsection{Biochemical and Biophysical Features}

The SNARE complex containing syntaxin 13, vti1a, syntaxin 6 and VAMP4 was assembled and characterised using biochemical and biophysical methods. The complex exhibited biochemical and structural features highly similar to the SNARE complexes previously described (Antonin et al., 2002b). The complex assembly rate was faster than the late endosomal complex but slower than the neuronal complex. Solubility was limited in buffers with less than $500 \mathrm{mM}$ salt. It is not clear why this complex was not soluble at lower salt concentrations. One reason might be the length of the syntaxin 13 core fragment. It is known from the neuronal complex that complex formation is impaired if the amino-terminal end is cut too close behind the SNARE motif (personal communication with D. Fasshauer). Here, syntaxin 13 contained four additional amino acids upstream 
of the -7 layer. However, experiments involving the full-length syntaxin 13, together with the other three SNAREs as core fragments, were not successful.

The monomeric core fragments of the early endosomal SNAREs are largely unstructured. As observed in other studies, ellipticity increased dramatically upon assembly of the monomers (Figure 9). This was found for the neuronal and the late endosomal complex using CD spectroscopy (Fasshauer et al., 1997). The complex is very thermostable, the melting point of $87^{\circ} \mathrm{C}$ being comparable to the $T_{m}$ of the neuronal complex (Fasshauer et al., 1999; Yang et al., 1999). In addition, the complex exhibited a pronounced hysteresis (Figure 13). The early endosomal complex had a disassembly and refolding behaviour which is more similar to that of the neuronal complex than to that of the late endosomal complex. Disassembly started at higher temperatures, and refolding occurred much faster and to a higher degree as compared to the late endosomal complex. Almost 90\% refolding occurred within 16h (Figure 12) whereas the endosomal complex exhibits only $60 \%$ refolding after 2 days (Fasshauer et al., 2002). Oligomerisation at lower salt concentrations was observed by MALLS (Figure 12). The early endosomal complex was monomeric in high salt buffer $(38 \mathrm{kDa}$ in $1 \mathrm{M} \mathrm{NaCl})$ and dimerised in low salt conditions $(60 \mathrm{kDa}$ in $500 \mathrm{mM} \mathrm{NaCl}$, Figure 12). Similar behaviour was observed for the late endosomal complex (Antonin et al., 2000a).

\subsubsection{Structural Features of the Early Endosomal SNARE Complex}

The crystal structure of the early endosomal complex was solved with a resolution of $2.7 \AA$. The structure is highly similar to that of the late endosomal and the neuronal SNARE complex (Antonin et al., 2002b). The positional assignments of the $Q_{a b c}$ and $R$-helices are identical (Figure 16). Several intraand intermolecular interactions were found and some of them are conserved among all three complexes (early and late endosomal and neuronal) whereas some are unique for the early endosomal complex. 
Layer +6 in the early endosomal complex is stabilised by hydrophobic interactions (Figure 19) as observed in the neuronal SNARE complex. In contrast, the late endosomal complex (and probably all complexes formed by the homologues of sx8 and vti1b) adapted the unusual glutamate in layer +6 . This glutamate is twisted to the outside and is stabilised by a conserved arginine in vti1b (R191, one position downstream of layer +6 ) (Antonin et al., 2002b).

Interestingly, Drosophila melanogaster syntaxin 8 also contains this conserved glutamate in layer +6 (Figure 37). It is not known how this residue is stabilised. In Drosophila only one vti1 isoform is expressed (no a/b isoforms). This vti1 must therefore be able to interact with both $Q_{c}$-SNAREs sx8 and sx6, assuming that complexes containing these combinations exist in Drosophila. The vti1 has an alanine in layer $+6(\mathrm{~A})$ followed by a downstream serine $(\underline{\mathrm{S}})$. This serine might interact with the polar threonine from sx6 if vti1a and sx6 form a complex (blue dotted line). Alternatively, the same serine residue might interact with the glutamate $(\underline{E})$ in layer +6 in syntaxin 8 (red dotted line, Figure 37). The glutamate might face the outside as was observed in the late endosomal complex and being stabilised by this interaction. Thus, it may be possible that Drosophila vti interacts with two isoforms of the $Q_{c}$-chain.

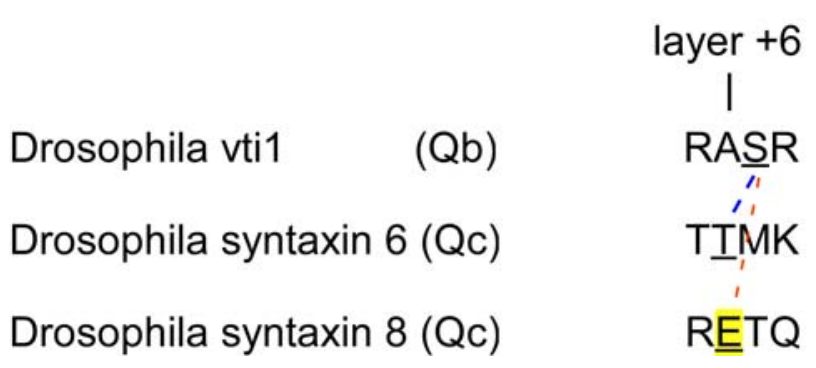

Figure 37 Possible Interactions Between Vti1 and Syntaxin 6 and Syntaxin 8 in Drosophila. Shown are parts of the sequences in layer +6 in the different proteins in Drosophila. Vti1 may bind syntaxin 6 via a serine threonine interaction (S-T, blue dotted line) or it may bind syntaxin 8 via a serine glutamate interaction (S-E, red dotted line). The unusual glutamate (E) may be stabilised by this interaction. 
Conserved residues are also the mediators in the interaction of vti1a with syntaxin 13. While the vti1b isoforms contain the conserved motif SIER, the sequence RLEA is conserved in the vti1a isoforms (Figure 20). In both cases the arginine is the interaction partner of a glutamate in the $Q_{a}-S N A R E$. Alternative intermolecular stabilisation mechanisms may have evolved (in vertebrates) where a mutation $(R \rightarrow A)$ demanded alternative modes of interaction.

The valine-glutamate-valine sandwich in Figure 21a shows an example of a surface interaction between the $Q_{b^{-}}$and $Q_{c}$-chain. The motif in vti1a, VETE, is conserved in the vti1a homologues. At the same time the motif SIGV is conserved in syntaxin 6 (the underlined residues are forming a salt bridge). The motifs of these cognate SNAREs complement each other. The same compatibility was discovered for the two SNAREs of the late endosomal complex, vti1b and syntaxin 8 (Figure 21b). This interaction could describe a phenomenon where cognate chains of complexes like vti1a and syntaxin 6 or vti $1 \mathrm{~b}$ and syntaxin 8 evolved in parallel. The chains within one pair maintained primary, secondary and tertiary protein structures that complement each other. As mentioned, Drosophila has only one vti1 protein. The conserved glutamate in the motif LETE is able to interact with both the serine in syntaxin 6 and the arginine in syntaxin 8 . Thus, it could form complexes with either one.

These results showed that SNARE complexes are highly similar in their structural features. Taking a closer look, however, reveals differences between the different complexes. Sequence alignments together with structural characterisations may give further insight into the nature of possible interactions of SNARE proteins from different subfamilies. For example, the presented analysis of vti1 in Drosophila demonstrated that vti1 is potentially able to interact with two different $\mathrm{Q}_{\mathrm{c}}$-chains. It remains to be shown if this is the case in vivo. 


\subsection{Topological Restriction of the Early Endosomal SNARE Complex in Liposomal Fusion}

In this study the restriction to certain topological combinations of the early endosomal SNAREs in liposomes was investigated. The experiments were performed using two independent assays that measure lipid mixing via either separation of two fluorescent dyes (dequenching) or combining dyes in one membrane after the fusion step (FRET). The data indicate that five of the seven investigated combinations resulted in fusion. Two combinations did not fuse, the combination in which the syntaxin $\left(Q_{a}\right)$ is alone in one liposome (sx13+sx6vti1a-VAMP4) and one 2:2 combination: VAMP4-sx13 + sx6-vti1a. It was previously shown that the yeast endoplasmic reticulum (ER) to Golgi SNAREs Bet1p and Bos1p, Sec22p, Sed5p only fuse in a $Q+R Q Q$ combination (Parlati et al., 2000). No other topological combination was found to fuse in this study. It was proposed that the so-called $1 \mathrm{v}$-SNARE to $3 \mathrm{t}$-SNARE distribution is the only combination in which fusion occurs. However, this was not observed with the early endosomal SNAREs.

The SNARE complex of the ER-to-Golgi transport is different from the early endosomal complex because it is composed of different SNAREs and it is mediating fusion of different membranes. The character of the directed ER-to Golgi transport is different from homotypic fusion of endosomes. ER-to-Golgi transport seems to be regulated by the asymmetric function of the SNAREs (Cao and Barlowe, 2000). The SNAREs on the ER-derived coat protein II (COPII) vesicles are characterised by a symmetric distribution but fusion requires a certain topological distribution (Cao and Barlowe, 2000). Bet1p and Bos $1 p$ are required on the vesicle and Sed5p activity is required on the target membrane. Interestingly the presence of Bos $1 p$ on the liposome together with Bet1p was not required in the liposomal assay (Parlati et al., 2000). A requirement of asymmetry for the early endosomal SNAREs in vivo is not known (Cao and Barlowe, 2000). However, our data using soluble SNARE fragments as inhibitors suggest that all four SNAREs are distributed in a symmetrical fashion on the endosomes (3.1.3) as neither of the single SNAREs was more powerful in inhibiting fusion than the others. 
Like the early endosomal complex, the late endosomal complex which includes syntaxin 7 , syntaxin 8 , vti1b and endobrevin, exhibits certain combinations that do not fuse (Zwilling Master Thesis, 2001). These are different from the ones used in the early endosomal complex. Late endosomal SNARE motifs attached to the transmembrane domains were incorporated into liposomes and the topological restrictions of fusion were investigated. Only the three combinations $\left(e b(R)+\right.$ sx7-sx8-vti1b, vti1b $\left(Q_{b}\right)+$ eb-sx7-sx8 and sx7 $\left(Q_{a}\right)+$ eb-sx8-vti1b) fused. No functioning 2:2 combination was observed. It is interesting that the combination $\operatorname{sx7}\left(Q_{a}\right)+$ eb-sx8-vti1b fuses while the $Q_{a}$-SNARE sx13 does not fuse in the corresponding combination with the early endosomal SNAREs. On the other hand sx6 $\left(Q_{c}\right)$ fuses with liposomes containing the remaining partners while the late endosomal counterpart of this reaction does not fuse. Taking into account the high structural conservation of the SNAREs it is surprising that just small differences in structure may result in such different kinetics.

Why do certain combinations induce fusion while others do not? One reason could be the formation of pre-complexes. From in vitro studies we know that pre-complexes of syntaxin 1 and SNAP-25 form (Fasshauer and Margittai, 2004). Liposomal fusion may be more efficient after these complexes have formed (Schuette et al., 2004). Three scenarios are possible. One is that certain combinations of SNAREs may form pre-complexes that act as acceptor complexes. These would enhance fusion efficiency. Alternatively, SNAREs may form non-productive binary or tertiary complexes and these 'dead end' complexes could prevent fusion. For the non-fusing combinations it is also possible that no pre-complexes form, thus decreasing the chance of full complex formation and fusion. No conclusion can be drawn from the $C D$ spectroscopy studies that were performed with double and triple combinations of the early endosomal SNAREs, because only the 1:1:1:1 combination resulted in complex formation. However, the CD studies were performed using only the SNARE motifs. The SNAREs studied are lacking their amino-terminal and transmembrane domains. Neither the amino-terminal domains nor the transmembrane domains were present because of the high background of ellipticity that these would have created. These domains, however, could be 
responsible for the formation of pre-complexes or the proper arrangement of SNAREs.

Fusion inhibition experiments were performed initially by adding soluble SNARE fragments to the liposomes as competitive inhibitors. Only the reaction $R+$ $Q Q Q$ was inhibited successfully. For the other combinations only partial inhibition was observed. The question arises why the cytosolic fragments SNAREs did not potently inhibit fusion in some instances. One reason might be the conformational state of the soluble SNAREs. Soluble SNAREs may be folded differently depending on the presence or absence of amino-terminal and transmembrane domains. The cytosolic fragments might have a conformational state that does not permit the formation of cis-complexes. Another reason might be that cis-complexes form but that these do not effectively inhibit fusion. The $\mathrm{CD}$ experiments using core fragments showed that the complex formation takes hours. If the formation of cis-complexes on liposomes using cytosolic fragments proceeds equally slowly then competitive inhibition may not be effective under the experimental conditions that were applied.

Initial inhibition experiments included proteolytic digestion of both liposome populations. This resulted in only an incomplete inhibition for some combinations. Digestion of one set of liposomes (the one that contained no VAMP4) was successful in inhibiting fusion completely. The proteins on the liposomes were always fully degraded as judged by SDS-PAGE (section 2.12, Figure 3). One reason why fusion still occurs could be that digestion of certain proteins results in fusogenic peptides. So far, no soluble peptide has been described in promoting fusion, however the transmembrane domains of synaptobrevin 2 and syntaxin 1 were described to be able to promote fusion, when inserted into liposomes (Langosch et al., 2001). The approach of digesting the 'non-VAMP4 liposomes' was successful. It may be possible that the digestion of VAMP4 with trypsin results in the generation of a fusogenic peptide. This may have been the transmembrane domain of VAMP4, though this needs to be further investigated. Interestingly, the digest of the negative control VAMP4 + VAMP4 did not show fusion, raising the question whether the 
digested VAMP4 alone promotes fusion or if this needs interaction partners coming from digested Q-SNAREs.

Topological restriction of SNAREs might be a regulatory element in heterotypic as well as in homotypic fusion of organelles. However, the results obtained in the liposomal fusion assay showed that for the early endosomal SNAREs fusion is possible in more combinations than reported for the ER-to-Golgi SNAREs. It remains to be clarified what determines the difference between the early endosomal, the late endosomal and the ER-to-Golgi SNAREs.

\subsection{Specificity of the Early Endosomal SNAREs in Vitro and in Vivo}

This study demonstrated that the four SNAREs syntaxin 6, syntaxin 13, vti1a and VAMP4 are sufficient to fuse liposomes. Strikingly, liposomes loaded with the Q-SNAREs are able to fuse with VAMP4 liposomes but also with liposomes containing the 'non-cognate' R-SNAREs endobrevin and synaptobrevin (Figure $26+27$ ). The fusion occurred with similar efficiencies as with the VAMP4 liposomes. Adding soluble VAMP4 fragments to the liposomes containing syntaxin 6, syntaxin 13 and vti1a resulted in inhibition of fusion with VAMP4 liposomes. The same fusion reaction was inhibited by adding soluble endobrevin or synaptobrevin. The $\mathrm{IC}_{50}$ values (Table 4 ) are within the same concentration range, thus the three different R-SNAREs are equally potent in inhibiting fusion. This indicates promiscuous behaviour and the formation of non-cognate SNARE complexes of the early endosomal Q-SNAREs with 'foreign' R-SNAREs. This is in contrast to the findings of the Rothman group (McNew et al., 2000). Thus, the data indicate that additional regulatory factors are responsible for the specificity observed in the fusion of early endosomes. Our results are supported by the observed promiscuous behaviour of SNAREs in vitro. Little discrimination was observed among SNAREs of the same subfamilies in their ability to form SNARE core complexes (Antonin et al., 2000a; Fasshauer et al., 1999; Yang et al., 1999). This supports the notion that mixed SNARE complexes form and are able to promote fusion at least in vitro. Further support was provided by the experiments performed in vivo (Bhattacharya et al., 2002; Holthuis et al., 1998; Liu and Barlowe, 2002) that 
demonstrate promiscuous behaviour of SNAREs. In addition, the deletion of several SNAREs including vti1b and endobrevin/VAMP8 resulted in surprisingly mild phenotypes, suggesting SNARE redundancy in the affected fusion step (Atlashkin et al., 2003).

In contrast, we observed a high degree of specificity in early endosomal fusion in the early endosomal fusion assay, which reflects more the in vivo situation. Screening all possible R-SNAREs and Q-SNAREs of three complexes revealed that only specific SNAREs inhibit fusion of early endosomes in the fusion assay. Whether non-cognate complexes form in vivo, is not known. However, soluble fragments of non-cognate R-SNAREs were not able to inhibit the fusion reaction in the in vitro endosomal fusion assay. Thus, we observe promiscuity in vitro while we observe a high degree of specificity in vivo.

It can be concluded that the early endosomal complex exhibits topological restriction that is different from the SNARE complexes studied so far. Features like topological restriction and compartmental specificity may not be the main regulatory mechanisms for these SNAREs. The mode and level of regulation might depend on the individual SNARE complex and the fusion step that is investigated. It is unlikely that efficient trafficking including specific membrane fusion relies mainly on the correct SNARE pairing. SNARE-mediated fusion is one of the last steps in a given trafficking process. Steps like directing the transport vesicle to the proper acceptor membrane and tethering must be specifically regulated in order to allow for efficient organelle transport. The interaction with (upstream) regulatory factors that ensure the correct tethering of organelles and formation of SNARE complexes must be the determinant for compartmental specificity in vivo. As outlined in section 1.1.2, regulatory proteins act in tethering by recruiting specific factors to the membrane. The assembly of multimeric protein complexes is necessary for tethering and these complexes also contain SM proteins that regulate SNARE activity. Recruitment of such regulatory factors to the endosome might be the reason for the observed specificity of SNAREs. These factors may be drawn from the rat brain cytosol that was added to the fusion reaction or residual contaminants from the PC12 endosome purification. However, earlier studies by another group showed 
that early endosomal fusion is abolished by leaving out cytosol. Fusion was restored to about $50 \%$ by adding EEA1 (Christoforidis et al., 1999a). This suggests that one important factor coming from the cytosol may be EEA1. Partial Rab5 effector complexes that mediate tethering in the in vitro assay may have assembled before purification of the endosomes. 


\section{Conclusions}

This study (in conjunction with the work of D. Brandhorst) demonstrated that the SNAREs syntaxin 6 , syntaxin 13 , vti1a and VAMP4 mediate the homotypic fusion of early endosomes. Liposomal fusion experiments showed that these proteins represent the minimal fusion machinery. The complex containing these SNAREs exhibited similar biochemical and biophysical features to the neuronal and late endosomal SNARE complexes. Analysis of the crystal structure revealed conserved, as well as alternative, interactions between the helices. Different R-SNAREs interacted promiscuously with the early endosomal QSNAREs. This contrasts with the findings in the early endosomal fusion assay where only VAMP4 (and to some extent endobrevin) inhibited fusion (Brandhorst PhD thesis, 2004). Furthermore, it was shown in two independent liposomal fusion assays that SNARE-mediated fusion involving these SNAREs was restricted to certain topological combinations: five out of seven possible combinations fused, two combinations did not. It remains to be investigated why the early endosomal SNAREs exhibited promiscuity in the liposomal fusion assay while they behaved in a specific manner in the endosomal fusion assay. It also remains to be investigated why the ER-to-Golgi SNAREs fuse in only one topological combination while the early endosomal SNAREs are able to fuse in five combinations. Future experiments will have to be performed to shed more light on the regulation of SNAREs in vivo and in vitro. 


\section{Appendix}

Table 6 Crystallographic Data

\begin{tabular}{|c|c|}
\hline Data collection & \\
\hline Space group & $\mathrm{C} 2$ \\
\hline Unit cell $\left(\AA{ }^{\circ},{ }^{\circ}\right)$ & \\
\hline a & 252.9 \\
\hline b & 28.7 \\
\hline c & 41.9 \\
\hline$\beta$ & 98.2 \\
\hline Wavelength $(\AA)$ & 0.90 \\
\hline Resolution $(\AA)$ & $30.0-2.5$ \\
\hline Unique reflections & \\
\hline Total & 50005 \\
\hline Unique & 9365 \\
\hline Redundancy & 5.3 \\
\hline Redundancy & 2.7 \\
\hline Completeness (\%) & $87.2(43.4)$ \\
\hline $1 / \sigma(I)$ & $8.7(1.9)$ \\
\hline $\mathrm{R}_{\text {sym }}{ }^{\mathrm{a}}(\%)$ & \\
\hline Crystal 1 & 5.4 \\
\hline Crystal 2 & 3.9 \\
\hline Crystal 3 & 5.0 \\
\hline Crystal 4 & 5.2 \\
\hline Crystal 5 & 11.8 \\
\hline$R_{\text {merge }}{ }^{a}(\%)$ & $12.4(49.0)$ \\
\hline Refinement & \\
\hline Resolution $(\AA)$ & $30.0-2.5$ \\
\hline Model atoms & \\
\hline Protein & 2168 \\
\hline Water oxygens & 49 \\
\hline $\mathrm{R}_{\text {work }}^{\mathrm{b}}(\%)$ & 25.2 \\
\hline $\mathrm{R}_{\text {free }}{ }^{\mathrm{b}}(\%)$ & 29.6 \\
\hline RMSD $^{\mathrm{e}}$ from ideality & \\
\hline Bond lengths $(\AA)$ & 0.007 \\
\hline Bond angles $\left({ }^{\circ}\right)$ & 1.00 \\
\hline Bonded B-factors $\left(\AA^{2}\right)$ & \\
\hline Main chain bonds & 1.4 \\
\hline Side chain bonds & 2.6 \\
\hline Main chain angles & 2.4 \\
\hline Side chain angles & 4.1 \\
\hline B-factors $\left(\AA^{2}\right)$ & \\
\hline Protein & 65.6 \\
\hline Water & 60.6 \\
\hline Wilson & 68.5 \\
\hline$\phi / \psi(\%)$ & \\
\hline Core & 96.4 \\
\hline Addionally allowed & 3.2 \\
\hline Generally allowed & 0 \\
\hline Disallowed & 0.4 \\
\hline
\end{tabular}

Values for the last $0.1 \AA$ in parentheses

${ }^{a} R_{\text {sym }}(I)=\left(\Sigma_{\mathrm{hkl}} \Sigma_{i}\left[\left|l_{\mathrm{i}}(\mathrm{hkl})-<l(\mathrm{hkl})>\right|\right] / \Sigma_{\mathrm{hk}} \Sigma_{\mathrm{i}} l_{\mathrm{i}}(\mathrm{hkl})\right] ; l_{\mathrm{i}}(\mathrm{hkl})$ - intensity of the $\mathrm{i}^{\text {th }}$ measurement of $\mathrm{hkl} ;<$ (hkl) $>-$ average value of hkl for all i measurements; $R_{\text {merge }}-$ over all five crystals

${ }^{\mathrm{b}} \mathrm{R}_{\text {work }}=\Sigma_{\text {hkl }}\left[|| \mathrm{F}_{\text {obs }}|-k| \mathrm{F}_{\text {calc }}||\right] / \Sigma_{\mathrm{hkl}}\left[\left|\mathrm{F}_{\text {obs }}\right|\right] ; \mathrm{R}_{\text {free }}=\Sigma_{\text {hklcT }}\left[|| \mathrm{F}_{\text {obs }}|-k| \mathrm{F}_{\text {calc }}||\right] / \Sigma_{\text {hkl cT }}\left[\left|F_{\text {obs }}\right|\right]$; hklсT - test set. 


\section{$7 \quad$ Bibliography}

(1994) The CCP4 suite: programs for protein crystallography. Acta Crystallogr D Biol Crystallogr, 50, 760-763.

Abazeed, M.E., Blanchette, J.M. and Fuller, R.S. (2005) Cell-free transport from the trans-golgi network to late endosome requires factors involved in formation and consumption of clathrin-coated vesicles. J Biol Chem, 280, 4442-4450.

Advani, R.J., Yang, B., Prekeris, R., Lee, K.C., Klumperman, J. and Scheller, R.H. (1999) VAMP-7 mediates vesicular transport from endosomes to lysosomes. J Cell Biol, 146, 765-776.

Aniento, F., Emans, N., Griffiths, G. and Gruenberg, J. (1993) Cytoplasmic dynein-dependent vesicular transport from early to late endosomes. $J$ Cell Biol, 123, 1373-1387.

Antonin, W., Dulubova, I., Arac, D., Pabst, S., Plitzner, J., Rizo, J. and Jahn, R. (2002a) The N-terminal domains of syntaxin 7 and vti1b form three-helix bundles that differ in their ability to regulate SNARE complex assembly. $J$ Biol Chem, 277, 36449-36456.

Antonin, W., Fasshauer, D., Becker, S., Jahn, R. and Schneider, T.R. (2002b) Crystal structure of the endosomal SNARE complex reveals common structural principles of all SNAREs. Nat Struct Biol, 9, 107-111.

Antonin, W., Holroyd, C., Fasshauer, D., Pabst, S., Von Mollard, G.F. and Jahn, R. (2000a) A SNARE complex mediating fusion of late endosomes defines conserved properties of SNARE structure and function. Embo J, 19, 6453-6464.

Antonin, W., Holroyd, C., Tikkanen, R., Honing, S. and Jahn, R. (2000b) The RSNARE endobrevin/VAMP-8 mediates homotypic fusion of early endosomes and late endosomes. Mol Biol Cell, 11, 3289-3298.

Antonin, W., Riedel, D. and von Mollard, G.F. (2000c) The SNARE Vti1a-beta is localized to small synaptic vesicles and participates in a novel SNARE complex. J Neurosci, 20, 5724-5732.

Atlashkin, V., Kreykenbohm, V., Eskelinen, E.L., Wenzel, D., Fayyazi, A. and Fischer von Mollard, G. (2003) Deletion of the SNARE vti1b in mice results in the loss of a single SNARE partner, syntaxin 8. Mol Cell Biol, 23, 5198-5207.

Barbieri, M.A., Hoffenberg, S., Roberts, R., Mukhopadhyay, A., Pomrehn, A., Dickey, B.F. and Stahl, P.D. (1998) Evidence for a symmetrical requirement for Rab5-GTP in in vitro endosome-endosome fusion. $\mathrm{J}$ Biol Chem, 273, 25850-25855.

Bhattacharya, S., Stewart, B.A., Niemeyer, B.A., Burgess, R.W., McCabe, B.D., Lin, P., Boulianne, G., O'Kane, C.J. and Schwarz, T.L. (2002) Members of the synaptobrevin/vesicle-associated membrane protein (VAMP) family in Drosophila are functionally interchangeable in vivo for neurotransmitter release and cell viability. Proc Natl Acad Sci U S A, 99, $13867-13872$.

Blanchette, J.M., Abazeed, M.E. and Fuller, R.S. (2004) Cell-free reconstitution of transport from the trans-golgi network to the late endosome/prevacuolar compartment. J Biol Chem, 279, 48767-48773. 
Block, M.R., Glick, B.S., Wilcox, C.A., Wieland, F.T. and Rothman, J.E. (1988) Purification of an $\mathrm{N}$-ethylmaleimide-sensitive protein catalyzing vesicular transport. Proc Natl Acad Sci U S A, 85, 7852-7856.

Bock, J.B., Klumperman, J., Davanger, S. and Scheller, R.H. (1997) Syntaxin 6 functions in trans-Golgi network vesicle trafficking. Mol Biol Cell, 8, 12611271.

Bock, J.B., Lin, R.C. and Scheller, R.H. (1996) A new syntaxin family member implicated in targeting of intracellular transport vesicles. J Biol Chem, 271, 17961-17965.

Bock, J.B., Matern, H.T., Peden, A.A. and Scheller, R.H. (2001) A genomic perspective on membrane compartment organization. Nature, 409, 839841.

Boeddinghaus, C., Merz, A.J., Laage, R. and Ungermann, C. (2002) A cycle of Vam7p release from and Ptdlns 3-P-dependent rebinding to the yeast vacuole is required for homotypic vacuole fusion. J Cell Biol, 157, 79-89.

Borisovska, M., Zhao, Y., Tsytsyura, Y., Glyvuk, N., Takamori, S., Matti, U., Rettig, J., Sudhof, T. and Bruns, D. (2005) v-SNAREs control exocytosis of vesicles from priming to fusion. Embo J, 24, 2114-2126.

Bradford, M.M. (1976) A rapid and sensitive method for the quantitation of microgram quantities of protein utilizing the principle of protein-dye binding. Anal Biochem, 72, 248-254.

Braell, W.A. (1987) Fusion between endocytic vesicles in a cell-free system. Proc Natl Acad Sci U S A, 84, 1137-1141.

Brandhorst PhD thesis, D. (2004) Entwicklung eines in vitro Fluoreszenzassays zur Charakterisierung der Fusion von frühen Endosomen.

Brunger, A.T., Adams, P.D., Clore, G.M., DeLano, W.L., Gros, P., GrosseKunstleve, R.W., Jiang, J.S., Kuszewski, J., Nilges, M., Pannu, N.S., Read, R.J., Rice, L.M., Simonson, T. and Warren, G.L. (1998) Crystallography \& NMR system: A new software suite for macromolecular structure determination. Acta Crystallogr D Biol Crystallogr, 54 ( Pt 5), 905-921.

Bryant, N.J. and James, D.E. (2001) Vps45p stabilizes the syntaxin homologue TIg2p and positively regulates SNARE complex formation. Embo J, 20, 3380-3388.

Bucci, C., Parton, R.G., Mather, I.H., Stunnenberg, H., Simons, K., Hoflack, B. and Zerial, M. (1992) The small GTPase rab5 functions as a regulatory factor in the early endocytic pathway. Cell, 70, 715-728.

Cao, X. and Barlowe, C. (2000) Asymmetric requirements for a Rab GTPase and SNARE proteins in fusion of COPII vesicles with acceptor membranes. J Cell Biol, 149, 55-66.

Ceccarelli, B., Hurlbut, W.P. and Mauro, A. (1973) Turnover of transmitter and synaptic vesicles at the frog neuromuscular junction. J Cell Biol, 57, 499524.

Cheever, M.L., Sato, T.K., de Beer, T., Kutateladze, T.G., Emr, S.D. and Overduin, M. (2001) Phox domain interaction with Ptdlns(3)P targets the Vam7 t-SNARE to vacuole membranes. Nat Cell Biol, 3, 613-618.

Christoforidis, S., McBride, H.M., Burgoyne, R.D. and Zerial, M. (1999a) The Rab5 effector EEA1 is a core component of endosome docking. Nature, 397, 621-625.

Christoforidis, S., Miaczynska, M., Ashman, K., Wilm, M., Zhao, L., Yip, S.C., Waterfield, M.D., Backer, J.M. and Zerial, M. (1999b) 
Phosphatidylinositol-3-OH kinases are Rab5 effectors. Nat Cell Biol, 1, 249-252.

Clary, D.O., Griff, I.C. and Rothman, J.E. (1990) SNAPs, a family of NSF attachment proteins involved in intracellular membrane fusion in animals and yeast. Cell, 61, 709-721.

Conradt, B., Haas, A. and Wickner, W. (1994) Determination of four biochemically distinct, sequential stages during vacuole inheritance in vitro. J Cell Biol, 126, 99-110.

Cook, N.R., Row, P.E. and Davidson, H.W. (2004) Lysosome associated membrane protein 1 (Lamp1) traffics directly from the TGN to early endosomes. Traffic, 5, 685-699.

Darsow, T., Rieder, S.E. and Emr, S.D. (1997) A multispecificity syntaxin homologue, Vam3p, essential for autophagic and biosynthetic protein transport to the vacuole. J Cell Biol, 138, 517-529.

De Camilli, P. and Takei, K. (1996) Molecular mechanisms in synaptic vesicle endocytosis and recycling. Neuron, 16, 481-486.

Diaz, R., Mayorga, L. and Stahl, P. (1988) In vitro fusion of endosomes following receptor-mediated endocytosis. J Biol Chem, 263, 6093-6100.

Dulubova, I., Sugita, S., Hill, S., Hosaka, M., Fernandez, I., Sudhof, T.C. and Rizo, J. (1999) A conformational switch in syntaxin during exocytosis: role of munc18. Embo J, 18, 4372-4382.

Dulubova, I., Yamaguchi, T., Gao, Y., Min, S.W., Huryeva, I., Sudhof, T.C. and Rizo, J. (2002) How Tlg2p/syntaxin 16 'snares' Vps45. Embo J, 21, 36203631.

Dunn, K.W. and Maxfield, F.R. (1992) Delivery of ligands from sorting endosomes to late endosomes occurs by maturation of sorting endosomes. J Cell Biol, 117, 301-310.

Eitzen, G., Thorngren, N. and Wickner, W. (2001) Rho1p and Cdc42p act after Ypt7p to regulate vacuole docking. Embo J, 20, 5650-5656.

Ernst, J.A. and Brunger, A.T. (2003) High resolution structure, stability, and synaptotagmin binding of a truncated neuronal SNARE complex. J Biol Chem, 278, 8630-8636.

Fasshauer, D., Antonin, W., Margittai, M., Pabst, S. and Jahn, R. (1999) Mixed and non-cognate SNARE complexes. Characterization of assembly and biophysical properties. J Biol Chem, 274, 15440-15446.

Fasshauer, D., Antonin, W., Subramaniam, V. and Jahn, R. (2002) SNARE assembly and disassembly exhibit a pronounced hysteresis. Nat Struct Biol, 9, 144-151.

Fasshauer, D., Eliason, W.K., Brunger, A.T. and Jahn, R. (1998a) Identification of a minimal core of the synaptic SNARE complex sufficient for reversible assembly and disassembly. Biochemistry, 37, 10354-10362.

Fasshauer, D. and Margittai, M. (2004) A transient N-terminal interaction of SNAP-25 and syntaxin nucleates SNARE assembly. J Biol Chem, 279, 7613-7621.

Fasshauer, D., Otto, H., Eliason, W.K., Jahn, R. and Brunger, A.T. (1997) Structural changes are associated with soluble $\mathrm{N}$-ethylmaleimidesensitive fusion protein attachment protein receptor complex formation. $J$ Biol Chem, 272, 28036-28041.

Fasshauer, D., Sutton, R.B., Brunger, A.T. and Jahn, R. (1998b) Conserved structural features of the synaptic fusion complex: SNARE proteins 
reclassified as Q- and R-SNAREs. Proc Natl Acad Sci U S A, 95, 1578115786.

Ferro-Novick, S. and Jahn, R. (1994) Vesicle fusion from yeast to man. Nature, 370, 191-193.

Fiebig, K.M., Rice, L.M., Pollock, E. and Brunger, A.T. (1999) Folding intermediates of SNARE complex assembly. Nat Struct Biol, 6, 117-123.

Fukasawa, M., Varlamov, O., Eng, W.S., Sollner, T.H. and Rothman, J.E. (2004) Localization and activity of the SNARE Ykt6 determined by its regulatory domain and palmitoylation. Proc Natl Acad Sci U S A, 101, 4815-4820.

Gillooly, D.J., Simonsen, A. and Stenmark, H. (2001) Cellular functions of phosphatidylinositol 3-phosphate and FYVE domain proteins. Biochem J, 355, 249-258.

Gorvel, J.P., Chavrier, P., Zerial, M. and Gruenberg, J. (1991) rab5 controls early endosome fusion in vitro. Cell, 64, 915-925.

Gotte, M. and Gallwitz, D. (1997) High expression of the yeast syntaxin-related Vam3 protein suppresses the protein transport defects of a pep12 null mutant. FEBS Lett, 411, 48-52.

Graf, C.T., Riedel, D., Schmitt, H.D. and Jahn, R. (2005) Identification of functionally interacting SNAREs by using complementary substitutions in the conserved '0' layer. Mol Biol Cell, 16, 2263-2274.

Gruenberg, J., Griffiths, G. and Howell, K.E. (1989) Characterization of the early endosome and putative endocytic carrier vesicles in vivo and with an assay of vesicle fusion in vitro. J Cell Biol, 108, 1301-1316.

Gruenberg, J. and Howell, K.E. (1987) An internalized transmembrane protein resides in a fusion-competent endosome for less than 5 minutes. Proc Natl Acad Sci U S A, 84, 5758-5762.

Guo, W., Sacher, M., Barrowman, J., Ferro-Novick, S. and Novick, P. (2000) Protein complexes in transport vesicle targeting. Trends Cell Biol, 10, 251-255.

Gurunathan, S., Chapman-Shimshoni, D., Trajkovic, S. and Gerst, J.E. (2000) Yeast exocytic v-SNAREs confer endocytosis. Mol Biol Cell, 11, 36293643.

Hatsuzawa, K., Lang, T., Fasshauer, D., Bruns, D. and Jahn, R. (2003) The RSNARE motif of tomosyn forms SNARE core complexes with syntaxin 1 and SNAP-25 and down-regulates exocytosis. J Biol Chem, 278, 3115931166.

Hayashi, T., McMahon, H., Yamasaki, S., Binz, T., Hata, Y., Sudhof, T.C. and Niemann, H. (1994) Synaptic vesicle membrane fusion complex: action of clostridial neurotoxins on assembly. Embo J, 13, 5051-5061.

Hayashi, T., Yamasaki, S., Nauenburg, S., Binz, T. and Niemann, H. (1995) Disassembly of the reconstituted synaptic vesicle membrane fusion complex in vitro. Embo J, 14, 2317-2325.

Hermann, G.J., Thatcher, J.W., Mills, J.P., Hales, K.G., Fuller, M.T., Nunnari, J. and Shaw, J.M. (1998) Mitochondrial fusion in yeast requires the transmembrane GTPase Fzo1p. J Cell Biol, 143, 359-373.

Hess, D.T., Slater, T.M., Wilson, M.C. and Skene, J.H. (1992) The 25 kDa synaptosomal-associated protein SNAP-25 is the major methionine-rich polypeptide in rapid axonal transport and a major substrate for palmitoylation in adult CNS. J Neurosci, 12, 4634-4641. 
Heuser, J.E. and Reese, T.S. (1973) Evidence for recycling of synaptic vesicle membrane during transmitter release at the frog neuromuscular junction. J Cell Biol, 57, 315-344.

Holroyd, C., Kistner, U., Annaert, W. and Jahn, R. (1999) Fusion of endosomes involved in synaptic vesicle recycling. Mol Biol Cell, 10, 3035-3044.

Holthuis, J.C., Nichols, B.J. and Pelham, H.R. (1998) The syntaxin Tlg1p mediates trafficking of chitin synthase III to polarized growth sites in yeast. Mol Biol Cell, 9, 3383-3397.

Horiuchi, H., Lippe, R., McBride, H.M., Rubino, M., Woodman, P., Stenmark, H., Rybin, V., Wilm, M., Ashman, K., Mann, M. and Zerial, M. (1997) A novel Rab5 GDP/GTP exchange factor complexed to Rabaptin-5 links nucleotide exchange to effector recruitment and function. Cell, 90, 11491159.

Itin, C., Rancano, C., Nakajima, Y. and Pfeffer, S.R. (1997) A novel assay reveals a role for soluble $\mathrm{N}$-ethylmaleimide-sensitive fusion attachment protein in mannose 6-phosphate receptor transport from endosomes to the trans Golgi network. J Biol Chem, 272, 27737-27744.

Jahn, R., Lang, T. and Sudhof, T.C. (2003) Membrane fusion. Cell, 112, 519533.

Jahn, R. and Niemann, H. (1994) Molecular mechanisms of clostridial neurotoxins. Ann N Y Acad Sci, 733, 245-255.

Jahn, R. and Sudhof, T.C. (1999) Membrane fusion and exocytosis. Annu Rev Biochem, 68, 863-911.

Katz, L. and Brennwald, P. (2000) Testing the 3Q:1R "rule": mutational analysis of the ionic "zero" layer in the yeast exocytic SNARE complex reveals no requirement for arginine. Mol Biol Cell, 11, 3849-3858.

Kreykenbohm, V., Wenzel, D., Antonin, W., Atlachkine, V. and von Mollard, G.F. (2002) The SNAREs vti1a and vti1b have distinct localization and SNARE complex partners. Eur J Cell Biol, 81, 273-280.

Langosch, D., Crane, J.M., Brosig, B., Hellwig, A., Tamm, L.K. and Reed, J. (2001) Peptide mimics of SNARE transmembrane segments drive membrane fusion depending on their conformational plasticity. $\mathrm{J} \mathrm{Mol} \mathrm{Biol,}$ 311, 709-721.

Lewis, M.J., Nichols, B.J., Prescianotto-Baschong, C., Riezman, H. and Pelham, H.R. (2000) Specific retrieval of the exocytic SNARE Snc1p from early yeast endosomes. Mol Biol Cell, 11, 23-38.

Link, E., McMahon, H., Fischer von Mollard, G., Yamasaki, S., Niemann, H., Sudhof, T.C. and Jahn, R. (1993) Cleavage of cellubrevin by tetanus toxin does not affect fusion of early endosomes. J Biol Chem, 268, $18423-18426$.

Liu, Y. and Barlowe, C. (2002) Analysis of Sec22p in endoplasmic reticulum/Golgi transport reveals cellular redundancy in SNARE protein function. Mol Biol Cell, 13, 3314-3324.

Luzio, J.P., Rous, B.A., Bright, N.A., Pryor, P.R., Mullock, B.M. and Piper, R.C. (2000) Lysosome-endosome fusion and lysosome biogenesis. J Cell Sci, 113 ( Pt 9), 1515-1524.

Maxfield, F.R. and McGraw, T.E. (2004) Endocytic recycling. Nat Rev Mol Cell Biol, 5, 121-132.

Mayer, A. and Wickner, W. (1997) Docking of yeast vacuoles is catalyzed by the Ras-like GTPase Ypt7p after symmetric priming by Sec18p (NSF). J Cell Biol, 136, 307-317. 
Mayer, A., Wickner, W. and Haas, A. (1996) Sec18p (NSF)-driven release of Sec17p (alpha-SNAP) can precede docking and fusion of yeast vacuoles. Cell, 85, 83-94.

Mayorga, L.S., Beron, W., Sarrouf, M.N., Colombo, M.I., Creutz, C. and Stahl, P.D. (1994) Calcium-dependent fusion among endosomes. J Biol Chem, 269, 30927-30934.

Mayorga, L.S., Diaz, R. and Stahl, P.D. (1988) Plasma membrane-derived vesicles containing receptor-ligand complexes are fusogenic with early endosomes in a cell-free system. J Biol Chem, 263, 17213-17216.

McBride, H.M., Rybin, V., Murphy, C., Giner, A., Teasdale, R. and Zerial, M. (1999) Oligomeric complexes link Rab5 effectors with NSF and drive membrane fusion via interactions between EEA1 and syntaxin 13. Cell, 98, 377-386.

McNew, J.A., Parlati, F., Fukuda, R., Johnston, R.J., Paz, K., Paumet, F., Sollner, T.H. and Rothman, J.E. (2000) Compartmental specificity of cellular membrane fusion encoded in SNARE proteins. Nature, 407, 153159.

Miller, T.M. and Heuser, J.E. (1984) Endocytosis of synaptic vesicle membrane at the frog neuromuscular junction. J Cell Biol, 98, 685-698.

Mills, I.G., Urbe, S. and Clague, M.J. (2001) Relationships between EEA1 binding partners and their role in endosome fusion. J Cell Sci, 114, 19591965.

Montecucco, C. and Schiavo, G. (1994) Mechanism of action of tetanus and botulinum neurotoxins. Mol Microbiol, 13, 1-8.

Mukherjee, S., Ghosh, R.N. and Maxfield, F.R. (1997) Endocytosis. Physiol Rev, 77, 759-803.

Muller, O., Johnson, D.I. and Mayer, A. (2001) Cdc42p functions at the docking stage of yeast vacuole membrane fusion. Embo J, 20, 5657-5665.

Munn, A.L. (2000) The yeast endocytic membrane transport system. Microsc Res Tech, 51, 547-562.

Munson, M., Chen, X., Cocina, A.E., Schultz, S.M. and Hughson, F.M. (2000) Interactions within the yeast t-SNARE Sso1p that control SNARE complex assembly. Nat Struct Biol, 7, 894-902.

Murray, J.T., Panaretou, C., Stenmark, H., Miaczynska, M. and Backer, J.M. (2002) Role of Rab5 in the recruitment of hVps34/p150 to the early endosome. Traffic, 3, 416-427.

Nichols, B.J., Ungermann, C., Pelham, H.R., Wickner, W.T. and Haas, A. (1997) Homotypic vacuolar fusion mediated by $\mathrm{t}-$ and v-SNAREs. Nature, 387, 199-202.

Novick, P., Ferro, S. and Schekman, R. (1981) Order of events in the yeast secretory pathway. Cell, 25, 461-469.

Novick, P., Field, C. and Schekman, R. (1980) Identification of 23 complementation groups required for post-translational events in the yeast secretory pathway. Cell, 21, 205-215.

Oakley, M.G. and Kim, P.S. (1998) A buried polar interaction can direct the relative orientation of helices in a coiled coil. Biochemistry, 37, 1260312610.

Ossig, R., Schmitt, H.D., de Groot, B., Riedel, D., Keranen, S., Ronne, H., Grubmuller, H. and Jahn, R. (2000) Exocytosis requires asymmetry in the central layer of the SNARE complex. Embo J, 19, 6000-6010. 
Otto, H., Hanson, P.I. and Jahn, R. (1997) Assembly and disassembly of a ternary complex of synaptobrevin, syntaxin, and SNAP-25 in the membrane of synaptic vesicles. Proc Natl Acad Sci U S A, 94, 61976201.

Palade, G. (1975) Intracellular aspects of the process of protein synthesis. Science, 189, 347-358.

Parlati, F., McNew, J.A., Fukuda, R., Miller, R., Sollner, T.H. and Rothman, J.E. (2000) Topological restriction of SNARE-dependent membrane fusion. Nature, 407, 194-198.

Paumet, F., Brugger, B., Parlati, F., McNew, J.A., Sollner, T.H. and Rothman, J.E. (2001) A t-SNARE of the endocytic pathway must be activated for fusion. J Cell Biol, 155, 961-968.

Paumet, F., Rahimian, V. and Rothman, J.E. (2004) The specificity of SNAREdependent fusion is encoded in the SNARE motif. Proc Natl Acad Sci $U$ $S$ A, 101, 3376-3380.

Pelham, H.R. (2001) SNAREs and the specificity of membrane fusion. Trends Cell Biol, 11, 99-101.

Peters, C., Andrews, P.D., Stark, M.J., Cesaro-Tadic, S., Glatz, A., Podtelejnikov, A., Mann, M. and Mayer, A. (1999) Control of the terminal step of intracellular membrane fusion by protein phosphatase 1. Science, 285, 1084-1087.

Peters, C., Bayer, M.J., Buhler, S., Andersen, J.S., Mann, M. and Mayer, A. (2001) Trans-complex formation by proteolipid channels in the terminal phase of membrane fusion. Nature, 409, 581-588.

Peters, C. and Mayer, A. (1998) Ca2+/calmodulin signals the completion of docking and triggers a late step of vacuole fusion. Nature, 396, 575-580.

Pfeffer, S.R. (1999) Transport-vesicle targeting: tethers before SNAREs. Nat Cell Biol, 1, E17-22.

Pobbati, A.V., Razeto, A., Boddener, M., Becker, S. and Fasshauer, D. (2004) Structural basis for the inhibitory role of tomosyn in exocytosis. J Biol Chem, 279, 47192-47200.

Poirier, M.A., Xiao, W., Macosko, J.C., Chan, C., Shin, Y.K. and Bennett, M.K. (1998) The synaptic SNARE complex is a parallel four-stranded helical bundle. Nat Struct Biol, 5, 765-769.

Prekeris, R., Klumperman, J., Chen, Y.A. and Scheller, R.H. (1998) Syntaxin 13 mediates cycling of plasma membrane proteins via tubulovesicular recycling endosomes. J Cell Biol, 143, 957-971.

Prekeris, R., Yang, B., Oorschot, V., Klumperman, J. and Scheller, R.H. (1999) Differential roles of syntaxin 7 and syntaxin 8 in endosomal trafficking. Mol Biol Cell, 10, 3891-3908.

Price, A., Seals, D., Wickner, W. and Ungermann, C. (2000) The docking stage of yeast vacuole fusion requires the transfer of proteins from a cisSNARE complex to a Rab/Ypt protein. J Cell Biol, 148, 1231-1238.

Rieder, S.E. and Emr, S.D. (1997) A novel RING finger protein complex essential for a late step in protein transport to the yeast vacuole. Mol Biol Cell, 8, 2307-2327.

Robinson, L.J., Aniento, F. and Gruenberg, J. (1997) NSF is required for transport from early to late endosomes. J Cell Sci, 110 ( Pt 17), 20792087.

Rothman, J.E. (1994) Intracellular membrane fusion. Adv Second Messenger Phosphoprotein Res, 29, 81-96. 
Rothman, J.E. and Warren, G. (1994) Implications of the SNARE hypothesis for intracellular membrane topology and dynamics. Curr Biol, 4, 220-233.

Rubino, M., Miaczynska, M., Lippe, R. and Zerial, M. (2000) Selective membrane recruitment of EEA1 suggests a role in directional transport of clathrin-coated vesicles to early endosomes. J Biol Chem, 275, 37453748.

Sambrook, J.a.R., D.W. (2001) Molecular Cloning: a laboratory manual. 3rd edition.

Sato, T.K., Rehling, P., Peterson, M.R. and Emr, S.D. (2000) Class C Vps protein complex regulates vacuolar SNARE pairing and is required for vesicle docking/fusion. Mol Cell, 6, 661-671.

Schagger, H. and von Jagow, G. (1987) Tricine-sodium dodecyl sulfatepolyacrylamide gel electrophoresis for the separation of proteins in the range from 1 to $100 \mathrm{kDa}$. Anal Biochem, 166, 368-379.

Schuette, C.G., Hatsuzawa, K., Margittai, M., Stein, A., Riedel, D., Kuster, P., Konig, M., Seidel, C. and Jahn, R. (2004) Determinants of liposome fusion mediated by synaptic SNARE proteins. Proc Natl Acad Sci U S A, 101, 2858-2863.

Seron, K., Tieaho, V., Prescianotto-Baschong, C., Aust, T., Blondel, M.O., Guillaud, P., Devilliers, G., Rossanese, O.W., Glick, B.S., Riezman, H., Keranen, S. and Haguenauer-Tsapis, R. (1998) A yeast t-SNARE involved in endocytosis. Mol Biol Cell, 9, 2873-2889.

Sesaki, H. and Jensen, R.E. (2001) UGO1 encodes an outer membrane protein required for mitochondrial fusion. J Cell Biol, 152, 1123-1134.

Simonsen, A., Gaullier, J.M., D'Arrigo, A. and Stenmark, H. (1999) The Rab5 effector EEA1 interacts directly with syntaxin-6. J Biol Chem, 274, 2885728860.

Simonsen, A., Lippe, R., Christoforidis, S., Gaullier, J.M., Brech, A., Callaghan, J., Toh, B.H., Murphy, C., Zerial, M. and Stenmark, H. (1998) EEA1 links $\mathrm{PI}(3) \mathrm{K}$ function to Rab5 regulation of endosome fusion. Nature, 394, 494498.

Sollner, T., Whiteheart, S.W., Brunner, M., Erdjument-Bromage, H., Geromanos, S., Tempst, P. and Rothman, J.E. (1993) SNAP receptors implicated in vesicle targeting and fusion. Nature, 362, 318-324.

Sorensen, J.B., Nagy, G., Varoqueaux, F., Nehring, R.B., Brose, N., Wilson, M.C. and Neher, E. (2003) Differential control of the releasable vesicle pools by SNAP-25 splice variants and SNAP-23. Cell, 114, 75-86.

Starai, V.J., Thorngren, N., Fratti, R.A. and Wickner, W. (2005) lon regulation of homotypic vacuole fusion in Saccharomyces cerevisiae. J Biol Chem, 280, 16754-16762.

Steegmaier, M., Klumperman, J., Foletti, D.L., Yoo, J.S. and Scheller, R.H. (1999) Vesicle-associated membrane protein 4 is implicated in transGolgi network vesicle trafficking. Mol Biol Cell, 10, 1957-1972.

Stenmark, H., Aasland, R. and Driscoll, P.C. (2002) The phosphatidylinositol 3phosphate-binding FYVE finger. FEBS Lett, 513, 77-84.

Stenmark, H., Vitale, G., Ullrich, O. and Zerial, M. (1995) Rabaptin-5 is a direct effector of the small GTPase Rab5 in endocytic membrane fusion. Cell, 83, 423-432.

Struck, D.K., Hoekstra, D. and Pagano, R.E. (1981) Use of resonance energy transfer to monitor membrane fusion. Biochemistry, 20, 4093-4099. 
Subramaniam, V.N., Loh, E., Horstmann, H., Habermann, A., Xu, Y., Coe, J., Griffiths, G. and Hong, W. (2000) Preferential association of syntaxin 8 with the early endosome. J Cell Sci, 113 ( Pt 6), 997-1008.

Sudhof, T.C. (2004) The synaptic vesicle cycle. Annu Rev Neurosci, 27, 509547.

Sun, W., Yan, Q., Vida, T.A. and Bean, A.J. (2003) Hrs regulates early endosome fusion by inhibiting formation of an endosomal SNARE complex. J Cell Biol, 162, 125-137.

Sutton, R.B., Fasshauer, D., Jahn, R. and Brunger, A.T. (1998) Crystal structure of a SNARE complex involved in synaptic exocytosis at $2.4 \mathrm{~A}$ resolution. Nature, 395, 347-353.

Takai, Y., Sasaki, T. and Matozaki, T. (2001) Small GTP-binding proteins. Physiol Rev, 81, 153-208.

Takei, K., Mundigl, O., Daniell, L. and De Camilli, P. (1996) The synaptic vesicle cycle: a single vesicle budding step involving clathrin and dynamin. $J$ Cell Biol, 133, 1237-1250.

Tang, B.L., Low, D.Y., Tan, A.E. and Hong, W. (1998a) Syntaxin 10: a member of the syntaxin family localized to the trans-Golgi network. Biochem Biophys Res Commun, 242, 345-350.

Tang, B.L., Tan, A.E., Lim, L.K., Lee, S.S., Low, D.Y. and Hong, W. (1998b) Syntaxin 12, a member of the syntaxin family localized to the endosome. J Biol Chem, 273, 6944-6950.

Thorngren, N., Collins, K.M., Fratti, R.A., Wickner, W. and Merz, A.J. (2004) A soluble SNARE drives rapid docking, bypassing ATP and Sec17/18p for vacuole fusion. Embo J, 23, 2765-2776.

Titorenko, V.I. and Rachubinski, R.A. (2000) Peroxisomal membrane fusion requires two AAA family ATPases, Pex1p and Pex6p. J Cell Biol, 150, 881-886.

Ungermann, C., Nichols, B.J., Pelham, H.R. and Wickner, W. (1998) A vacuolar v-t-SNARE complex, the predominant form in vivo and on isolated vacuoles, is disassembled and activated for docking and fusion. $J$ Cell Biol, 140, 61-69.

Ungermann, C., Price, A. and Wickner, W. (2000) A new role for a SNARE protein as a regulator of the Ypt7/Rab-dependent stage of docking. Proc Natl Acad Sci U S A, 97, 8889-8891.

Ungermann, C., Wickner, W. and Xu, Z. (1999) Vacuole acidification is required for trans-SNARE pairing, LMA1 release, and homotypic fusion. Proc Natl Acad Sci U S A, 96, 11194-11199.

Valdez, A.C., Cabaniols, J.P., Brown, M.J. and Roche, P.A. (1999) Syntaxin 11 is associated with SNAP-23 on late endosomes and the trans-Golgi network. J Cell Sci, 112 ( Pt 6), 845-854.

van der Bliek, A.M. and Meyerowitz, E.M. (1991) Dynamin-like protein encoded by the Drosophila shibire gene associated with vesicular traffic. Nature, 351, 411-414.

Voets, T., Toonen, R.F., Brian, E.C., de Wit, H., Moser, T., Rettig, J., Sudhof, T.C., Neher, E. and Verhage, M. (2001) Munc18-1 promotes large dense-core vesicle docking. Neuron, 31, 581-591.

Vogel, K. and Roche, P.A. (1999) SNAP-23 and SNAP-25 are palmitoylated in vivo. Biochem Biophys Res Commun, 258, 407-410. 
Wang, L., Merz, A.J., Collins, K.M. and Wickner, W. (2003) Hierarchy of protein assembly at the vertex ring domain for yeast vacuole docking and fusion. J Cell Biol, 160, 365-374.

Wang, L., Seeley, E.S., Wickner, W. and Merz, A.J. (2002) Vacuole fusion at a ring of vertex docking sites leaves membrane fragments within the organelle. Cell, 108, 357-369.

Ward, D.M., Leslie, J.D. and Kaplan, J. (1997) Homotypic lysosome fusion in macrophages: analysis using an in vitro assay. J Cell Biol, 139, 665-673.

Waters, M.G., Clary, D.O. and Rothman, J.E. (1992) A novel 115-kD peripheral membrane protein is required for intercisternal transport in the Golgi stack. J Cell Biol, 118, 1015-1026.

Weber, T., Zemelman, B.V., McNew, J.A., Westermann, B., Gmachl, M., Parlati, F., Sollner, T.H. and Rothman, J.E. (1998) SNAREpins: minimal machinery for membrane fusion. Cell, 92, 759-772.

Wen, J., Arakawa, T. and Philo, J.S. (1996) Size-exclusion chromatography with on-line light-scattering, absorbance, and refractive index detectors for studying proteins and their interactions. Anal Biochem, 240, 155-166.

Whiteheart, S.W., Griff, I.C., Brunner, M., Clary, D.O., Mayer, T., Buhrow, S.A. and Rothman, J.E. (1993) SNAP family of NSF attachment proteins includes a brain-specific isoform. Nature, 362, 353-355.

Wickner, W. (2002) Yeast vacuoles and membrane fusion pathways. Embo J, 21, 1241-1247.

Wilson, D.W., Wilcox, C.A., Flynn, G.C., Chen, E., Kuang, W.J., Henzel, W.J., Block, M.R., Ullrich, A. and Rothman, J.E. (1989) A fusion protein required for vesicle-mediated transport in both mammalian cells and yeast. Nature, 339, 355-359.

Woodman, P.G. and Warren, G. (1991) Isolation of functional, coated, endocytic vesicles. J Cell Biol, 112, 1133-1141.

Wu, M.N., Littleton, J.T., Bhat, M.A., Prokop, A. and Bellen, H.J. (1998) ROP, the Drosophila Sec1 homolog, interacts with syntaxin and regulates neurotransmitter release in a dosage-dependent manner. Embo J, 17, 127-139.

Wurmser, A.E., Sato, T.K. and Emr, S.D. (2000) New component of the vacuolar class $\mathrm{C}-\mathrm{V}$ ps complex couples nucleotide exchange on the Ypt7 GTPase to SNARE-dependent docking and fusion. J Cell Biol, 151, 551562.

Xu, Y., Wong, S.H., Tang, B.L., Subramaniam, V.N., Zhang, T. and Hong, W. (1998) A 29-kilodalton Golgi soluble N-ethylmaleimide-sensitive factor attachment protein receptor (Vti1-rp2) implicated in protein trafficking in the secretory pathway. J Biol Chem, 273, 21783-21789.

Yang, B., Gonzalez, L., Jr., Prekeris, R., Steegmaier, M., Advani, R.J. and Scheller, R.H. (1999) SNARE interactions are not selective. Implications for membrane fusion specificity. J Biol Chem, 274, 5649-5653.

Zerial, M. and McBride, H. (2001) Rab proteins as membrane organizers. Nat Rev Mol Cell Biol, 2, 107-117.

Zwilling Master Thesis, D. (2001) Characterization of endosomal SNAREs by application of a liposomal fusion assay. Georg August University, Göttingen. 


\section{Publications}

Assignment of the beta- $\mathrm{N}$-acetylhexosaminidase gene (HEXB) to porcine chromosome SSC2q21-->q22 by fluorescence in situ hybridisation and by analysis of somatic cell and radiation hybrid panels. Mueller A, Knorr C, Habermann F, Slanchev K, Zwilling D, Fries R, Brenig B. Institute of Veterinary Medicine, Georg August University of Göttingen, Germany

Cytogenetic and Genome Research, 2003;101(2):178.

SNAREs do not encode for the specificity of early endosomal homotypic fusion

Dorothea Brandhorst", Daniel Zwilling*, Silvio O. Rizzoli", Thorsten Lang, and Reinhard Jahn. *these authors contributed equally to the work

Department of Neurobiology, Max-Planck-Institute for Biophysical Chemistry, 37077 Göttingen, Germany

submitted

Evidence for the involvement of endosomal fusion in synaptic vesicle recycling

Silvio O. Rizzoli, Ioanna Bethani*, Daniel Zwilling*, Dirk Wenzel, Dorothea Brandhorst and Reinhard Jahn. *these authors contributed equally to the work

Department of Neurobiology, Max-Planck-Institute for Biophysical Chemistry, 37077 Göttingen, Germany

submitted 


\section{Curriculum Vitae}

\section{Personal Information}

Name:

Daniel Hans-Georg Heinrich Zwilling

Date of birth:

16.03.1974

Place of birth:

Frankfurt, Germany

Address:

Burg Str. 37/A, Göttingen, 37073 Germany

\section{University}

2002-Present: Ph.D. in Molecular Biology, Int. Max Planck Research School Göttingen (IMPRS)

Department of Neurobiology (Prof. Jahn), Max-Planck-Institute for biophysical chemistry, Göttingen

Titel of Thesis: Characterisation of the early endosomal SNARE complex by application of a liposomal fusion assay

2000-2002: $\quad$ M.Sc. in Molecular Biology International M.Sc./Ph.D Programme in Biology Int. Max Planck Research School, Göttingen

1999-2000: $\quad$ Upper division Biology, Georg-August-Universität 1997-1998: $\quad$ Education Abroad Program at University of California Los Angeles, MCDB (Molecular Cell and Developmental Biology) division

1995-1997: $\quad$ Undergraduate studies in Biology, Georg-AugustUniversität, Göttingen

\section{Civilian Service}

1994-1995: $\quad$ Selbsthilfe Körperbehindeter e.V., Göttingen

\section{Primary and secondary education}

1989-1993: $\quad$ Ziehen Schule, Frankfurt

1984-1989: Humboldt Schule, Bad Homburg

1983-1984: $\quad$ Fritz Husmann Schule, Bremerhaven

1980-1983: $\quad$ Adolf Reichwein Schule, Neu-Anspach 


\section{Acknowledgements}

I would like to thank Prof. Reinhard Jahn for his support and help and for giving me the opportunity to work in his laboratory. Also, I want to thank my two cosupervisors Prof. Nils Brose and Prof. Kurt von Figura for their support and helpful criticism.

I would like to thank Dr. Steffen Burkhardt and Nina McGuinness for their help and support, for providing a great atmosphere for all students of the International Max-Planck research school in Göttingen and for always being there if help was needed. Also, I want to thank all the students who accompanied me in the master's year.

I am very thankful to my colleagues in the Neurobiology department to provide a relaxed but very professional working atmosphere and for the help they are always providing. I want to express thanks to Henning Urlaub and Uwe Plessmann for the many gels they analysed for me and Markus Wahl for the help in crystallising and providing me with the crystal data analysis. I am very grateful to Ursel Ries for helping me with protein expressions and purification, Maria Druminski for help with cloning and Gottfried Mieskes for always providing help and advice whenever it was needed. I want to thank Dirk Fasshauer for discussions and suggestions that improved my experimental work and Silvio Rizzoli, Thorsten Lang, Matthew Holt, Pawel Burkhardt and Prof. Sölling who gave me helpful suggestions when I wrote this thesis.

I am grateful to my parents and my family and friends in Germany and in Trinidad for their love and support.

I thank my beloved wife Yaisa for her love, for always being there for me, for introducing me to the Trini-culture, for sharing her time, friendship and humour with me and making my live wonderful. 